\title{
Investigating the effects of novel heparan sulfate mimetic 'HSI6-35' on immune cell migration in an in vitro model of the blood-CSF barrier.
}

\author{
By Madeline Georgia Griffiths
}

\author{
A thesis submitted to \\ Victoria University of Wellington \\ In fulfilment of the requirements for the degree of \\ Master of Biomedical Science
}

Victoria University of Wellington

Te Kura Mātauranga Koiora 

This thesis was conducted under the supervision of

\section{Prof. Anne La Flamme}

Victoria University of Wellington

and

Malaghan Institute of Medical Research

Wellington, New Zealand 

This thesis is dedicated in full to the late Annette Fairweather (1928-20/4)

If you only knew how often I thank you. 



\section{ABSTRACT}

The central nervous system was traditionally considered an immune-privileged site, defined as being immunologically inactive. However, recent studies have elucidated that a number of immune cells traffic into and out of the brain in healthy humans to conduct routine immunosurveillance. $A$ unique immunological interface, the choroid plexus, acts as a gatekeeper for the entry of these immune cells during homeostasis. Although the mechanisms are not well described, the choroid plexus also has the capacity to regulate the responses of migrating leukocytes during inflammation.

Multiple sclerosis is a complex neuroinflammatory disease characterized by demyelination in the CNS. Autoreactive immune cells invade the central nervous system and orchestrate an attack against myelin sheathes, the insulation layer that protects neurons. The disease affects nearly I in I,000 New Zealanders, and currently has no cure. The most successful treatments for multiple sclerosis target the initial stages by inhibiting the entry of these cells into the central nervous system, however these are often associated with severe side and life-threatening effects and cannot prevent the progression of the disease.

Heparanase, the ubiquitously expresses heparan sulfate degrading enzyme has been thoroughly implicated in the disease processes of multiple sclerosis, and its animal model, EAE. Autoreactive lymphocytes exploit heparanase activity to degrade the extracellular matrix and destabilize the barriers that maintain the relative immune privileged status of the central nervous system. Exogenous heparan sulfate mimetics have previously been shown to ameliorate symptoms of EAE by interfering with heparanase activity. However, the commercialization and clinical translation of these inhibitors is currently inhibited by the complexity of their synthesis. 'HSI6-35' is a novel heparan sulfate mimetic developed by the Ferrier Institute, comprised of a dendritic core with four heavily sulfated oligosaccharide arms. The synthesis of this compound is much shorter due to its smaller size; however, it has been shown to act similarly to native heparan sulfate molecules. We proposed that $\mathrm{HSI6-35}$ is protective in preventing the migration of autoreactive immune cells across the choroid plexus by inhibiting lymphocyte heparanase.

To investigate the efficacy of HSI6-35 in vitro, we first established an experimental transwell model of the choroid plexus. This model incorporated core components of the choroid plexus, including fenestrated capillaries, the stromal matrix and epithelial monolayer. We first showed that the model was capable of mimicking homeostatic trafficking across the choroid plexus epithelium, which formed a selective but permeable barrier. Then, we induced T-cell specific inflammatory migration using Concanavalin $A$ or $T_{H} I-$ 
type cytokines. This migration was found to be interferon- $\gamma$ dependent and could be mitigated with antiinterferon- $\gamma$ treatment.

Once this model was established, we next investigated whether HSI6-35 was effective in inhibiting inflammatory migration across this structure. To adapt HSI6-35 to an in vitro dose, we performed cell viability assays. This confirmed that the compound was mildly cytotoxic to epithelial choroid plexus cells but not murine splenocytes. Further experiments found that low-dose HSI6-35 did not impact monolayer permeability. Transwell migration assays showed that low-dose HSI6-35 was effective in reducing ConA and interferon- $\gamma$ mediated inflammatory T-cell migration to a level comparable to homeostatic trafficking. Finally, we assessed cytokine profiles of leukocytes and epithelial choroid plexus cells treated with HSI6-35 and found that HSI6-35 reduced the expression of key cytokines involved in MS pathogenesis.

In summary, the work described in this thesis showed how HSI6-35 may be protective during EAE by suppressing the inflammatory response of autoreactive T-cells, in addition to regulating the infiltration of immune cells into the CNS through the choroid plexus. In a broader sense, these findings show that HSI6-36 may be effective in treating MS by regulating, not inhibiting lymphocyte migration into the CNS, mitigating some of the severe side effects that other migration-inhibitors face. 


\section{ACKNOWLEDGEMENTS}

First and foremost, l'd like to thank my wonderful supervisor Anne La Flamme for her endless support during this thesis. I am so incredibly honoured to have completed this degree under the supervision of such a powerwoman. Thank you for giving me the freedom to investigate the avenues that I found interesting, and for helping me put all the pieces back together at the end to tell (in my opinion, a pretty cool) bigger story. I'm so excited to continue this project into a doctorate degree with her supervision.

I'd also like to thank the OG LAF lab group; Afnan, Katharina, Lisa, Tessa, and Sven as well as our new additions; Mackenzie, Hasanah, Elysha, Connor and Blake. Those hours we spent at Wishbone are truly invaluable and I love you all. I could not have asked for a better lab group. Thank you for letting me bounce ideas off of you, and for letting me laugh and cry when I made dumb mistakes. A special thank you to Afnan and Katharina for their instrumental help in getting the Transwell migration model up and working, you both spent countless hours helping me with George when he was being a nightmare, mulling over results with me and finding ways to improve the experimental setup. I couldn't have done this without your help.

I think a second special thank you also needs to go to Afnan Al Abadey. From day one in this lab group, we clicked and I'm so grateful to have found a true bestie throughout the course of this thesis. Thank you for staying with me in the lab during late nights, the rides home, the fun we had on those skateboards and of course the deep life chats on oriental parade in the middle of the night. Special mention to that I2am call to the BD guy when you came to rescue me and kept your cool even though George was losing the plot. There are really no words to express the extent of how grateful I am, but I feel like l've found a sister in you. I love you so much and I'm looking forward to the next three years of doofy adventure with you during our PhD's.

I'd also like to thank my family, especially my wonderful parents, Karen and Torin, my brother Hadleigh and my lovely grandparents Robyn and Lindsay. Thank you, Mum, for coming down monthly to visit me and make sure I was eating properly. The time we spent together was invaluable. A special thank you also to Mum for helping me passage my cells in TC those few times. That was instrumental to the success of this thesis. I also treasure the trips I had back to Taradale and all the fishing that Dad, Grandad, and I got to do this year. Those moments of peace truly kept me sane. Thank you, Dad, for staying up and chatting with me whilst we watched the MotoGP together in the middle of the night. A huge thank you to my 
beautiful Grandmother, Robyn who has been the number one supporter of this thesis and got me through a lot of my imposter syndrome. You and Grandad have never faltered in your support for me and I wish you all the blessings in the world. Hadz, thank you for being there to support me, tagging me in silly Facebook memes and having some good chats throughout it all. Thank you to my dogs, Louie and Georgie for your endless love and snuggles.

l'd like to thank the wonderful Fabian, for supporting me throughout the completion of this thesis. If not for you, l'd never have pursued graduate school, and I am endlessly grateful that you pushed me in that direction. Thank you for setting me up with Anne all the way back in second year. Because of that, I have had so many opportunities to develop in my research career and as a person. Thank you for talking me down from where I felt like the world was ending and providing all the technical support for this thesis. Your compassion and kindness in the midst of it all was so welcomed and I hope you know how grateful I am.

Finally, l'd like to thank all the funding bodies that drove this research, namely The Great New Zealand Trek. To all those who participated and donated, I can never express my gratitude enough. Thank you. Thank you. Thank you. 


\section{CONTENTS}

\section{ABSTRACT}

I.I.I. INNATE IMMUNITY

I.I.2. ADAPTIVE IMMUNITY

I.2. B CELLS

I.3. T CELLS 16

I.3.1. CD8+ T CELLS

I.3.2. CD4+ T CELLS

1.3.3. REgUlatory T CELLS

I.4. Central Nervous System Immune Privilege 20

I.5. Multiple SClerosis 22

I.5.I. RISK FACTORS 22

1.5.2. IMMUNOPATHOLOGY 23

1.5.3. TREATMENTS 24

I.6. EAE AS A MODEL OF MS 28

I.7. The ChOROID PleXUS 29

I.8. Heparanase/heparan SUlfate 32

I.9. HSI6-35 35

I. 10. RESEARCH AIMS 
$\begin{array}{lr}\text { 2.I. Materials } & 40\end{array}$

$\begin{array}{lll}\text { 2.I.I. TABLE OF MATERIALS } & 40\end{array}$

2.2. Animals and Tissue Harvest

2.2.1. ANIMALS 4I

2.2.2. ETHICS DECLARATION 4I

2.2.3. EAE INDUCTION IN ANIMALS

2.2.4. TISSUE ISOLATION

$\begin{array}{ll}\text { 2.2.5. SPLENOCYTE ISOLATION } & 42\end{array}$

$\begin{array}{ll}\text { 2.2.6. SPLENOCYTE VIABILITY ASSESSMENT } & 42\end{array}$

2.3. General Epithelial Cell Culture 43

$\begin{array}{lll}\text { 2.3.I. ECPC-4 CELL CULTURE } & 43\end{array}$

2.3.2. ThaWING CelLS

2.3.3. Passaging Cells 43

$\begin{array}{lll}\text { 2.3.4. } & \text { FreeZING CellS } & 44\end{array}$

2.4. establishing a Transwell Monolayer Culture 45

2.4.I. Preparation of Transwells for Cell Culture 45

2.4.2. SPLENOCYTE StimULATION 46

2.4.3. Treatment Of the Transwell Culture With HSI5-35 46

2.5. Transwell Migration Assay 48

$\begin{array}{lll}\text { 2.5.I. CYTOKINE STIMULATION: IFN } r & 48\end{array}$

$\begin{array}{lll}\text { 2.5.2. SPLENOCYTE MIGRATION } & 48\end{array}$

2.5.3. CHEMOKINE StIMULATION: CCL5 49

2.6. FLOW CYTOMETRY

$\begin{array}{lll}\text { 2.6.I. ANTIBODY LIST } & 50\end{array}$

$\begin{array}{ll}\text { 2.6.2. PREPARATION OF SOlVENTS } & 50\end{array}$

2.6.3. FULl PANel STAIN 5 I

2.6.4. Flow Cytometry Protocol 5I

$\begin{array}{ll}\text { 2.7. IFNr ELISA } & 53\end{array}$

$\begin{array}{lll}\text { 2.7.I. STANDARDS } & 53\end{array}$ 
2.7.2. ELISA PROTOCOL 53

$\begin{array}{ll}\text { 2.8. MTT ASSAY } & 54\end{array}$

2.8.I. CeLL SPECIMENS $\quad 54$

2.9. Evans Blue Permeability Assay 56

2.9.1. SOLVENTS $\quad 56$

2.9.2. PermeAbility Assay Protocol $\quad 56$

2.10. LEGENDPLEX ${ }^{\mathrm{TM}}$ MOUSE INFLAMMATION PANEL $\quad 58$

2.10.1. Preparation OF Tissue Culture Supernatant 58

$\begin{array}{ll}\text { 2.10.2. STANDARD PREPARATION } & 58\end{array}$

2.10.3. ASSAY PROCEDURE $\quad 58$

$\begin{array}{lr}\text { 2.II. StAtistics } & 59\end{array}$

$\begin{array}{ll}\text { SUPPLEMENTARY METHODS } & 6 \text { I }\end{array}$

2.12. establishing a Transwell Monolayer Culture 6 I

2.12.I. Preparation of Transwells for Cell Culture 6 I

2.12.2. TRANSWELl Migration AsSAY 61

2.13. MTT ASSAY: CCL5 TOXICITY 63

2.13.I. CELL SPECIMENS $\quad 63$

2.13.2. TREATMENT CONDITIONS 63

2.13.3. MTT ASSAY PROTOCOL 63

2.14. REAGENT FORMULAS $\quad 64$

2.14.I. Complete Growth Media (CGM) 64

2.14.2. Complete TisSue Culture Media (CTCM) 64

2.14.3. WASH BUFFER $\quad 64$

2.I4.4. DULBECCO’S PHOSPHATE-BUfFERED SALINE

2.14.5. FluOresCence-Activated Cell SORTING (FACS) Buffer 65

$\begin{array}{lll}\text { 2.14.6. IOX PHOSPHATE-BufFERED SALINE } & 65\end{array}$

$\begin{array}{lll}\text { 2.14.7. ELISA BUFFERS } & 65\end{array}$

2.14.8. MTT BUFFERS $\quad 65$

$\begin{array}{lll}\text { 2.14.9. PERTUSSIS TOXIN BUfFER } & 65\end{array}$ 
CHAPTER 3: DEVELOPING AN IN VITRO MODEL OF THE CHOROID PLEXUS FOR

$\begin{array}{ll}\text { 3.I. INTRODUCTION } & 68\end{array}$

$\begin{array}{lr}\text { 3.2. AIMS } & 69\end{array}$

$\begin{array}{llr}\text { 3.2.I. SPECIFIC AIMS } & 69\end{array}$

$\begin{array}{ll}\text { 3.3. Results } & \mathbf{7 0}\end{array}$

3.3.I. CCL5 IS A NON-TOXIC CHEMOKINE TO SPLENOCYTES

3.3.2. MATRIGEL IS NOT A SUITABLE StROMAL MATRIX COMPONENT 72

3.3.3. ECPC-4 CELLS ARE CAPABLE OF FORMING A FUNCTIONAL MONOLAYER 75

3.3.4. INFLAMMATORY MIGRATION CAN BE INDUCED WITH CONCANAVALIN A STIMULATION 77

3.3.5. INFLAMMATORY MIGRATION INDUCED WITH CONA IS PARTIALLY IFN I DEPENDENT 80

$\begin{array}{ll}\text { 3.4. DisCUSSION } & 82\end{array}$

$\begin{array}{ll}\text { 3.5. SUMMARY } & 88\end{array}$

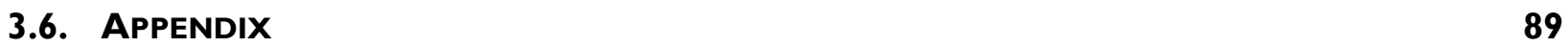

CHAPTER 4: DOES THE HEPARAN SULFATE MIMETIC ‘HSI 6 35’ AFFECT

LEUKOCYTE MIGRATION IN AN EXPERIMENTAL MODEL OF THE CHOROID

PLEXUS?

$\begin{array}{ll}\text { 4.I. INTRODUCTION } & 92\end{array}$

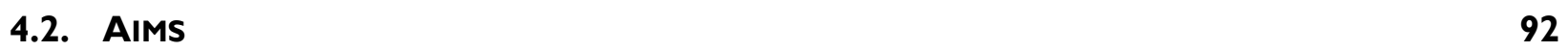

4.2.I. SPECIFIC AIMS $\quad 92$

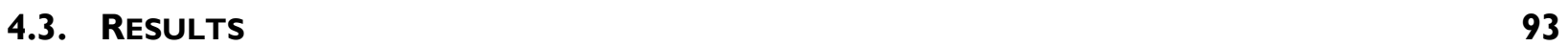

4.3.I. HSI6-35 IS NON-TOXIC TO MURINE SPLENOCYTES, BUT EXERTS MILDLY CYTOTOXIC EFFECTS ON $\begin{array}{ll}\text { ECPC-4 CELLS } & 93\end{array}$

4.3.2. HSI6-35 INHIBITS MIGRATION IN A TRANSWELL MODEL OF THE CHOROID PLEXUS 96

4.3.3. HSI6-35 MAY BE EFFECTIVE AS AN ANTIMIGRATORY AGENT BY INHIBITING PROINFLAMMATORY CYTOKINE RELEASE

4.3.4. HSI6-35 MAY BE EFFECTIVE IN INHIBITING ENCEPHALITOGENIC LEUKOCYTE ENTRY THROUGH A $\begin{array}{ll}\text { MODEL OF THE CHP } & 116\end{array}$

4.4. Discussion 



\section{LIST OF FIGURES}

FIGURE I.I: THE COMPOSITION OF INTERCELLULAR MOLECULES THAT MAINTAIN THE BLOOD-CSF BARRIER

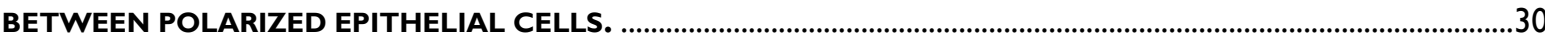

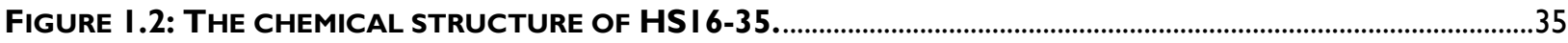

FIgURE 2. I: SIMPLIFIED SCHEMATIC SHOWING HOW WE SEED ECPC-3 CELLS ONTO A TRANSWELL.............45

FIGURE 2.2: SIMPLIFIED SCHEMATIC SHOWING HOW THE TRANSWELL MIGRATION ASSAY IS RUN...................48

FIGURE 2.3: SIMPLIFIED SCHEMATIC SHOWING HOW THE EBD PERMEABILITY ASSAY IS RUN. ..........................57

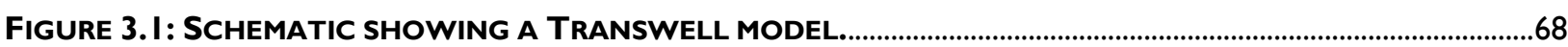

FIGURE 3.2: HIGH CONCENTRATIONS OF CCL5 DECREASE SPLENOCYTE VIABILITY.......................................... I

FIGURE 3.3: THE GATING STRATEGY USED TO ANALYSE FLOW CYTOMETRY DATA FROM THE MIGRATION EXPERIMENTS IN CHAPTER 3.

FIgURE 3.4: MATRIGEL IS NOT A SUITABLE BASEMENT MEMBRANE COMPONENT FOR STUDYING LEUKOCYTE TRAFFICKING.

FIGURE 3.5: ECPC-4 CELLS ARE CAPABLE OF FORMING AN INTACT BUT PERMEABLE BARRIER ON TRANSWELLS AFTER 24H.

FIGURE 3.6: CONA INDUCES INFLAMMATORY MIGRATION ACROSS THE ECPC-4 MONOLAYER. .78

FIGURE 3.7: CONA ELICITS A GREATER MIGRATORY RESPONSE FROM T CELLS. .79

FIGURE 3.8: CONA INDUCES IFNr DEPENDENT MIGRATION, WHICH CAN BE REDUCED WITH IFNr NEUTRALISING ANTIBODY.

FIGURE 3.9: SCHEMATIC DEMONSTRATING HOW CORE COMPONENTS OF THE BCSFB WERE ADAPTED TO AN IN VITRO MODEL.

FIGURE 3. I 0: CONA INDUCES INCREASED MIGRATION ACROSS OUR TRANSWELL MODEL: ................................89

FIGURE 3.I I: CONA INDUCES INCREASED MIGRATION ACROSS OUR TRANSWELL MODEL.................................90

FIGURE 4. I : HS I 6-35 IS MILDLY CYTOTOXIC TO ECPC-4 CELLS BUT DOES NOT INTERRUPT MONOLAYER PERMEABILITY.

FIGURE 4.2. HSI 6-35 DOES NOT EXERT CYTOTOXIC EFFECTS ON HEALTHY OR ENCEPHALITOGENIC SPLENOCYTES.

FIGURE 4.3: OUR GATING STRATEGY FOR FLOW CYTOMETRY IN CHAPTER 4.

FIGURE 4.4: HSI 6-35 INHIBITS CONA-MEDIATED MIGRATION OF CD45 ${ }^{+}$LEUKOCYTES ACROSS AN EPITHELIAL MONOLAYER. HSI 6-35 APPEARS TO BE MORE EFFECTIVE IN INHIBITING CD4+ T CELLS, CD8 $^{+} \mathrm{T}$ CELLS AND DC

FIGURE 4.5: CONA INDUCES AN INCREASE IN MIGRATION ACROSS A TRANSWELL CULTURE WHICH IS MITIGATED BY HS I 6-35. 
Figure 4.6: HSI6-35 INHIBITS MIGRATION OF ON CD4 ${ }^{+} T_{\text {EM }}, T_{C M}$ AND CD8 ${ }^{+} T_{N}, T_{E M}, T_{C M}$ BUT NOT CD4 ${ }^{+}$ $T_{N}$ SUBSETS.

FIGURE 4.7: CONA INCREASES THE TOTAL NUMBER OF EFFECTOR AND CENTRAL MEMORY CD4 ${ }^{+} \mathrm{T}_{\text {CELLS }}$ BUT DECREASES THE TOTAL NUMBER OF EFFECTOR AND CENTRAL MEMORY CD8 ${ }^{+} \mathrm{T}$ CELLS .

FIGURE 4.8: HS I 6-35 REDUCED GENERAL LEUKOCYTE MIGRATION THROUGH A TRANSWELL MODEL OF THE CHP.

FIGURE 4.9: HSI6-35 REDUCED IFNI-MEDIATED CD4+ AND CD8+ T CELL MIGRATION THROUGH A MODEL OF THE CHP

FIGURE 4. I0: HSI 6 REDUCED B CELL, MYELOID AND DENDRITIC CELL MIGRATION THROUGH A TRANSWELL MODEL OF THE CHP.

FIGURE 4.I I: HS I6-35 MITIGATES PROINFLAMMATORY CYTOKINE RESPONSES FROM ENCEPHALITOGENIC SPLENOCYTES.

FIGURE 4. I 2: ECPC-4 CULTURES RELEASE INCREASING CONCENTRATIONS OF IFNI AND CCL2 WHICH CORRELATES WITH TREATMENT CONCENTRATION OF HS I6-35. 
Chapter I: General Introduction 


\section{Abbreviations}

\begin{tabular}{|c|c|c|c|}
\hline $\mathrm{Ab}$ & antibody & HSPG & heparan sulfate proteoglycans \\
\hline AEC & animal ethics committee & ICAM-I & intercellular adhesion molecule-I \\
\hline $\mathrm{Ag}$ & antigen & IFN $\gamma$ & interferon gamma \\
\hline ANOVA & analysis of variance & ILC & innate-lymphoid cell \\
\hline APC & antigen presenting cell & IL-x & interleukin-x \\
\hline BBB & blood-brain barrier & JAM & junctional adhesion molecule \\
\hline BCSFB & blood-CSF barrier & $\mathrm{JCV}$ & John-Cunningham virus \\
\hline CCL- & chemokine (c-c motif) ligand & MI & classically activated macrophage \\
\hline CDP & confirmed disability progression & M2 & alternatively activated macrophage \\
\hline CDx & cluster of differentiation & MAG & myelin associated protein \\
\hline CGM & complete growth media & MBP & myelin basic protein \\
\hline $\mathrm{ChP}$ & choroid plexus & $\mathrm{MHC} \mathrm{I/II}$ & major histocompatibility complex I/II \\
\hline CNS & central nervous system & MOG & myelin oligodendrocyte protein \\
\hline ConA & concanavalin A & MS & multiple sclerosis \\
\hline CSF & cerebral spinal fluid & PBS & phosphate buffered saline \\
\hline СТCM & complete tissue culture media & PML & progressive multifocal leukoencephalopathy \\
\hline CXCL- & chemokine (c-x-c motif) ligand & PPMS & primary progressive MS \\
\hline $\mathrm{Da}$ & Daltons & PRR & pattern-recognition receptor \\
\hline DC & dendritic cell & RRMS & relapsing remitting $\mathrm{MS}$ \\
\hline DMEM & Dulbecco's modified eagle medium & SPMS & secondary progressive MS \\
\hline DMF & dimethyl fumarate & TCR & T cell receptor \\
\hline DMSO & dimethyl sulfoxide & TLR & toll-like receptor \\
\hline DMT & disease modifying therapy & TNF $\alpha$ & tumour necrosis factor alpha \\
\hline dPBS & Dulbecco's phosphate buffered saline & Treg & $\mathrm{T}$ regulatory cell \\
\hline EAE & $\begin{array}{l}\text { experimental autoimmune } \\
\text { encephalomyelitis }\end{array}$ & UVR & ultraviolet radiation \\
\hline EBV & Epstein Barr virus & VCAM-I & vascular cell adhesion protein-I \\
\hline ECPC & epithelial choroid plexus cell & VLA-4 & $\alpha 4 \beta I$ integrin \\
\hline EDSS & expanded disability status score & $x g$ & relative centrifugal force \\
\hline GWAS & genome wide association study & ZO-I & zona occludins protein-I \\
\hline HS & heparan sulfate & $\alpha \mathrm{IFN} \gamma$ & anti-interferon gamma \\
\hline
\end{tabular}




\section{I.I. The immune system}

To survive, we must be protected from a continual onslaught of organisms that are inhaled, swallowed, or inhabit the skin and mucosa. The immune system is the defence force of the body, a network of special organs, circulating effector cells and molecules that prevent serious infection causing harm to the host. The immune system has two arms, each defined by the speed and specificity of their reactions: the innate and adaptive immune responses. Both systems are populations of white blood cells called leukocytes, originating from the hemopoietic stem cells of the bone marrow that commit to different lineage fates. Mature leukocytes may reside in peripheral tissue, circulate in the blood or travel through the lymphatic system, depending on the cell type and inflammatory state of the body.

\section{I.I.I. Innate Immunity}

The innate immune system is recognizable by its rapid, non-specific response to foreign bodies. The innate system paves the way for the adaptive immune system by managing the infection in its early stages and providing essential information about the type and nature of the pathogen. The cells of the innate immune system include; phagocytes (neutrophils, macrophages, monocytes, or dendritic cells); granulocytes (eosinophils, basophils, or mast cells) or innate lymphocytes (natural killer lymphocytes or innate lymphoid cells). These cells populate most peripheral tissues, allowing them to respond within minutes to a potential threat. Cells of the innate immune system are unique in that they are capable of identifying a vast number of pathogens. The functionality of the innate immune response relies on pattern recognition receptors (PRR), which are capable of recognising a limited number of pathogen associated molecular patterns (PAMPs) at the site of infection. ${ }^{1-3}$ The four classes of pathogen; viruses, bacteria, fungi and parasites that share class-specific molecular signatures detectable by PRRs. ${ }^{1-3}$ Toll-like receptors (TLRs) are transmembrane proteins capable of recognising extracellular bacteria or bacteria internalised in vesicles during phagocytosis. Cytoplasmic PRR's, including NOD-like receptors (NLR's) recognise intracellular bacteria and mRNA from viral infections. ${ }^{1-3} \mathrm{~A}$ key feature of the innate immune system is priming the adaptive immune response. The innate immune response lacks specificity, so on its own cannot effectively control an infection. ${ }^{2}$ It must pass on information about the pathogen to the adaptive immune system, as the adaptive response cannot initiate a response against a pathogen without input from the innate system. ${ }^{4}$

\section{I.I.2. Adaptive Immunity}

The adaptive response is characterized by a much slower, but more specific response. A key feature of the adaptive immune response is its capacity to form immunological memory, which allows the host to 
respond with greater speed and efficacy if it encounters the same pathogen at a later time. The cells of the adaptive immune system are B lymphocytes ( $B$ cells) and $T$ lymphocytes ( $T$ cells). B cells and $T$ cells express distinct antigen receptors and perform vastly different roles in the body. As described, this response lags behind the innate immune response, however these cells have the advantage of antigenspecific recognition of invading pathogens. They complete the immune arsenal to rid the body of infection and form immunological memory to protect the host if they encounter the same pathogen again.

\section{I.2. B cells}

B cells mature in the bone marrow and are known primarily for the production of antibodies and antigen presentation to $T$ cells. ${ }^{2,4}$ The initial antibodies produced by a naive B cell are not secreted, rather inserted into the membrane, functioning as a B-cell receptor (BCR). ${ }^{5}$ Each $B$ cell produces a single, unique type of antibody, with specificity to only one antigen at its binding site as according to the clonal selection theory. ${ }^{5}$ The secondary lymphoid organs house naïv B cells in the body, where they receive a constant influx of antigen in the lymph. ${ }^{2}$ When a $B$ cell recognises antigen on the BCR, the receptor-antigen complex is internalised and digested to produce peptide fragments to present to $\mathrm{T}$ cells. ${ }^{6} \mathrm{~B}$ cell activation generally relies on 'help' from follicular helper $T$ cells $\left(T_{F H}\right)$ at the border of the $T$ and $B$-cell zones. ${ }^{6}$ When $B$ cells present antigen loaded onto major histocompatibility II complexes (MHC), $T_{F H}$ cells conjugate the CD40 ligand to CD40 on the B-cell surface, followed by the release of stimulatory chemokines interleukin-4 (IL4) and IL-2I which induces clonal expansion. 2,6 Activated B cells migrate back into the interfollicular regions in response to chemokine gradients where they continue to proliferate and exert their effects. The majority of effector B cells will differentiate into short-lived plasma cells, with the rest becoming memory $\mathrm{B}$ cells, which can expand rapidly again upon antigen rechallenge.2,5 Antibodies produced by effector B cells aid in the overall immune response through neutralization, opsonisation and complement activation. $^{2}$

\section{I.3. T cells}

Precursor $\mathrm{T}$ cells are derived from the bone marrow but migrate to the thymus where they develop further. ${ }^{2}$ Like $B$ cells, each mature $T$ cell expresses a T-cell receptor (TCR) with unique specificity for a singular antigen. ${ }^{2}$ Development of the TCR in the thymus leads to two classes of T cells, the majority expressing the $\alpha \beta$ T-cell receptor, and $<5 \%$ bearing the $\gamma \delta$ receptor. ${ }^{7}$ The so called 'uniqueness' of the TCR relies on recombination of specific genes in the $\beta$-chain, tested and paired against an invariant $\alpha-$ 
chain, which is subsequently rearranged to form a novel TCR. ${ }^{8}$ Newly formed T cells then undergo positive and negative selection to ensure that the cell can recognise the MHC complex, but with an acceptable affinity, as those that bind too strongly have to potential to develop autoimmunity. ${ }^{2,8}$ Positive selection determines the class of $\mathrm{T}$ cell; i.e. if they recognise $\mathrm{MHC} \mathrm{I}$, they become $\mathrm{CD} 8^{+} \mathrm{T}$ cells, and those that interact with MHC II will become CD4+ T cells. ${ }^{7,8}$ Mature T cells leave the thymus to circulate between the blood and lymphoid organs, scavenging their specific antigen. ${ }^{2}$ To initiate an immune response $\mathrm{T}$ cells must be presented with a peptide antigen on the MHC complex of an APC, which induces clonal expansion and the differentiation into effector T cells. ${ }^{2}$ These effector cells have specific weaponry that allow them to remove the pathogen from the body, and the ability to respond immediately to future infection with the same pathogen. ${ }^{4}$

\section{I.3.I. CD8+ T cells}

Naive T cells bearing the co-receptor 'CD8' differentiate into CD8+ cytotoxic lymphocytes based on their ability to recognize $\mathrm{MHC}$ class I. CD8 ${ }^{+} \mathrm{T}$ cells provide vital immune defence against cytosolic pathogens and tumour surveillance.2,9 These cells are most known for their 'killer function', in which infected target cells present fragmented antigen from endogenous pathogens on $\mathrm{MHCl}$, and patrolling $\mathrm{CD} 8^{+}$lymphocytes recognise and induce apoptosis in these cells. ${ }^{9}$ Activated $\mathrm{CD} 8^{+} \mathrm{T}$ cells harbour several mechanisms to kill infected cells: the production of cytokines such as IL-2, IFN $\gamma$ and TNF $\alpha$, the release of cytotoxic granules or Fas/FasL interactions through the caspase pathway., 20 These mechanisms are discreet in that they avoid non-specific damage to the surrounding tissue. However, because these effector functions are so destructive, CD8+ T cells can contribute to excessive amounts of damage when the immune response becomes dysregulated in diseases such as MS."I

\section{I.3.2. CD4+ T cells}

$\mathrm{CD}^{+} \mathrm{T}$ cells recognize exogenous peptide presented on the MHC class II complex, and like CD8+ $\mathrm{T}$ cells, require antigen presentation for activation..$^{2,12}$ In contrast to $\mathrm{CD} 8^{+}$cytotoxic lymphocytes, CD4+ $\mathrm{T}$ cells then enter further stages of differentiation into $T$ helper cells $\left(T_{H}\right)$ to direct the proper immune response against a specific antigen. ${ }^{12} \mathrm{CD} 4^{+} \mathrm{T}$ cells do not act directly on their target cells, rather orchestrating the effector functions of other immune cells. ${ }^{4}$ This allows the host to form a specialised and cooperative response to each class of pathogen, clearing the infection more efficiently. There are many defined CD4+ T helper cell subsets, but the major players are: $T_{H} I, T_{H} 2, T_{H} 17$ and regulatory $T$ cells (Treg). ${ }^{13}$ These 
subsets are distinct in their cytokine determinants, fate-specific transcription factors and the immune cells they target for recruitment.

$\mathrm{T}_{\mathrm{H}} \mathrm{I}$ cells differentiate in response to the fate-specifying cytokines IFN $\gamma$ and IL- 12 produced by APC's early in infection. ${ }^{14}$ These cells can be recognised by their expression of transcription factor T-bet, which is turned on in response to IFN $\gamma$ signalling through the JAK-STAT pathway. ${ }^{15}$ Differentiated $T_{H} I$ cells produce proinflammatory cytokines such as IFN $\gamma$, forming a positive feedback loop for further $T_{H} I$ differentiation. ${ }^{15}$ This subset is important in eradicating infections mediated by intracellular pathogens. ${ }^{16}$ The cytokines produced by $T_{H}$ l-type cells are also important in enhancing macrophage antimicrobial activity to kill intracellular pathogens. ${ }^{2} \mathrm{~T}_{\mathrm{H}} \mathrm{I}$ subsets have also been identified as key role players in autoimmune diseases, particularly MS. ${ }^{17}$

$\mathrm{T}_{\mathrm{H}} 2$ type cells are the key subset in eliminating extracellular parasite infections through promoting eosinophil, basophil and mast cell function in addition to inducing B-cell class switching for lgE production. ${ }^{18} \mathrm{IL}-4$ determines $\mathrm{T}_{\mathrm{H}} 2$-type differentiation, signalling through the STAT6 and the transcription factor GATA-3.12 The major cytokine responses associated with $T_{H} 2$ immunity are IL-4, IL-5 and IL- I3.16 As with $T_{H}$ l subset dysregulation, an inappropriate $T_{H} 2$ response associated with IgE production can lead to the development of allergy and asthma. ${ }^{19}$

$\mathrm{T}_{\mathrm{H}} \mathrm{I} 7$ cells are characterised by their production of IL- I 7 and IL-22, and are a more recent addition to the T helper cell subset. ${ }^{13}$ This population arises in response to TGF $\beta$, IL-6 and IL-23, which induce the transcription factor ROR $\gamma$ T. ${ }^{18} \mathrm{~T}_{H} 17$ responses coordinate neutrophil function in destroying extracellular bacteria and fungi. ${ }^{18}$ In conjunction with $T_{H} I$ cells, $T_{H} 17$ subsets have been implicated in the pathogenesis of MS. ${ }^{20}$

\section{I.3.3. Regulatory $\mathrm{T}$ cells}

In contrast to effector $\mathrm{T}$ cell subsets, $\mathrm{CD} 4^{+}$Treg cells regulate the immune reaction by preventing the development of autoreactive immune responses. ${ }^{2}$ Tregs constitutively express CD25, the receptor for IL2, and can be further identified by the transcription factor FoxP3. ${ }^{13}$ FoxP3 interferes with the NFAT:API transcriptional complex on the IL-2 gene promoter, preventing the transcription of IL-2.21,22 Tregs can be further divided by type: natural Tregs which arise in the thymus, and induced Tregs, which develop in the periphery. Regardless, both Treg subsets share many phenotypic and functional characteristics. ${ }^{13}$ 
Tregs suppress other $\mathrm{T}$ cell functions through a number of mechanisms, primarily by interfering with interactions between APC and T cells. Through the production of IL-10, Tregs can dampen proinflammatory cytokine release by APC and inhibit antigen presentation on MHC class II molecules, preventing $T$ cell differentiation and effector functions..$^{23}$ In addition Tregs have been shown to compete with naïve T cells, blocking CTLA-4:B7 receptor interactions which are required for co-stimulation and activation of $\mathrm{T}$ cell effector functions. ${ }^{22}$ Finally, Tregs have been shown to sequester IL-2, a cytokine responsible for $\mathrm{T}$ cell proliferation. ${ }^{23}$ Because of their essential function in regulating the immune response, dysfunction of Treg responses is heavily implicated in the development of autoimmunity. ${ }^{24}$ 


\section{I.4. Central Nervous System Immune Privilege}

The brain and spinal cord are vital organs, essential not only for survival, but proper functionality of the body's other organs, consciousness, higher thought, and our unique experience as humans. Although inflammation serves as an important component in protecting the body from pathogens and maintaining homeostasis, it acts as a double-edged sword within the central nervous system (CNS). The brain is a particularly sensitive organ with limited capacity to repair, as a large proportion of the its cells exist in a post-mitotic state. ${ }^{25}$ Blood-borne macrophages and microglia, in particular are the principal effector cells responding to CNS disruption. ${ }^{26}$ Depending on their activation status and the pathogenic insult, macrophages that have infiltrated the brain release an array of pro-inflammatory mediators; cytokines, glutamate, free radicals and matrix metalloproteinases (MMP's). ${ }^{27,28}$ These factors promote apoptosis in the surrounding tissue, some of which cannot be repaired, resulting in scar tissue formation. ${ }^{29}$ It is well understood that neuronal processing depends on a series of activity-dependent pathways through key neuron circuitry, and that damage to, or removal of neurons in the system may lead to cognitive deficits. ${ }^{30}$

For its immediate protection, the brain is also encased in a rigid skull, which cannot accommodate excess cellular infiltrates or swelling, both of which are hallmark features of the inflammatory response. ${ }^{2}$ Cerebral edema is generally associated with poor neurological function in the long-term and is a strong independent predictor of mortality. ${ }^{31}$ Hence, the incentive to minimise inflammation within the CNS is strong.

Historically, the concept of CNS immune privilege has been ill-defined. The term describes the experimentally defined phenomenon wherein tissue transplanted into the CNS does not elicit an immune response against the foreign antigens and subsequent rejection as it would when grafted into peripheral tissue such as the skin. ${ }^{32,33}$ The secure nature of the CNS from the peripheral immune system, due in part to the impermeability of the blood brain barrier (BBB), choroid plexus (ChP), absence of traditional lymphatics, and apparent quiescence of microglia, the brain's resident macrophage population led scientists to believe that the CNS was immunogically inert and distinct from the periphery. ${ }^{34,35}$

It was not until some 30 years ago that a dramatic re-evaluation of the cellular responses within and surrounding the CNS began. ${ }^{36}$ While we now know that the CNS is immune privileged in that immune responses in the CNS is restricted, continuous immune surveillance occurs to protect the brain and spinal cord from infection. ${ }^{34}$ The immune responses in the CNS to injury or infection generally adopt a proresolving and pro-wound healing response to minimise tissue damage. A neuroprotective immune response is acute and self-limiting and results in pathogen elimination, tissue repair and the restoration of 
homeostasis in the CNS. Chronic neuroinflammation in the CNS results from dysregulation of the immune response and is a prominent feature of neurodegenerative diseases such as MS. These responses are aggressive and pro-inflammatory, resulting in oxidative stress, neuronal death, tissue atrophy and cognitive decline. In healthy individuals, two unique structures, the (BBB) and the ChP maintain the immune privileged state of the CNS. The BBB is an endothelial barrier that encompasses the cerebral vasculature, and is considered an absolute barrier, in which leukocytes crossing this structure constitute a hazard to the neural parenchyma. ${ }^{37}$ In diseases such as MS, BBB tight barrier function becomes dysregulated, and autoreactive lymphocytes exploit this structure to enter the CNS. ${ }^{38}$ In contrast, the choroid plexus can be considered an 'epithelial gate' into the CNS, allowing selective immune cell entry into the CNS. ${ }^{37}$ In contrast to the inert barrier function of the $\mathrm{BBB}$, the $\mathrm{ChP}$ appears to actively participate in modulating the immune responses occurring in the CNS. ${ }^{39,40}$ The primary focus of this thesis is the choroid plexus, which will be expanded upon below. 


\section{I.5. Multiple Sclerosis}

Multiple sclerosis (MS) is the most common cause of non-traumatic neurological disability in young adults, affecting approximately 2.8 million individuals worldwide, with twice as many women diagnosed versus men. ${ }^{41,42} \mathrm{MS}$ is a chronic, T-cell mediated inflammatory disease of the CNS that presents as focal demyelination, neuronal loss, gliosis and an aberrant inflammatory response mounted against CNS antigens. ${ }^{2}$ The most common form of the disease known as relapsing remitting multiple sclerosis (RRMS), is characterised by active immune-mediated lesion formation in the brain, followed by periods of subclinical disease where recovery occurs to baseline health levels. ${ }^{43}$ Over time, most RRMS patients progress to a secondary progressive form of the disease (SPMS) in which remission does not occur. 43 More rarely, patients are immediately diagnosed with primary progressive MS (PPMS), in which isolated immune attacks do not occur but instead disease symptoms culminate and progress until eventual death of the patient. ${ }^{17}$

The current literature offers little to explain why a nonspecific inflammatory event leads cells to attack self-antigens in the CNS. In the majority of cases, MS is not an immediately life threatening condition, but continuous autoimmune assault towards the CNS can lead to substantial disability through numerous sensory, motor, autonomic and neurologic dysfunctions. ${ }^{44}$ Disease onset generally occurs in the late twenties or early thirties, a key time for individuals to be establishing their independence, starting a family, becoming homeowners or excelling in their chosen career. MS robs these individuals of their quality of life, with up to $50 \%$ of patients requiring permanent mobility assistance just two decades after their diagnosis. ${ }^{17}$

\section{I.5.I. Risk factors}

Although the aetiology of MS is unknown, it is postulated that complex gene-environment interactions leads to the onset of disease pathology. The genetic components are likely responsible for the direct triggering of an autoimmune response, whereas environmental factors modulate activation thresholds and the overall inflammatory milieu. ${ }^{77}$ Genetic variation accounts for less than $30 \%$ of the overall risk factor with GWAS studies identifying little over 200 genes identified as 'risk regions', in which polymorphisms of these genes increases genetic predisposition in patients. ${ }^{17,45,46}$ Most of these genes code for molecules expressed on the immune system, with the most prominent gene identified as the human leukocyte antigen (HLA) on chromosome 6. The DRBI*I50I HLA class II allele variant has been consistently associated with increased susceptibility to MS in European/Pakeha, Asian and African American populations. ${ }^{47}$ The class I allele, $A * 02: 0$ I has also been shown to affect risk. It is also understood that polymorphisms in genes 
that code for T-cell activation and proliferation, namely IL-2 and IL7R, alongside other co-stimulatory factors, such as TNF $\alpha$ are key components in determining overall disease onset in MS patients, and even other immune disorders. ${ }^{48-50}$

Non-genetic factors have a larger contribution to overall MS incidence than genetic factors themselves, making up almost two-thirds of the risk. ${ }^{51}$ Without a common exogenous risk factor that links all MS patients, it is difficult to suggest whether MS is a disease that begins in the CNS or is a response triggered in the periphery that infiltrates the CNS. However there are numerous common risk factors that have repeatedly been shown to predispose individuals to MS; viral infection, vitamin D levels, smoking or obesity. ${ }^{17,46,51}$ Because there is no singular set of risk factors, there is no real way to prevent or lessen its socioeconomic impacts, making it a particularly difficult disease to manage.

\section{I.5.2. Immunopathology}

MS is widely believed be a disease of autoimmune origin, evidenced by the main target of pathology; myelin-protein derived antigens in the brain and spinal cord. The presence of autoreactive CD4+ $\mathrm{T}$ cells in the CNS is detectable from the earliest stages of MS. These T-cells recognise and orchestrate an immune attack towards several candidate autoantigens; myelin basic protein (MBP), myelin oligodendrocyte glycoprotein (MOG) and myelin associated glycoprotein (MAG) to cause neuronal damage in concentrated regions of the brain termed 'lesions' and the emergence of symptoms. ${ }^{43,52}$ Interestingly, CD4+ $\mathrm{T}$ cells harbouring TCR against these antigens have been found circulating in healthy individuals, however at a lower frequency than MS patients.53,54 In addition, these cells show a much higher avidity and proliferative response to myelin antigens in MS patients versus healthy, suggesting disease onset is associated with dysregulation of regulatory function in peripheral tolerance mechanisms. ${ }^{53,54}$ Although MS is considered to be a primarily T-cell-mediated disease, defective B cells, regulatory cells and macrophage/microglia have all been implicated at different time-points in disease pathology. ${ }^{17}$

The initial event that triggers the breakdown of tolerance and activation of autoreactive lymphocytes into aggressive effector subsets is unknown. The literature suggests that molecular mimicry, bystander activation, novel autoantigen presentation or the release of previously sequestered CNS antigen into the peripheral circulation may drive activation of these effector cells. ${ }^{17}$ For the activated immune cells to elicit damage in the CNS, they must migrate into the CNS through two unique tissues, the BBB and ChP. Proinflammatory factors relating to metabolic stress or infection may upregulate the expression of endothelial adhesion molecules in these tissues, facilitating the extravasation of lymphocytes from the 
systemic circulation into the CNS. ${ }^{52}$ In addition to $\mathrm{CD} 4^{+} \mathrm{T}$ cells, macrophages, and $\mathrm{CD} 8^{+} \mathrm{T}$ cells and dominate the early infiltrate. ${ }^{55}$ These cells direct damage towards myelin sheathes, the insulating layer that surrounds neurons. These lesions are generally self-limiting and there is little damage to the surrounding white or grey matter. ${ }^{17}$

As the disease progresses, demyelination becomes more diffuse throughout the brain, with notable ventricular atrophy and the formation of tertiary lymphoid structures in the meninges. ${ }^{17}$ In these latter stages, infiltration into the CNS reduces as chronic inflammation is well established. B cells make up a greater proportion of the pathogenic population, with microglia and macrophages in a state of chronic inflammation.

\section{I.5.3. Treatments}

Because of the autoimmune and degenerative nature of MS, there is no cure. For the majority of patients, who are afflicted with RRMS, there a broad range of disease modifying treatments (DMT's) available. These drugs are somewhat effective in managing relapses but will not prevent a transition to SPMS. ${ }^{56}$ These drugs are difficult to design because they must be effective against MS pathology but minimal impact on the normal protective function of the immune system. In NZ, both progressive forms of the disease have no existing treatments, options for these patients focus on the managing individual symptoms. ${ }^{57}$ Frontline treatments for RRMS in New Zealand include ocrelizumab, fingolimod, teriflunomide, dimethyl fumarate and natalizumab. ${ }^{57}$

\section{I.5.3.I. Natalizumab}

Natalizumab, sold under the brand name Tysabri ${ }^{\circledR}$ is a monoclonal antibody and one of the most successful treatments for MS. Natalizumab targets the migration of self-reactive lymphocytes into the CNS in the initial stages of disease. Effector lymphocytes extravasating into the CNS express integrin $\alpha_{4}: \beta_{1},(V L A-4)$. VLA-4 is the cognate ligand for vascular cell adhesion protein I (V-CAMI), upregulated on the inflamed luminal surface of endothelial cells of the blood-brain barrier.2,58,59 This interaction is required for the firm adhesion, attachment and migration of leukocytes into white matter lesions. Natalizumab is a highly specific VLA-4 glycoprotein antagonist, preventing the lock and key ligand-receptor interaction from occurring, denying self-reactive lymphocytes entry to the CNS. It was approved for use in RRMS patients following a successful clinical trial in 2004,60 but was pulled from the market briefly when a larger cohort study revealed serious and potentially fatal complications in a small percentage of patients. ${ }^{61,62} 1.5$ to 2.8 in every 1000 patients treated with natalizumab develop progressive multifocal leukoencephalopathy (PML). ${ }^{63} \mathrm{PML}$ 
is a devastating demyelinating disorder caused by the opportunistic infection of the John-Cunningham virus $(\mathrm{JCV})$. An estimated 39 to 58 percent of the general population have antibodies against the JCV, indicating a present or historical infection. ${ }^{63}$ Usually controlled by the immune system, in patients that are immunosuppressed, the JCV can undergo active replication in glial cells of the CNS. PML presents as a demyelinating disorder similar to MS, causing lytic damage to the oligodendrocytes of the CNS. The disease course is rapid and destructive, and primarily leads to death or severe long-term disability.62,63 Key risk factors for patients considering natalizumab are a seropositive result for JCV antibodies, previous use of immunosuppressants and the length of natalizumab therapy. 60 Because natalizumab significantly reduces relapse rates, expanded disability status scale (EDSS) score and the number of gadolinium-enhancing lesions, ${ }^{63}$ these key risk factors are considered by neurologists before natalizumab treatment is initiated.

\subsubsection{Ocrelizumab}

Ocrelizumab, clinically known as $\mathrm{Ocrevus}^{\circledR}$ is a recombinant humanized anti-CD20 monoclonal antibody that is a promising new treatment option for patients with RRMS and progressive forms of the disease.64,65 The drug targets B cells, an underestimated but key player in the pathology of MS. B cells mediate the disease by producing autoantibodies, self-antigen presentation, pro-inflammatory cytokine production and the formation of ectopic lymphoid tissues in the meninges. ${ }^{64,66,67}$ When treated with ocrelizumab, CD20expressing B cells are depleted through a number of cell-death pathways, although the mechanism by which this occurs is not well understood. Because CD20 is expressed on circulating immature, mature and memory B lymphocytes but not plasma and stem cells, B cells are not permanently depleted and will begin to self-replenish should the patient stop drug infusions. ${ }^{65}$ Following the first and second infusions at day I and I5, the drug is administered intravenously by a clinician once every 24 weeks, negating the need for regular visits to a hospital. In two phase III clinical trials of ocrelizumab versus an older, gold standard treatment; interferon- $\beta$ Ia in patients with RRMS, researchers found that in every aspect of clinical disease assessment, ocrelizumab outperformed the older treatment. At 12, and 24-weeks post treatment, ocrelizumab reduced relapse rate and confirmed disability progression (CDP) by up to 40 percent. Ocrelizumab is also presented as an alternative for patients on Tysabri ${ }^{\circledR}$ who have JCV positive CSF results, as there are currently no reported cases of PML with Ocrevus ${ }^{\circledR}$ treatment.67

\subsubsection{Fingolimod}

Fingolimod (FTY720), sold under the brand name Gilenya ${ }^{\circledR}$ was the first approved oral-DMT for RRMS patients in 2010 and is still a first line treatment in NZ. The mechanism of action of fingolimod differs 
greatly from other MS treatments, it has dual action by inducing lymphopenia and neuroprotection. Fingolimod is a synthetic immunomodulator, designed after the fungal metabolite, myriocin. ${ }^{68}$ It is a structural analogue to lipid sphingosine, the native sphingosine-I-phosphate receptor $\left(\mathrm{SIP}_{1,3,4,5}\right.$-receptor) ligand. These receptors affect a number of differential signalling pathways associated with cell migration, chemotaxis, survival, proliferation and activation. ${ }^{69,70}$ In particular, the SIPI-receptor is a G-protein coupled receptor expressed on CCR7+ immune cells, another key receptor involved in lymphocyte homing.71 A proposed model of lymphocyte circulation suggests that SIPI-receptors are required at exit sites in lymph nodes. ${ }^{72}$ SIPI-receptors allow lymphocytes to cycle between the blood and lymphoid organs, surveying organs for antigen before returning to the lymphatics and beginning again. SIP is found in the blood and lymph in higher concentrations than the lymph nodes, facilitating the egress of lymphocytes in response to a chemotactic gradient. 68,72

Fingolimod acts as a powerful functional antagonist for the SIPI receptor. ${ }^{73}$ SIPI-receptor lacking lymphocytes cannot respond to endogenous sphingosine-I-phosphate gradients and their egress from secondary lymphoid organs is prohibited. 2,73,74 Pathogenic cells that would otherwise extravasate into the CNS can no longer drain into the efferent lymphatics and exert their effects. The most prominent populations that Fingolimod sequesters in the lymph nodes are naive and central memory $\mathrm{T}$ cells, but the drug also exerts modest effects on B cells and NK cells. ${ }^{69}$ Both CCR7 negative effector T cells and effector memory $\mathrm{T}$ cells are not affected by the drug and may continue to circulate in the blood. ${ }^{69,75} \mathrm{~A}$ major caveat of fingolimod treatment is the broad expression of SIP receptors, leading to a number of (usually dosedependent) side effects which can be detrimental enough for patients to stop taking the therapy. ${ }^{73}$ In addition, concerns over PML in patients treated with fingolimod were confirmed, although at a lower frequency than natalizumab, at $<0.1$ per 1,000 patients treated.

\section{I.5.3.4. Teriflunomide}

Teriflunomide, known clinically as Aubagio ${ }^{\circledR}$ is a once-daily oral disease modifying therapy approved for the treatment of RRMS. Teriflunomide is a repurposed drug and the active metabolite of leflunomide; an immunomodulator on the market for rheumatoid arthritis. ${ }^{76}$ Teriflunomide targets proliferating lymphocytes by inhibiting pyrimidine synthesis. By specific, non-competitive and reversible inhibition of dyhydro-orotate dehydrogenase (DHODH), a mitochondrial enzyme, teriflunomide prevents proliferation of activated lymphocytes which requires de novo pyrimidine synthesis. ${ }^{77}$ Resting lymphocytes can still selfrenew using the pyrimidine salvage pathway but cannot undergo proliferation in response to activation 
signals. ${ }^{77}$ This ensures that normal homeostatic proliferation and immune surveillance can continue uninterrupted. ${ }^{78}$ Several other studies have described how teriflunomide acts through impairing the formation of immunological synapses, and decreases the release of IL-6, IL-8 and CCL2 from monocytes. ${ }^{79,80}$ In an open-label Phase III trial of teriflunomide in RRMS patients, researchers discovered that the drug reduced overall lymphocyte numbers, increased the ratio of $\mathrm{CD} 4^{+} / \mathrm{CD} 8^{+} \mathrm{T}$ cells and skewed $\mathrm{CD}^{+} \mathrm{T}$ helper differentiation in favour of Tregs, with a significant reduction in clonal diversity. ${ }^{81,82}$ Overall, this suggested a shift from pro-inflammatory responses to regulatory. In addition, patients could still mount an appropriate immune response, indicative that this drug is immunomodulatory not suppressive. ${ }^{81}$ Two phase III clinical trials (TEMSO and TOWER) have demonstrated that teriflunomide has positive effects on relapse rate, disability progression and MRI-determined outcomes.83,84 Overall, teriflunomide is well tolerated by patients, however was found not to be appropriate in patients with hepatic dysfunction, or in patients that were switching from other MS drugs; who had potential for rapid disease worsening. ${ }^{85}$

\section{I.5.3.5. Dimethyl Fumarate}

Tecfidera ${ }^{\circ}$, the MS drug with dimethyl fumarate (DMF) as an active ingredient is a twice-daily oral DMT approved for RRMS. DMF is a simple compound developed from fumaric acid. ${ }^{86}$ It has been used to treat psoriasis since the early 1990's, which shares an immune-mediated basis with MS. ${ }^{86}$ When taken orally, DMF is converted by gastrointestinal enzymes into its primary active principle, monomethyl fumarate where it exerts therapeutic effects. ${ }^{87}$ Although its' mechanism of action is widely unknown, DMF has been shown to have a multitude of biological targets. One of the major effects, shown both in vitro and in vivo is the capacity to shift the total immune response to an anti-inflammatory, $\mathrm{T}_{\mathrm{H}} 2$ response. ${ }^{88}$ Although $\mathrm{T}$ cells are the major population targeted, modulation of many other cell types including dendritic cells, macrophages, microglia, neurons and astrocytes have shown phenotypic changes related to anti-oxidative responses. ${ }^{89-91}$ Shifting the state of the immune system to an anti-inflammatory state has clear benefits in MS treatment, as evidenced by the two phase III clinical trials, DEFINE and CONFIRM. Both studies showed that annualized relapse was significantly reduced in patients taking DMF versus placebo, with positive effects on secondary endpoints including reductions in the number lesions, new or enlarging on T2-weighted MRI images.92,93 In October 2014, one case of fatal PML was reported due to long-term lymphopenia on DMF treatment, which must be taken into consideration for clinicians prescribing DMF.92,93 There is also limited data to suggest that DMF may show some teratogenicity in humans, due to its ability to cross the placenta, so is not appropriate for pregnant women. ${ }^{86}$ 
As previously described, disease modifying therapies for patients with RRMS are available, however their use may be limited by severe side effect profiles, namely PML associated with long term lymphopenia. No currently available treatments can cure the disease or consistently prevent the progression to secondary progressive MS.56 Given that most MS patients will rely on DMT's for the remainder of their lives, the cost to efficacy ratio is low. ${ }^{94}$ The market also lacks treatment options for patients with both progressive forms of MS, whose treatment focuses solely on managing symptoms. Thus, there is a clear need for novel, targeted therapies to help all of these patients.

\section{I.6. EAE as a model of MS}

Experimental autoimmune encephalomyelitis (EAE) has been a widely used experimental model for MS for decades. Indeed, several of the DMT's currently used for MS were developed in the EAE model first. ${ }^{95}$ Although this thesis does not involve any in vivo animal studies using EAE mice, future chapters explore the use of leukocytes derived from mice with active EAE disease. EAE is induced in mice by priming the peripheral immune system with myelin-specific antigens in an emulsion with complete Freund's adjuvant, which exacerbates the immune response to the myelin peptides.95,96 Similar to MS, the disease is primarily mediated by $\mathrm{T}$ cells, orchestrating an immune response against myelin that results in focal areas of demyelination in the central nervous system. 97 Other immune components including B cells, macrophages, microglia and DC are also implicated in disease progression. ${ }^{17}$ EAE manifests as paralysis that begins in the tail, moving upward to encompass the hind limbs, which can quantified on a disability scale from 0-5, (described in 2.2.3).98 


\section{I.7. The Choroid Plexus}

The $\mathrm{ChP}$ is a unique immunological interface that lies at the border between the blood and cerebral-spinal fluid (CSF). Nestled between the ventricles and folds of pia mater, the ChP is a convoluted structure comprised of specialised epithelial cells, called ependymal cells, and a stromal matrix underlying a dense network of fenestrated capillaries. ${ }^{37,99}$ The $\mathrm{ChP}$ is found in the lateral, third and fourth ventricles of both human and mice brains, following the corresponding choroidal fissure of its' respective ventricle. ${ }^{100}$

The fenestrated capillaries of the ChP provide little to no barrier function, indeed they act like the proximal tubules of the kidney, allowing free passage to large solutes and metabolites into the stromal space. 101 It is the tight junction molecules; occludins, claudins and cadherins at the apical surface of the ependymal cells that are tasked with forming the blood-CSF barrier (BCSFB). ${ }^{102}$

The ChP carries out a variety of functions; arguably the most important of which is the production and maintenance of the CSF. ${ }^{103}$ For many years, this was considered the primary and only function of the ChP. ${ }^{33}$ Recent studies have elucidated the key role the ChP plays as an immunological interface of the CNS, governing homeostatic trafficking into and out of the CNS.99 The stroma of the ChP houses a small population of tissue-resident APC's, poised for interaction with trafficking lymphocytes. . $^{9,104,105}$ Strominger et al. (2018), suggest that the ChP exists as an active immunomodulatory gate for bidirectional lymphocyte movement and as a niche for T cell stimulation, preconditioning the CNS for immune surveillance. ${ }^{39}$

Fenestrated capillaries in the $\mathrm{ChP}$ are easily traversed by leukocytes, which enter the $\mathrm{ChP}$ stroma. They rarely cross into the CSF due to the strong barrier properties of the ChP epithelia. ${ }^{39}$ Under normal conditions, the $\mathrm{ChP}$ epithelium produces an array of soluble mediators, that presumably govern which cells can translocate into the CSF. Like the CSF, the ChP adopts an immunosuppressive state under homeostatic conditions. ${ }^{37}$ At rest, the ependymal cells of the ChP constitutively express various chemokines; CXCLI0 and CCL20 in particular, are key mediators of chemoattraction and migration for cell-mediated immunity. ${ }^{106}$ At rest, these cells also release a slew of $\mathrm{T}_{\mathrm{H}} 2$ and $\mathrm{M} 2$-type cytokines, skewing any entering cells towards a pro-resolving milieu. ${ }^{107}$ 


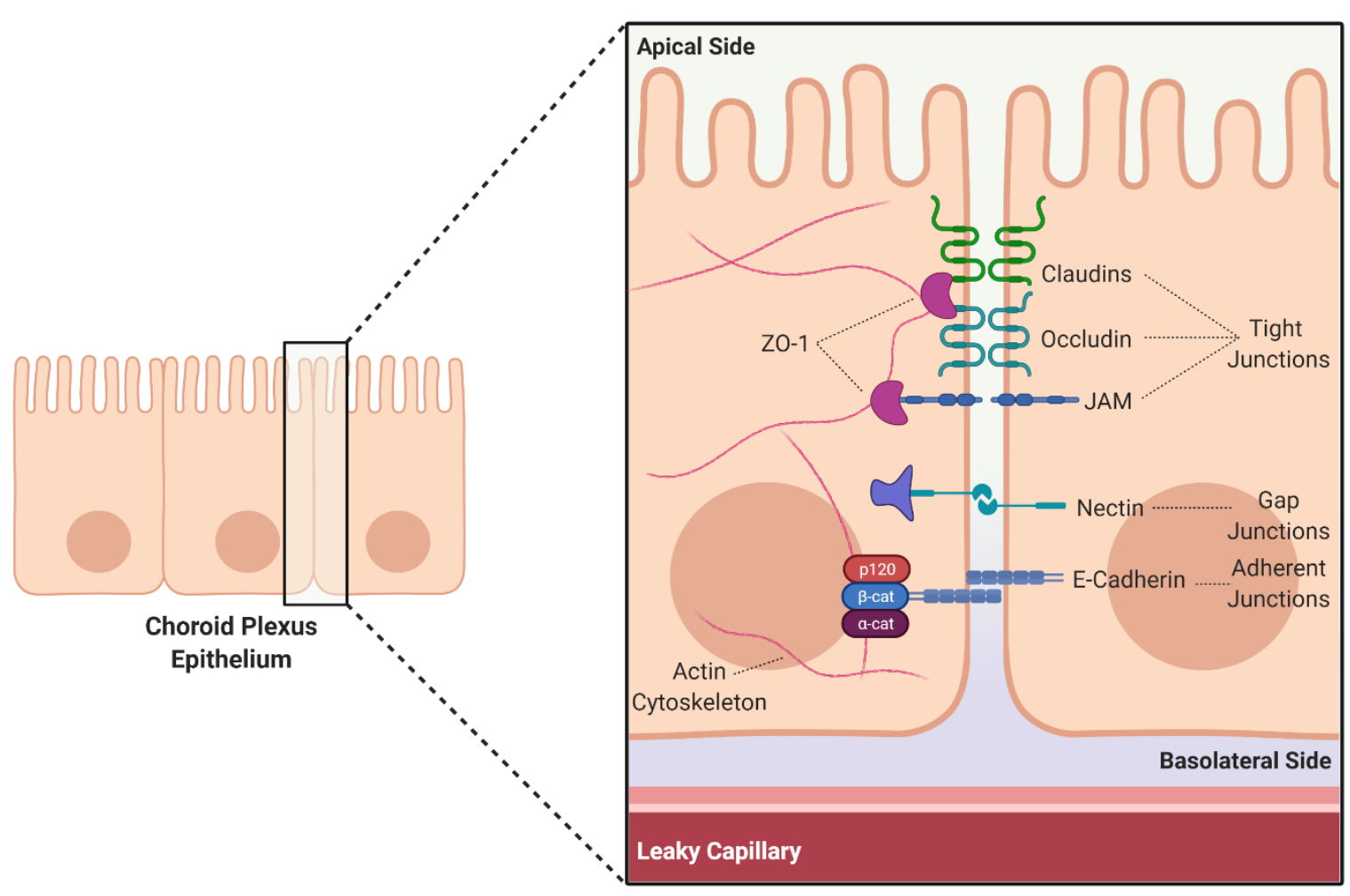

Figure I.I: The composition of intercellular molecules that maintain the blood-CSF barrier between polarized epithelial cells. Tight junction molecules: claudins, occludins and junctional adhesion molecules interact with zona occludins-I to form the apical-side tight barrier function. Gap junctions are comprised of nectin, which localises with E-cadherin on the basolateral side. E-cadherin forms adherens junctions through interactions with the intracellular armadillo family proteins; $\beta$-catenin and $p / 20$, which bind to $\alpha$-catenin, linking to the actin cytoskeleton. Image adapted from Tietz and Engelhardt., (2015). ${ }^{108}$

During inflammation, resident dendritic cells in the ChP stroma will interact with the pooled lymphocytes, presenting CNS antigens from CSF sampling. ${ }^{109}$ Indeed, one study showed that the majority of CD4+ T cells in the ChP stroma express TCR against neuroantigens in healthy individuals. ${ }^{110} \mathrm{~A}$ small population of resident $\mathrm{CD}^{+} \mathrm{T}$ cells live in the $\mathrm{CP}$ stroma, which primarily exhibit effector memory phenotypes and express $T_{H}$ I-type chemokine receptors, with high expression of IL-4, IFNY and FoxP3 versus those in circulation. ${ }^{110}$ These cells are thought to conduct regular neurosurveillance, offering a protective, regulatory role with immediate effector functions. Other $\mathrm{CD}^{+} \mathrm{T}$ cells transiently enter the ChP to pass through into the CSF, which adopt a central memory phenotype." " However, stromal dendritic cells have the capacity to skew the response of these $\mathrm{CD}^{+} \mathrm{T}$ cells, based on the inflammatory of tolerogenic phenotype they adopt. ${ }^{39}$ 
Interestingly, the $\mathrm{ChP}$ is newly implicated as a direct immunomodulatory gate in the pathogenesis of MS. $112-$ $115 \mathrm{It}$ is well understood that a breakdown of the BBB leads to the infiltration of autoreactive lymphocytes into the CNS, however the ChP is now suggested as the initial site of lymphocyte entry and priming of the inflammatory response. 116

In a two-way communication model, the ChP is also continuously exposed to T-cell-derived chemokines and cytokines. ${ }^{39}$ Such crosstalk between the $\mathrm{ChP}$ and its resident T-cell population may have the potential to alter the chemokine and adhesion molecule profile of the ChP epithelium during inflammation. ${ }^{109,117,118}$ Kunis et al., (2013) demonstrate that primary $\mathrm{ChP}$ cells respond to proinflammatory $T_{H} I$ type cytokines IFNY and TNFa by upregulating genes for chemokines associated with T-cell activation and trafficking, monocyte trafficking and increased expression of adhesion molecules. 119 Following the immune activation and homing of $T_{H} I$ and $T_{H} I 7$ cells into the CNS through the ChP, CCL5 and CXCL9-CXCLII are released by the epithelium and further propagate the lymphocyte recruitment process to this structure. ${ }^{39}$ High levels of CD68+ staining in the ChP stroma suggest the presence of activated macrophages, which are not typically found in healthy tissue. ${ }^{113,120} \mathrm{~T}$ cells in the ChP interact with resident monocyte and dendritic cell subsets, undergoing proliferation and activation given that their cognate peptide is presented. ${ }^{39} \mathrm{~T}_{H} \mathrm{I}$ activation in the ChP likely shifts the immune milieu, facilitating further invasion through this structure, and shifting the makeup of cell-mediated immunity in the CSF. Interestingly, adhesion molecules such as ICAM-I, VCAM-I, P-selectin and E-selectin are expressed apically on the ChP epithelium, rather than vasculature. $114,120-122$ These findings support the notion of bidirectional lymphocyte trafficking through this structure, and interactions between leukocytes homing to the $\mathrm{ChP}$ and resident immune cells that propagate peripheral inflammation throughout the CNS.39,119

The ChP constitutively produces CCL20, a CCR6 ligand, and a strong chemoattractant for $\mathrm{T}_{\mathrm{H}} \mathrm{I7} \mathrm{CD} 4^{+} \mathrm{T}$ cells. ${ }^{123}$ It is reported that CCL20 expression in the ChP mediates the initial step of CNS infiltration by autoreactive $\mathrm{T}_{H} 17$ lymphocytes in EAE. ${ }^{123}$ In a two-step model, Reboldi et al., (2009) describe how this initial infiltration triggers a CCL6-independent wave of parenchymal invasion by other immune cells. 37,123 Although, notably this tissue produces $C C L 20$, even in the absence of $T_{H} I 7$ cells in healthy individuals which acts a Treg chemoattractant during homeostasis, suggesting a role in neurosurveillance that becomes dysregulated in disease. ${ }^{37}$ In addition, macrophages entering the CNS in response to spinal cord injury through the ChP adopt a functionally distinct, regulatory M2 phenotype versus those that enter through the leptomeninges closer to the site of injury which adopt a classic, proinflammatory MI state. ${ }^{107}$ 
This suggests that entry through the $\mathrm{ChP}$ can affect the fate of leukocytes by skewing them towards a more regulatory phenotype. In healthy individuals, the $\mathrm{ChP}$ may indeed act as a regulatory structure to promote an active, CNS-protective response despite the state of the peripheral inflammatory response.

Although it is now clear that the ChP acts as a permissive gate for leukocyte trafficking into the CNS, the mechanisms by which it becomes dysregulated in diseases such as MS are unclear. Certainly, the ChP plays a role in MS pathogenesis, it is an inherently leakier tissue than the BBB so many autoreactive lymphocytes penetrate this structure and pool in the CSF. MRI of patients with MS and neuromyelitis optica spectrum disorder show significant enhancement in thickness of the ChP. ${ }^{124}$ Both pathogenic T and B cells have been shown to exploit the ChP to gain entry to the CNS during active MS. ${ }^{125,126}$ Because the ChP has not been a focal point of neuroimmunology, we fail to understand the mechanisms by which these cells gain entry to the CNS. Understanding of these mechanisms will provide opportunity to develop novel therapeutic interventions that target these processes, allowing us to control inflammatory responses in the CNS and promote repair.

\section{I.8. Heparanase/heparan sulfate}

Heparan sulfate proteoglycans (HSPG's) are the most abundant components of the extracellular matrix (ECM), playing a major role in maintaining tissue architecture and integrity. ${ }^{127}$ Heparan sulfates $(\mathrm{HS})$ are complex linear polysaccharide chains, bound heavily by sulfate. ${ }^{127}$ They exist either as free unconjugated chains, or covalently bound to protein cores in the extracellular matrix. HSPG's are involved in a number of complex biological pathways that propagate cell signalling, adhesion, migration and can act as a depot for growth factors. ${ }^{128}$

Mammalian heparanase is an endo- $\beta$-D-glucuronidase responsible for cleaving heparan sulfate chains. It is synthesized as a large latent precursor $(65 \mathrm{kDa})$ and undergoes proteolytic processing in the acidic lysosome environment, yielding $8 \mathrm{kDa}$ and $50 \mathrm{kDa}$ subunits that associate to form a heterodimer. ${ }^{129,130}$ Because the processed enzyme becomes highly active, it is under strict regulation. 128,131,132 Heparanase is constitutively inactive in most cell types, its expression is restricted to platelets, leukocytes and the placenta, with no expression in healthy connective tissues and epithelia. ${ }^{128,132}$ The heparanase-coding gene is under promoter methylation and the inhibitory activity of p53 in homeostatic conditions.

Heparanase has been implicated as a major player in the pathogenesis of cancer, regulating angiogenesis and metastasis. Cancers with elevated levels of heparanase expression are generally associated with an 
aggressive phenotype and poor post-operative survival rate. Thus, heparanase has gained prominence in cancer biology as a potential anti-neoplastic drug. Research in this area is still relatively new, with promising results in a number of drug candidates as an adjuvant to chemotherapy. Emerging evidence also suggests a role for heparanase in other chronic inflammatory conditions, such as MS. 133,134

During inflammation, upregulated heparanase activity cleaves native heparan sulfate molecules present in the ECM, which breaks down the matrix integrity and destabilises the underlying endothelial cell barrier. ${ }^{130}$ In cancer, this well-described role facilitates angiogenesis and metastasis. 130 In terms of cell signalling, this process modulates the interactions of chemokines, cytokines, and other signalling molecules with their receptors. Heparanase activity can release HS-sequestered proteins, leading to the formation of chemokine gradients at the BBB. ${ }^{135}$ This can facilitate trafficking of inflammatory leukocytes through the disrupted endothelial monolayer. ${ }^{135}$ In addition to changes in leukocyte trafficking into the CNS during inflammation, heparanase has also been shown to regulate the activation of microglia and astrocytes. ${ }^{133}$ Its involvement in similar CNS disorders suggests that heparanase may govern some of the early neuroinflammatory events that occur in MS, including the trafficking of autoreactive immune cells across CNS barriers.

Indeed, heparanase has been a target of MS research for some time now, there is significant literature to support the role that heparanase contributes to disease pathology in EAE and MS. Willenborg and Parish., (1988) showed that administration of heparin and fucoidan, two highly sulfated polysaccharides completely inhibited the onset of adoptive transfer EAE, and the total absence of lesions in the CNS. Parish suggested that heparin and other sulfated polysaccharides strengthened the ECM scaffolding by occupying and blocking the active site of heparanase, expressed by lymphocytes emigrating into the CNS.136,137 Subsequent studies confirmed this by showing that low-dose modified heparins led to complete inhibition of adoptively transferred EAE in Lewis rats. ${ }^{138}$ This was attributed to the specific inhibitory effect on Tcell heparanase expression in relation to cell trafficking, which provided rationale for its use as an immunomodulator. ${ }^{138}$ In 1990, this group also established concanavalin (ConA) stimulation in vitro induces T cell heparanase expression, which is associated with the ability of these cells to penetrate vascular endothelium into target tissues. ${ }^{139}$ This could be inhibited with low dose heparin at $0.1 \mu \mathrm{g} / \mathrm{mL}$ and in vivo, heparin completely inhibited the capacity of lymphocytes to migrate to the antigenic site to elicit an immune response. ${ }^{139}$ 
Because heparanase expression in T cells has been shown to be upregulated in response to in vitro MBP stimulation, it can be hypothesized that heparanase expression is upregulated in lymphocytes from MS patients, contributing to an overall increase in T-cell aggressive phenotype. ${ }^{136}$ de Mestre et al., (2007) confirm this using in vivo studies with a rat EAE model; heparanase mRNA expression was found to be induced in antigen-specific CD4+ T cells and it's expression directly correlated to the cells' encephalitogenicity. Interestingly, while heparanase was highly expressed in the CD4+ cells infiltrating the CNS of these rats, little to no expression was measured in $\mathrm{CD} 8^{+} \mathrm{T}$ cells, macrophages/microglia and astrocytes during active disease. 140 In addition, T-cell heparanase, even devoid of its enzymatic activity has been shown to have pro-adhesion properties, mediating rolling and arrest during sheer flow conditions. ${ }^{141}$ Together, this data suggests that heparanase plays a major role in establishing and maintaining disease progression in MS.

The current literature sets out a clear rationale for the role of heparanase in MS disease progression. In short, virtually all cerebral blood vessels express at least one class of HSPG's on their surface. ${ }^{142}$ Activated CNS-antigen specific CD4+ $\mathrm{T}$ cells and monocytes exploit the enzymatic and pro-adhesion properties of

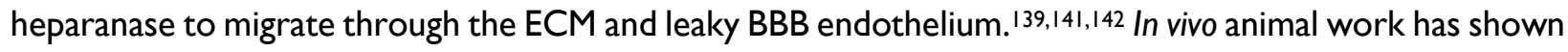
that blocking ECM degradation is protective in EAE, and overexpressing heparin prevents innate immune cell and T-cell infiltration into the CNS.137,142,143 In addition, heparan sulfate mimetics have been shown to be powerful inhibitors of $T_{H} I / T_{H} I 7$ type effector functions, skewing instead towards a FoxP3+ Treg response. ${ }^{144}$ Because of its well-described role, heparanase is an attractive target for drug development pathways 


\section{I.9. HSI6-35}

Because heparan and heparan sulfate mimetics have previously been shown to inhibit migration across the barrier structures into the CNS, we propose that HSI6-35 will inhibit migration across an experimental model of the ChP. 'HSI6-35' is a single entity HS-glycomimetic cluster, which is part of a larger, targeted library of HS-mimetics conjugated to simple sulfated saccharides developed by the Zubkova lab at the Ferrier Research Institute. Many labs across the world have developed HS mimetics, which interfere with heparanase activity, but their development is often hindered by the size and complexity of the molecules..$^{45}$ These glycomimetics are smaller in size and have a much shorter synthesis, making them accessible and commercially viable. $\mathrm{HSI}$-35 shows potent heparanase inhibition with low $\mathrm{IC}_{50}$ values, comparable to competitors in development. In early cancer research, these compounds display stunning anti-cancer properties, inhibiting growth and metastasis of human myeloma tumour cells and angiogenesis. 145 More interestingly, HSI6-35 reportedly lacks anticoagulant activities and cytotoxicity, a major issue of other HSmimetics in clinical development. ${ }^{146}$

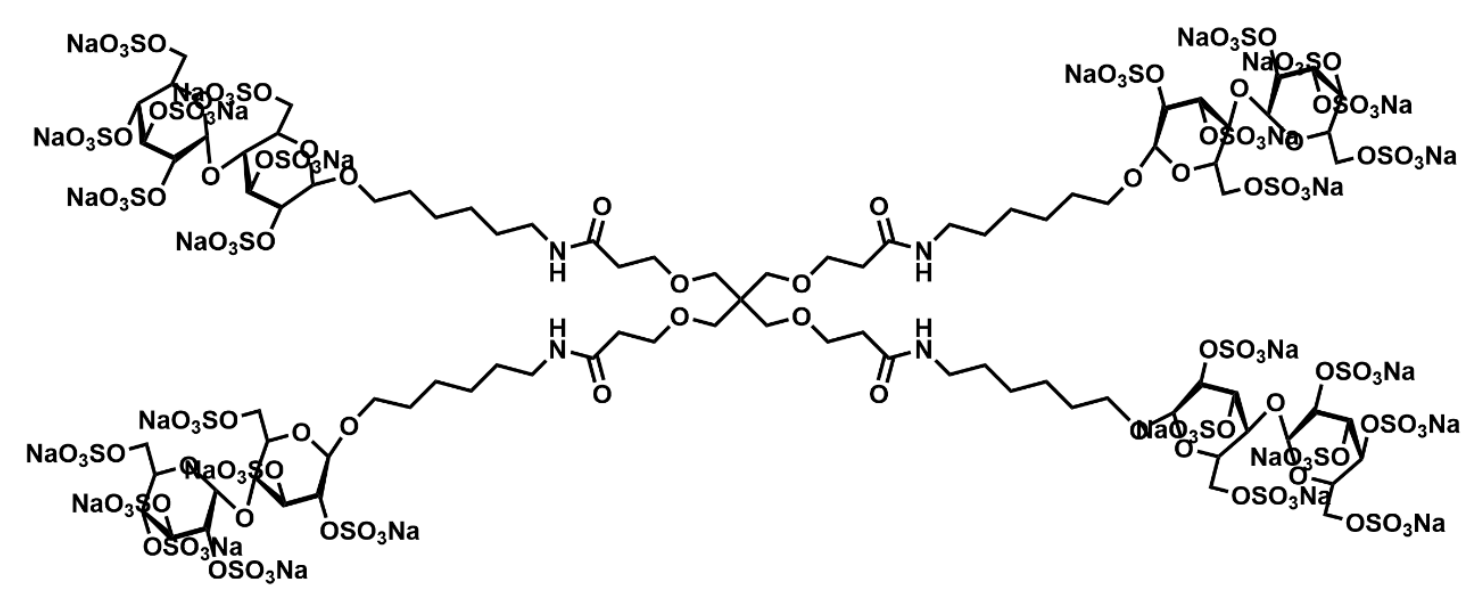

Figure 1.2: The chemical structure of HSI6-35. HSI6-35 is comprised of a dendritic core with four identical sulfated oligosaccharide arms. 


\section{I.I 0. Research Aims}

The aim of this research was to establish whether the novel HS-mimetic, HSI6-35 could inhibit the migration of immune cells across a model of the ChP. First, this project required the establishment of an experimental in vitro model of the ChP. This model was capable of mimicking both homeostatic and inflammatory trafficking into the CNS. Next, the cytotoxicity of HSI6-35 on murine splenocytes and ChP epithelium was assessed to determine the optimum concentration to treat cultures in vitro. Using this migration model, I assessed the efficacy of low-dose HSI6-35 to inhibit migration across the resting and inflamed ChP epithelium. Finally, to understand whether HSI6-35 modulates lymphocyte migration through other mechanisms, I investigated the cytokine profiles of murine splenocytes and ChP epithelium in response to HSI6-35 treatment under both homeostatic and inflammatory conditions. In summary, my specific research aims were:

I. To establish an experimental in vitro model of the ChP capable of mimicking both homeostatic and inflammatory trafficking.

2. To determine the optimum concentration of HSI6-35 for use in this model system.

3. To evaluate the ability of HSI6-35 to inhibit migration across resting and inflamed ChP epithelium.

4. To investigate the cytokine profiles of murine splenocytes and ChP epithelium in response to HSI6-35 treatment under both homeostatic and inflammatory conditions. 
Chapter I: General Introduction 

Chapter 2: Materials and Methods 


\section{I. Materials}

\section{I.I. Table of Materials}

\begin{tabular}{|c|c|c|}
\hline Item & $\begin{array}{l}\text { Catalog } \\
\text { Number }\end{array}$ & Company \\
\hline 2-Mercaptoethanol & $21985-023$ & $\begin{array}{c}\text { Life } \\
\text { Technologies }\end{array}$ \\
\hline $\begin{array}{l}6.5 \mathrm{~mm} \text { Transwell } 囚 \text { with } 8.0 \mu \mathrm{m} \text { Pore Polyester Membrane Insert, } \\
\text { Sterile }\end{array}$ & 3464 & Corning \\
\hline Collagen, Type I solution from Rat Tail & C3867 & Gibco \\
\hline $\begin{array}{l}\text { Concanavalin A from Canavalia ensiformis, Type IV-S, lyophilized } \\
\text { powder, }\end{array}$ & C5275 & Sigma Aldrich \\
\hline Corning® Matrigel $囚$ Basement Membrane Matrix, LDEV-free, & 354234 & Corning \\
\hline Dimethyl Sulfoxide (DMSO) Hybri-Max ${ }^{\mathrm{TM}}$, Sterile-filtered & D2650 & Sigma Aldrich \\
\hline DPBS (IOX), no calcium, no magnesium & $14200-075$ & Gibco \\
\hline Dulbecco's Modified Eagle Medium (DMEM), high glucose, pyruvate & $11995-073$ & ThermoFisher \\
\hline Evans Blue Dye & E2I29-I00G & Sigma Aldrich \\
\hline Fetal Bovine Serum, New Zealand Origin, Sterile Filtered & F8067, Lot I6A327 & Sigma Aldrich \\
\hline Freund's Adjuvant, Complete & F588I & Sigma Aldrich \\
\hline HEPES (IM) & $15630-080$ & ThermoFisher \\
\hline InVivoMAb anti-mouse IFN $\gamma$ & BE0055 & BioXCell \\
\hline L-Glutamine (200mM) & $25030-081$ & ThermoFisher \\
\hline MEM Non-Essential Amino Acids Solution (I00X) & $11140-050$ & ThermoFisher \\
\hline $\begin{array}{l}\text { Millex-GP Syringe Filter Unit, } 0.22 \mu \mathrm{m} \text {, polyethersulfone, } 33 \mathrm{~mm} \text {, } \\
\text { gamma sterilized }\end{array}$ & FPE-204-030 & Interlab \\
\hline MTT (3-(4,5-Dimethylthiazol-2-yl)-2,5-Diphenyltetrazolium Bromide) & M6494 & ThermoFisher \\
\hline Myelin Oligodendrocyte Glycoprotein Peptide (35-55), mouse, rat & RPI0245 & Genescript \\
\hline Penicillin-Streptomycin $(10,000 \mathrm{U} / \mathrm{mL})$ & $15140-122$ & ThermoFisher \\
\hline Pertussis toxin from $B$. pertussis & 181 & $\begin{array}{l}\text { List } \\
\text { Biochemicals }\end{array}$ \\
\hline Recombinant Mouse Rantes (CCL5) & $\mathrm{CHM} 342$ & Prospec \\
\hline Recombinant Murine IFN $\gamma$ & $315-05$ & PeproTech \\
\hline Red Blood Cell Lysing Buffer Hybri-Max ${ }^{\mathrm{TM}}$ & R7757 & Sigma Aldrich \\
\hline Trypan Blue Solution, $0.4 \%$ & $|5250-06|$ & Gibco \\
\hline TrypLE TM Express Enzyme (IX), no phenol red & $|2604-02|$ & Gibco \\
\hline
\end{tabular}




\subsection{Animals and Tissue Harvest}

\subsection{Animals}

8-week-old C57BL/6] mice were purchased from the Biomedical Research Unit (BRU) at the Malaghan Institute for Medical Research (Wellington, New Zealand). Animals were housed in a specific-pathogenfree facility on a I2-hour light/ 2-hour dark cycle, and ad libitum access to food and water for the duration of all experiments.

\subsubsection{Ethics declaration}

All experimental measures were approved by the Victoria University of Wellington Animal Ethics Committee under Ethics Application \#25295 (20-09-2017). Experiments were performed in accordance with all relevant guidelines and procedures at the School of Biological Sciences Animal Facility (Victoria University of Wellington).

\subsubsection{EAE induction in animals}

Myelin Oligodendrocyte Glycoprotein ( $\mathrm{MOG}_{35-55}$ peptide) (Genescript) emulsions were prepared at a concentration of $50 \mu \mathrm{g} / 200 \mu \mathrm{L}$ per mouse which included $500 \mu \mathrm{g}$ heat-inactivated Mycobacterium tuberculosis in complete Freund's adjuvant. Pertussis toxin (List Biochemicals) was administered at a concentration of $200 \mathrm{ng}$ per mouse, prepared in $200 \mu \mathrm{L}$ Pertussis toxin buffer (2.14.9). $100 \mu \mathrm{L}$ MOG emulsion was injected subcutaneously into each leg using a 23G needle, followed by $200 \mu \mathrm{L}$ pertussis toxin injected intraperitoneally. Mice were weighed and scored daily for motor deficits and ascending paralysis using a numerical scale as follows: 0- no disease, 0.5 - loss of tail tonicity, I.0- half-tail paralysis, I.5- threequarter tail paralysis, 2.0- full tail paralysis, 2.5- full tail paralysis and hind limb weakness, 3.0 one hind limb paralysed or severe weakness in both hind limbs, 3.5- on hind limb paralysed and severe weakness in the other hind limb, 4.0- full hind limb paralysis and front limb weakness, 5.0- moribund/death. Mice losing $>20 \%$ of their starting weight or surpassing a score of 4.5 with no active recovery were euthanised.

\subsubsection{Tissue Isolation}

Mice were euthanised by lethal carbon dioxide asphyxiation in a euthanasia chamber. Death was verified by establishing the loss of reflexes and the presence of fixed and dilated pupils. Following death, mice were thoroughly swabbed with $70 \%$ ethyl alcohol in $\mathrm{ddH} 2 \mathrm{O}$. Splenic tissue was harvested and placed in I $\mathrm{mL}$ Wash Buffer (DMEM supplemented with 3\% HEPES, I\% Penicillin-Streptomycin) and placed on ice until further use. 


\subsubsection{Splenocyte isolation}

Spleens were mechanically digested into a single cell suspension via forced passage through a $70 \mu \mathrm{m}$ cell strainer using Wash Buffer, and the thumb-end of a plunger fitting a I mL syringe (BD). The cell suspension was centrifuged at $700 \mathrm{xg}$ for three minutes. The cell pellet was re-suspended in $2 \mathrm{~mL}$ Red Blood Cell Lysis Buffer (Sigma Aldrich). Cells were incubated at room temperature for 2 minutes and lysis was terminated by the addition of $8 \mathrm{~mL}$ Wash Buffer, followed by centrifugation again at $700 \mathrm{xg}$ for three minutes. Isolated splenocytes were then re-suspended in $10 \mathrm{~mL}$ CTCM (DMEM supplemented with $10 \%$ Fetal Bovine Serum (FBS), I\% Penicillin-Streptomycin, I\% HEPES, I\% Non-essential amino acids, I\% L-glutamine and 0.0।\% Beta-mercaptoethanol).

\subsubsection{Splenocyte viability assessment}

To assess the percentage of live splenocytes, cells were resuspended in CTCM as described in 2.2.5. Splenocytes were diluted in a I:I0 ratio in trypan blue dye (Sigma). I0 $\mu \mathrm{L}$ of this cell-dye solution was added into a Neubauer haemocytometer with coverslip (Hawksley), and cells were counted using a compound microscope. Using this assessment, splenocytes were diluted in CTCM to a concentration of $1 \times 10^{7}$ cells $/ \mathrm{mL}$. 


\subsection{General Epithelial Cell Culture}

\subsection{ECPC-4 cell culture}

The transformed C57BL/6 Mus musculus Epithelial Choroid Plexus Cell (ECPC-4) cell line (RRID: CVCL_4836) was purchased through Riken Cell bank from Fukuoka University, Fukuoka, Japan. ECPC-4 cells are immortalized via transfection with $\operatorname{lgH}$ enhancer-SV40 $\mathrm{T}$ fusion gene. All cell culture was conducted in a sterile, class II laminar flow hood. Cells were cultured as recommended by the manufacturer, at $\mathrm{I} \times 10^{6}$ cells $/ \mathrm{mL}$ in T75 flasks (Corning) in $20 \mathrm{~mL}$ Complete Growth Media (DMEM supplemented with 10\% FBS and 1\% Penicillin/Streptomycin). Cultured cells were incubated at $37^{\circ} \mathrm{C}$, $5 \% \mathrm{CO}_{2} \mathrm{RH}$.

\subsubsection{Thawing Cells}

Vials were removed from liquid nitrogen storage and placed into a $37^{\circ} \mathrm{C}$ water bath to thaw. Under a sterile laminar flow hood, cells were transferred to a falcon tube containing $9 \mathrm{~mL}$ Complete Growth Media. The vial was washed thoroughly with another I $\mathrm{mL}$ growth medium to recover the maximum number of cells. To remove the freezing medium, the tube was centrifuged at $400 \mathrm{xg}$ for five minutes, and the pellet was re suspended in $10 \mathrm{~mL}$ growth media. The cells were then transferred to a T25 flask at a concentration of $2 \times 10^{6}$ cells/flask (in $10 \mathrm{~mL}$ complete growth media) and incubated for 24 hours at $37^{\circ} / 5 \% \mathrm{CO}_{2} \mathrm{RH}$.

\subsubsection{Passaging Cells}

At $80 \%$ confluency, as assessed visually, cells were subcultured with $0.25 \%$ Trypsin (Sigma) at a passage ratio of 1:4 into fresh T75 flasks. Cells were used up to and including Passage 20. Growth media was removed from the flask via vacuum aspiration, and cells were gently washed with $10 \mathrm{~mL} I X \mathrm{dPBS}$ (Gibco). The dPBS was aspirated, followed by the addition of $3 \mathrm{~mL}$ Tryple $®$ (ThermoFisher) to the flask. Cells were incubated at $37^{\circ} / 5 \% \mathrm{CO}_{2} \mathrm{RH}$ for I minute. Upon removal from the incubator, the flask was gently tapped to encourage cells to detach. $7 \mathrm{~mL}$ growth medium was then added to the flask using a serological pipette with a blasting technique to detach any remaining cells from the surface of the plastic. Cells were then centrifuged at $400 \times g$ for 3 minutes and re-suspended in $10 \mathrm{~mL}$ growth media. Trypan-blue exclusion dye in a 1:10 ratio was used to assess viability and cell concentration. 


\subsubsection{Freezing Cells}

Cells were centrifuged at $400 \mathrm{xg}$ for 3 minutes, re-suspended in $10 \mathrm{~mL}$ freezing medium (10\% DMSO in $90 \%$ FBS) and adjusted to a concentration of $1 \times 10^{6}$ cells $/ \mathrm{mL}$. The cell solution was transferred to cryovials in $2 \mathrm{~mL}$ aliquots. These vials were placed into an IPA-lagged freezing container and stored at $-80^{\circ} \mathrm{C}$ for 24 hours. The vials were then transferred to liquid nitrogen for long term storage. 


\subsection{Establishing a Transwell Monolayer Culture}

These methods discuss the optimised conditions that the Transwell experiment was run under. A major part of this thesis research was establishing the model, and in doing so, finding the best conditions to mimic in vivo migration. An appendix methods section can be found after this chapter, which includes methods used in setting up this experiment, but were not successful and/or not used in the final model. This will provide a concise methods section here, and extra information to support the results and discussion chapters.

\subsection{Preparation of Transwells for Cell Culture}

\subsection{I.I. Collagen coating}

Inverted Transwells were incubated with $75 \mu \mathrm{L}$ I mg/mL Rat Tail Collage Type I (Sigma Aldrich) under a tissue culture hood for 2 hours. Excess collagen was gently pipetted from surface, the Transwells were rinsed twice with IX dPBS (Thermo Fisher) and left to dry under the hood. To avoid contamination, Transwells were sterilised under UV light for 30 minutes prior to seeding.

\subsubsection{Seeding ECPC-4 Cells onto Transwells}

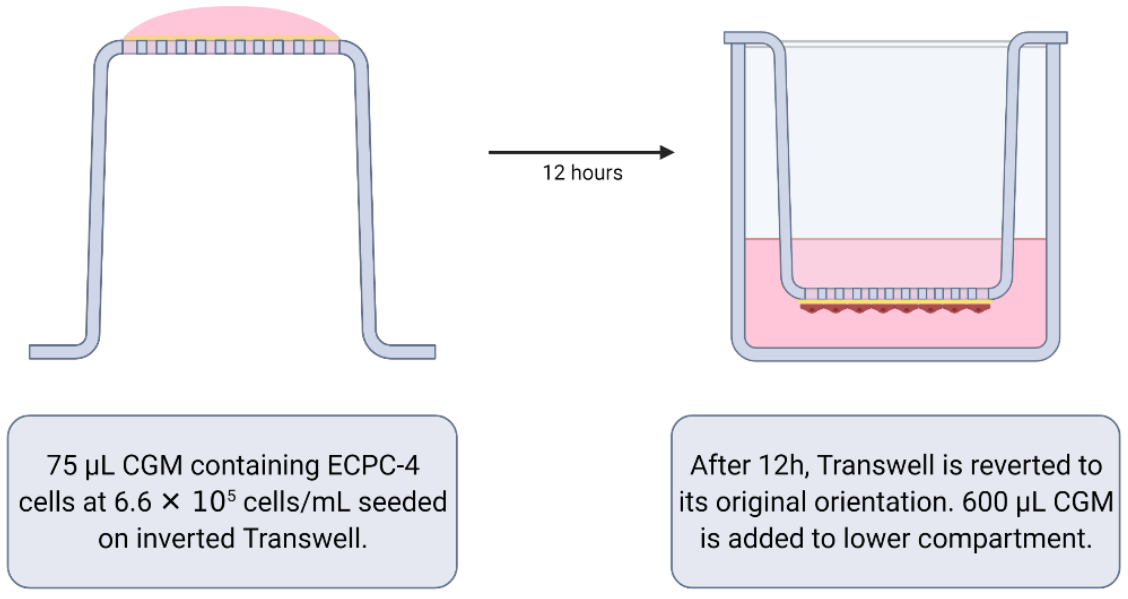

Figure 2.I: Simplified schematic showing how we seed ECPC-3 cells onto a Transwell. ECPC-4 cells were passaged and re-suspended in a $50 \mathrm{~mL}$ conical centrifuge tube (Falcon) as described in

2.3. The concentration of cells was adjusted to $6.6 \times 10^{5}$ cells $/ \mathrm{mL}$ in warm CTCM. Cells were seeded at a volume of $75 \mu \mathrm{L}$ to the inverted mesh of the Transwell (absolute cell number $=5 \times 104$ ). To promote adherence, cells were incubated in this inverted orientation for $12 \mathrm{~h}$. At $12 \mathrm{~h}$, the Transwells were gently oriented back into an upright position using sterilized tweezers. To the lower compartment of the 
Transwell culture, $600 \mu \mathrm{L}$ complete growth media was added; enough to generously submerge the ECPC4 monolayer.

\subsubsection{Splenocyte Stimulation}

\subsubsection{Concanavalin (ConA)}

Splenocytes were seeded at I $\times 10^{7}$ cells $/ \mathrm{mL}$ in $100 \mu \mathrm{L}$ aliquots into a 96 well plate. For stimulation with ConA, cells were treated at I $\mu \mathrm{g} / \mathrm{mL}$ in a total of $200 \mu \mathrm{L}$, containing CTCM, splenocytes and ConA. For Transwell experiments, splenocytes were stimulated for a total of $24 \mathrm{~h}$ at $37^{\circ} \mathrm{C} / 5 \% \mathrm{CO}_{2} \mathrm{RH}$. Media containing ConA was removed following centrifugation of the plate at $400 \mathrm{xg}$ for 4 minutes. Splenocytes were re-suspended in $200 \mu \mathrm{L}$ fresh CTCM without ConA for $24 \mathrm{~h}$ before addition to the Transwell culture.

\subsubsection{Interferon- $\gamma($ IFN $\gamma)$}

Splenocytes were seeded at I $\times 10^{7}$ cells $/ \mathrm{mL}$ in $100 \mu \mathrm{L}$ aliquots into a 96 well plate. For stimulation with IFN $\gamma$, cells were treated at $20 \mathrm{U} / \mathrm{mL}$ in a total of $200 \mu \mathrm{L}$, containing CTCM, splenocytes and IFN $\gamma$. Cells were stimulated with IFN $\gamma$ for a total of $48 \mathrm{~h}$ at $37^{\circ} \mathrm{C} / 5 \% \mathrm{CO}_{2} \mathrm{RH}$. Supernatant containing IFN $\gamma$ was not aspirated before adding splenocytes into the Transwells.

\subsubsection{Anti-interferon- $\gamma(\alpha I F N \gamma)$}

Anti-IFN $Y$ was added to splenocyte cultures following the removal of ConA, as described in 2.4.2.I. For designated wells, anti-IFN $\gamma$ antibody was added at a concentration of $10 \mu \mathrm{g} / \mathrm{mL}$ in a total volume of $200 \mu \mathrm{L}$, containing CTCM, splenocytes and anti-IFNy. Cells were then directly transferred into the Transwell culture. In the Transwells, splenocytes were treated with anti-IFN $\gamma$ for a total of $24 \mathrm{~h}$.

\subsubsection{Treatment of the Transwell Culture with HSI5-35}

\subsubsection{Splenocyte Treatment}

Splenocytes were seeded at $\mathrm{I} \times 10^{7}$ cells $/ \mathrm{mL}$ in $100 \mu \mathrm{L}$ aliquots into a 96 well plate. Designated wells for HSI6-35 (Ferrier Research Institute, NZ) may also include ConA, IFNY and/or anti-IFNY. HSI6-35 was incubated in the splenocyte culture at $60 \mu \mathrm{g} / \mathrm{mL}$ in a volume of $200 \mu \mathrm{L}$ for $48 \mathrm{~h}$ prior to addition to the Transwell. Media containing HSI6-35 was aspirated following centrifugation of the plate at $400 \mathrm{xg}$ for 4 minutes. In wells pre-treated with ConA, splenocytes were re-suspended in fresh CTCM + HSI6-35, omitting ConA before addition to the Transwell culture. Wells treated with HSI6-35 minus ConA did 
not receive replacement media. In total, all splenocyte wells were cultured with HSI6-35 for a total of $72 \mathrm{~h}$.

\subsubsection{Epithelial Monolayer Treatment}

ECPC-4 cells that had successfully formed a monolayer culture on the Transwells (occurs approximately $\sim 24 \mathrm{~h}$ after cells seeded) were treated with $\mathrm{HSI} 6-35$ at $60 \mu \mathrm{g} / \mathrm{mL}$ for $24 \mathrm{~h}$ prior to the addition of splenocytes to the upper well. Upon starting the Transwell culture, and the addition of the CCL5 cytokine, media containing fresh HSI6-35 was added. In total, the epithelial cells were treated with HSI6-35 for $48 \mathrm{~h}$. 


\subsection{Transwell Migration Assay}

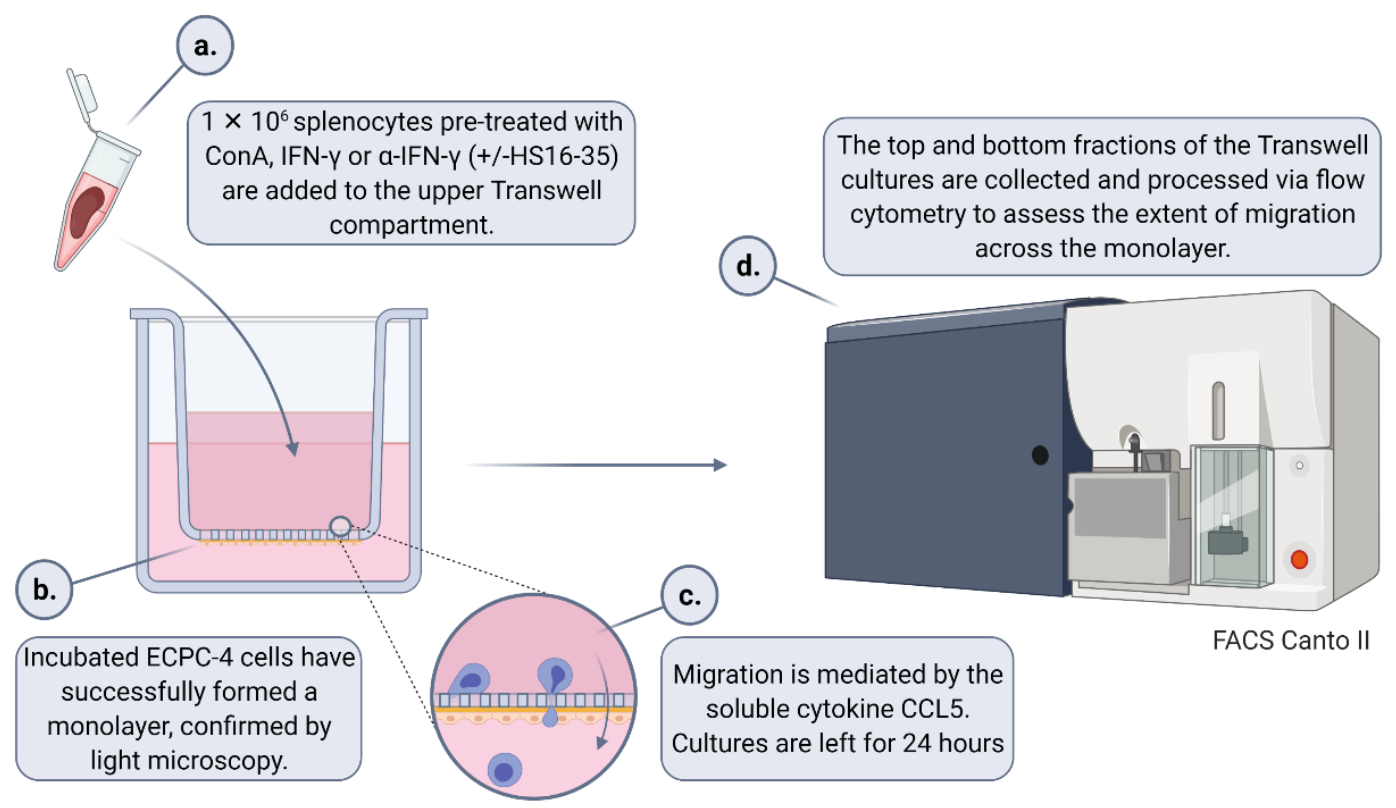

Figure 2.2: Simplified schematic showing how the transwell migration assay is run.

\subsection{Cytokine Stimulation: IFNy}

To simulate and inflammatory environment like that of EAE and MS, ECPC-4 cells were stimulated with IFN $\gamma$. IFN $\gamma$ promotes the upregulation of adhesion molecules on the surface of epithelial cells, mimicking the pathogenic conditions that occur in the brain of MS patients. 36 hours after seeding ECPC- 4 cells on Transwells as described I 2.4.I.2, IFN $\gamma$ was supplied in warm CTCM to the lower compartment of IFNY stimulated wells at a concentration of $20 \mathrm{U} / \mathrm{mL}$ for $24 \mathrm{~h}$ before the start of the migration assay.

\subsubsection{Splenocyte Migration}

This assay was used to assess the migration of splenocytes across an in vitro model of the choroid plexus. Splenocytes harvested and treated as described in 2.2.5 and 2.4.2, or untreated splenocytes were resuspended vigorously in their respective wells of the 96-well round bottom plate (In Vitro). Cells were then transferred to the upper compartments of prepared Transwell monolayer cultures, as detailed in 3.I. To ensure all cells were collected, $10 \mu \mathrm{L}$ warm CTCM was added to each well, the plate was vortexed for 15 seconds, and remaining media was transferred into the Transwells. The total duration of the migration phase of this experiment was $24 \mathrm{~h}$. 


\subsubsection{Chemokine Stimulation: CCL5}

The migration of splenocytes in the assay was mediated by CCL5 (RANTES) (Prospec). To the lower compartment of cytokine-stimulated wells, CCL5 was added at a concentration of $100 \mathrm{ng} / \mathrm{mL}$ in $600 \mu \mathrm{L}$ warm CTCM. To unstimulated wells, only fresh CTCM was added. The culture was incubated for 24 hours at $37^{\circ} \mathrm{C} / 5 \% \mathrm{CO}_{2} \mathrm{RH}$. To assess the capacity of migration, the fractions of the upper and lower wells were separated and prepared for Flow Cytometry. 


\subsection{Flow Cytometry}

To investigate the extent of migration across the monolayer, flow cytometry was performed after staining with a full panel of CD4-BV42I, CD45-BV5 I0, B220-AF488, CD8-PerCP-Cy5.5, CDI I b-PE-Cy7, CD62LAPC, CD44-PE and CD3-APC-Cy7.

\subsection{Antibody List}

Table 2: Materials for Flow Cytometry Staining

\begin{tabular}{|c|c|c|c|c|c|}
\hline Product & Label & Clone & Isotype & $\begin{array}{l}\text { Catalog } \\
\text { Number }\end{array}$ & Company \\
\hline $\begin{array}{l}\text { Anti-mouse/human } \\
\text { CD45R/B220 }\end{array}$ & AF488 & RA3-6B2 & Rat IgG2a, k & 103228 & BioLegend \\
\hline $\begin{array}{l}\text { Anti-mouse CD62L } \\
\text { Antibody }\end{array}$ & APC & MEL-I4 & Rat IgG2a, $\mathrm{k}$ & 104411 & BioLegend \\
\hline $\begin{array}{l}\text { Anti-mouse CDI Ic } \\
\text { Antibody }\end{array}$ & APC & N418 & $\begin{array}{c}\text { Armenian } \\
\text { Hamster IgG }\end{array}$ & 117310 & BioLegend \\
\hline $\begin{array}{l}\text { Anti-mouse CD3 } \\
\text { Antibody }\end{array}$ & APC/Cy7 & $17 A 2$ & Rat $\lg G 2 b, k$ & 100221 & BioLegend \\
\hline Anti-mouse CD4 & BV42I & GKI.5 & Rat $\lg G 2 b, k$ & 100438 & BioLegend \\
\hline Anti-mouse CD45 & BV5I0 & $30-\mathrm{FII}$ & Rat $\lg G 2 b, k$ & 103138 & BioLegend \\
\hline $\begin{array}{l}\text { Anti-mouse/human } \\
\text { CDIIb }\end{array}$ & PE & $\mathrm{MI} / 70$ & Rat $\lg G 2 b, k$ & 101208 & BioLegend \\
\hline Anti-mouse CD44 & PE & IM7 & Rat lgG2b, $k$ & 103007 & BioLegend \\
\hline Anti-mouse CD8a & PerCP/Cya5.5 & $53-6.71$ & Rat $\lg G 2 a, k$ & 100734 & BioLegend \\
\hline
\end{tabular}

\subsubsection{Preparation of Solvents}

\subsubsection{FACS buffer}

$2 \% \mathrm{FBS}$ and $0.1 \%$ IM sodium azide in IX dPBS.

\subsubsection{Fc Block}

Purified Rat Anti-Mouse CDI6/CD32 (BD) in diluted at 1:300 in FACS buffer.

\subsubsection{Compensation Beads}

One drop UltraComp eBeads ${ }^{\mathrm{TM}}$ Compensation Beads (ThermoFisher) in $100 \mu \mathrm{L}$ FACS buffer with I $\mu \mathrm{L}$ antibody working stock. 


\subsubsection{Fluorescence Minus One (FMO) Control}

$50 \mu \mathrm{L}$ full panel stain in FACS buffer, omitting desired FMO control antibody. For these experiments, FMO controls included B220, CD3, CDIIb, CD44 and CD62L.

\subsubsection{Full Panel Stain}

Table 3: Dilution of Antibodies in the full panel stain

\begin{tabular}{|l|c|}
\hline Product & Final Dilution in FACS Buffer \\
\hline Anti-mouse CD4 & $\mathrm{I}: 200$ \\
\hline Anti-mouse CD45 & $\mathrm{I}: 400$ \\
Anti-mouse/human CD45R/B220 & $\mathrm{I}: 400$ \\
Anti-mouse CD8a & $\mathrm{I}: 400$ \\
Anti-mouse/human CDI lb & $\mathrm{I}: 800$ \\
Anti-mouse CD62L Antibody & $\mathrm{I}: 400$ \\
Anti-mouse CD3 Antibody & $\mathrm{I}: 200$ \\
Anti-mouse CD44 & $\mathrm{I}: 400$ \\
\hline
\end{tabular}

\subsubsection{Flow Cytometry Protocol}

The full panel stain, Fc block and FMO controls were prepared and stored on ice, protected from light before the preparation of samples for Flow Cytometry. The upper and lower fractions of the Transwell culture were collected. To ensure all cells were collected, samples were vigorously re-suspended in their respective wells using a $1000 \mu \mathrm{L}$ pipette (Falcon) set to $2 / 3$ of the total volume of each sample. The underside of each Transwell was gently blasted with media to encourage partially migrated cells to detach. Medium containing cells from the upper well was plated directly into a round bottom 96-well plate (In Vitro). Media from the lower well were placed into $1.2 \mathrm{~mL}$ microcentrifuge tubes and centrifuged at $700 \mathrm{xg}$ for 5 minutes or until a visible pellet formed. $350 \mu \mathrm{L}$ supernatant was discarded, and the tubes were vortexed to resuspend cells in solution.

Cells were plated in a round bottom 96-well plate (In Vitro) in a checkerboard pattern. FACS buffer was added to each well to a final volume of $250 \mu \mathrm{L}$. The plate was centrifuged at $400 \mathrm{xg}$ for 4 minutes $\left(4^{\circ} \mathrm{C}\right)$. Supernatant was removed and plated in a flat-bottom 96 -well plate for utilization in ELISA assays. The 
plate was vortexed for 15 seconds to resuspend cells in the remnant media. $50 \mu \mathrm{L}$ Fc block was added to each well and the plate was incubated on ice, in the dark for 15 minutes. $150 \mu \mathrm{L}$ FACS buffer was then added to each well, the plate was centrifuged again at $400 \mathrm{xg}$ for 4 minutes $\left(4^{\circ} \mathrm{C}\right)$. Supernatant was discarded via gentle flicking. The plate was vortexed for 15 seconds to resuspend cells in the residual media. Each sample was re-suspended in $50 \mu \mathrm{L}$ full panel stain and placed on ice for 20 minutes, protected from light. Samples were centrifuged at $400 \mathrm{xg}$ for 4 minutes $\left(4^{\circ} \mathrm{C}\right)$ and re-suspended in $200 \mu \mathrm{L}$ FACS buffer. Samples were processed in the 96-well plate on a Canto II Flow Cytometer and BD FacsDiva Software. Results were analysed using Flowjo software (BD). 


\subsection{IFNp ELISA}

\subsection{Standards}

rmIFNY $(B D)$ standards were performed in duplicate rows of an ELISA plate, with the top standard set to $4 \mathrm{ng} / \mathrm{mL}$ in blocking buffer. 2-fold serial dilutions were performed to generate the standard curve, with two blank wells included for negative control.

\subsubsection{ELISA Protocol}

ELISA's were performed in accordance with the manufacturer's protocols, which will be summarised here (BD Biosciences). The capture antibody, rat anti-mIFN $\gamma(\mathrm{BD})$ was diluted in capture buffer (2.14.7) to a final dilution of $\mathrm{I} \mu \mathrm{g} / \mathrm{mL}$ and plated into a 96 -well ELISA plate at $50 \mu \mathrm{L} /$ well. The coated plates were incubated overnight at $4^{\circ} \mathrm{C}$, protected from light. The following day, plates were incubated with $100 \mu \mathrm{L}$ blocking solution (2.14.7) for two hours at room temperature. After this incubation, plates were washed 4 times in a wash buffer comprised of PBS with $0.05 \%$ Tween 20 (Sigma) and excess moisture from the wells was stamped onto a paper towel with force until wells appeared dry. Standards and samples were plated in $50 \mu \mathrm{L}$ aliquots and left for 120 minutes at room temperature. After this incubation, the plate was washed 4 times in the washing buffer again. The biotinylated detection antibody (biotin rat anti-mIFNy, BD) was diluted to $125 \mathrm{ng} / \mathrm{mL}$ in blocking buffer and added at a volume of $50 \mu \mathrm{L}$ to each well. The plate was again incubated at room temperature for one hour, then rinsed 6 times in the washing buffer and stamped to dry. Streptavidin horse-radish peroxidase (BD) was diluted in blocking buffer to I:2000 and added at $50 \mu \mathrm{L}$ to each well. The plate was incubated in the dark at room temperature for one hour, then washed eight times in the washing buffer. Room temperature tetramethyl benzidine (TMB) substrate $A$ and $B$ were thoroughly mixed in equal volumes and $100 \mu \mathrm{L}$ of this mixture was dispensed into each well. The plate was observed for colour change, and once a sufficient colour developed, stop solution (2.14.7) was added at a volume of $100 \mu \mathrm{L}$. The plate was read at $450 \mathrm{nM}$ on the EnSpire 2300 multilabel plate reader (PerkinElmer). A linear standard curve was generated using the values generated from the standard rows, and the concentration of IFN $\gamma$ in solution was quantified using the formula of the curve. 


\subsection{MTT Assay}

\subsection{Cell Specimens}

\subsection{I.I. Splenocytes}

Splenocytes were harvested from healthy mice as described in section 2.2 .5 and diluted to a single-cell suspension of $\mathrm{I} \times 10^{6}$ cells $/ \mathrm{mL}$ in warm CTCM. Cells were seeded into a 96-well round-bottom plate in $100 \mu \mathrm{L}$ aliquots, with an absolute cell number of $\mathrm{I} \times 10^{5}$ cells/well.

\subsubsection{ECPC-4 Cells}

ECPC-4 cells were cultured and prepared as described in 2.3.I into a single cell suspension of $I x$ $10^{5}$ cells $/ \mathrm{mL}$ in warm CTCM. Cells were seeded into a 96 -well round bottom plate in $100 \mu \mathrm{L}$ aliquots, with an absolute cell number of $\mathrm{I} \times 10^{4}$ per well.

\subsection{I.3. HSI6-35 Treatment}

The primary variable for this experiment was the concentration of HSI6-35. For the 48 hours prior to the addition of MTT solution splenocytes or ECPC-4 cells in a 96-well round bottom plate were treated with HSI6-35 at a concentration of $6 \mu \mathrm{g} / \mathrm{mL}, 60 \mu \mathrm{g} / \mathrm{mL}$ or $600 \mu \mathrm{g} / \mathrm{mL}$ in warm CTCM at $37^{\circ} \mathrm{C} / 5 \% \mathrm{CO}_{2} \mathrm{RH}$. $0 \mu \mathrm{g} / \mathrm{mL}$ was used as a control for each condition. Each treatment was performed in triplicate.

\subsubsection{Concanavalin A (ConA) Treatment}

For stimulation with ConA, cells were treated at I $\mu \mathrm{g} / \mathrm{mL}$ in a total of $200 \mu \mathrm{L}$, containing CTCM, +/- HSI635 and ConA. Splenocytes were stimulated for a total of $24 \mathrm{~h}$ at $37^{\circ} \mathrm{C} / 5 \% \mathrm{CO}_{2} \mathrm{RH}$. Media containing ConA was removed following centrifugation of the plate at $400 \mathrm{xg}$ for 4 minutes. Splenocytes were re-suspended in $200 \mu \mathrm{L}$ fresh CTCM without ConA and incubated for 24 hours prior to the addition of MTT solution.

\subsection{I.5. Interferon- $\gamma($ IFN $\gamma)$ Treatment}

For stimulation with IFN $\gamma$, cells were treated at $20 \mathrm{U} / \mathrm{mL}$ in a total of $200 \mu \mathrm{L}$, containing CTCM, +/- HSI635 and IFNy. Cells were stimulated with IFN $\gamma$ for a total of 48 hours at $37^{\circ} \mathrm{C} / 5 \% \mathrm{CO}_{2} \mathrm{RH}$. Supernatant containing IFN $\gamma$ was not replaced before the addition of MTT solution to the wells.

\subsubsection{MTT Assay Protocol}

The 96-well plate containing treated cells was centrifuged at $400 \mathrm{xg}$ for 4 minutes to settle cells at the bottom of the wells. $170 \mu \mathrm{L}$ of supernatant was removed and set aside for further analysis. Samples were then re-suspended in $50 \mu \mathrm{L}$ warm CTCM. To each well, $20 \mu \mathrm{L}$ sterile-filtered MTT solution (2.I4.8) was 
added. The samples were incubated for 120 minutes at $37^{\circ} \mathrm{C} / 5 \% \mathrm{CO}_{2} \mathrm{RH}$, protected from light. $50 \mu \mathrm{L} \mathrm{MTT}$ solubilizer (2.14.8) was added to each well. The plate was then incubated again at $37^{\circ} \mathrm{C} / 5 \% \mathrm{CO}_{2} \mathrm{RH}$, protected from light for a further 14 hours. The plate was read at $470 \mathrm{~nm}$ on a 2300 EnSpire ${ }^{\mathrm{TM}}$ Multilabel Plate Reader (PerkinElmer). 


\subsection{Evans Blue Permeability Assay}

\subsection{Solvents}

\subsection{I.I. Evans Blue Dye}

Evan's blue dye (Sigma-Aldrich) was diluted to a concentration of $0.67 \mathrm{mg} / \mathrm{mL}$ in warm CTCM. The dye was sterile filtered through a $0.22 \mu \mathrm{m}$ syringe filter unit (Millex-GP).

\subsubsection{Permeability Assay Protocol}

\subsubsection{Establishing a monolayer of ECPC-4 cells}

ECPC- 4 cells were seeded onto $8 \mu \mathrm{m}$ pore-size Transwells and cultured as described in methods section 2.4.I.2. The presence of an intact monolayer was verified using simple light microscopy.

\subsubsection{Treating the monolayer with HSI6-35}

120 minutes prior to starting the permeability assay, HSI6-35 was added at concentrations of $6 \mu \mathrm{g} / \mathrm{mL}$, $60 \mu \mathrm{g} / \mathrm{mL}$, or $600 \mu \mathrm{g} / \mathrm{mL}$ in warm CTCM to the lower compartment of each Transwell culture. A $0 \mu \mathrm{g} / \mathrm{mL}$ culture was used as a control, and all treatment conditions were performed in triplicate.

\subsubsection{Evans Blue Permeability Assessment}

Sterile Evan's Blue dye at $0.67 \mathrm{mg} / \mathrm{mL}$ was added in $200 \mu \mathrm{L}$ aliquots to the top compartment of each Transwell culture, taking care to dispense the dye slowly from the pipette tip. The cultures were incubated at $37^{\circ} \mathrm{C} / 5 \% \mathrm{CO}_{2} \mathrm{RH}$ for 90 minutes. Metal tweezers were used to remove the Transwells from each well of the 24-well plate and set aside for further analysis. The plate was swirled was gently swirled for 30 seconds to distribute the dye evenly, and $200 \mu \mathrm{L}$ aliquots were removed from each sample. In a 96 -well

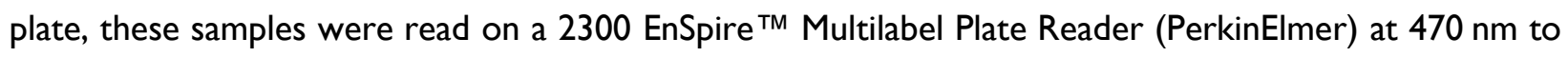
determine the extent of the dye that had crossed the monolayer. 


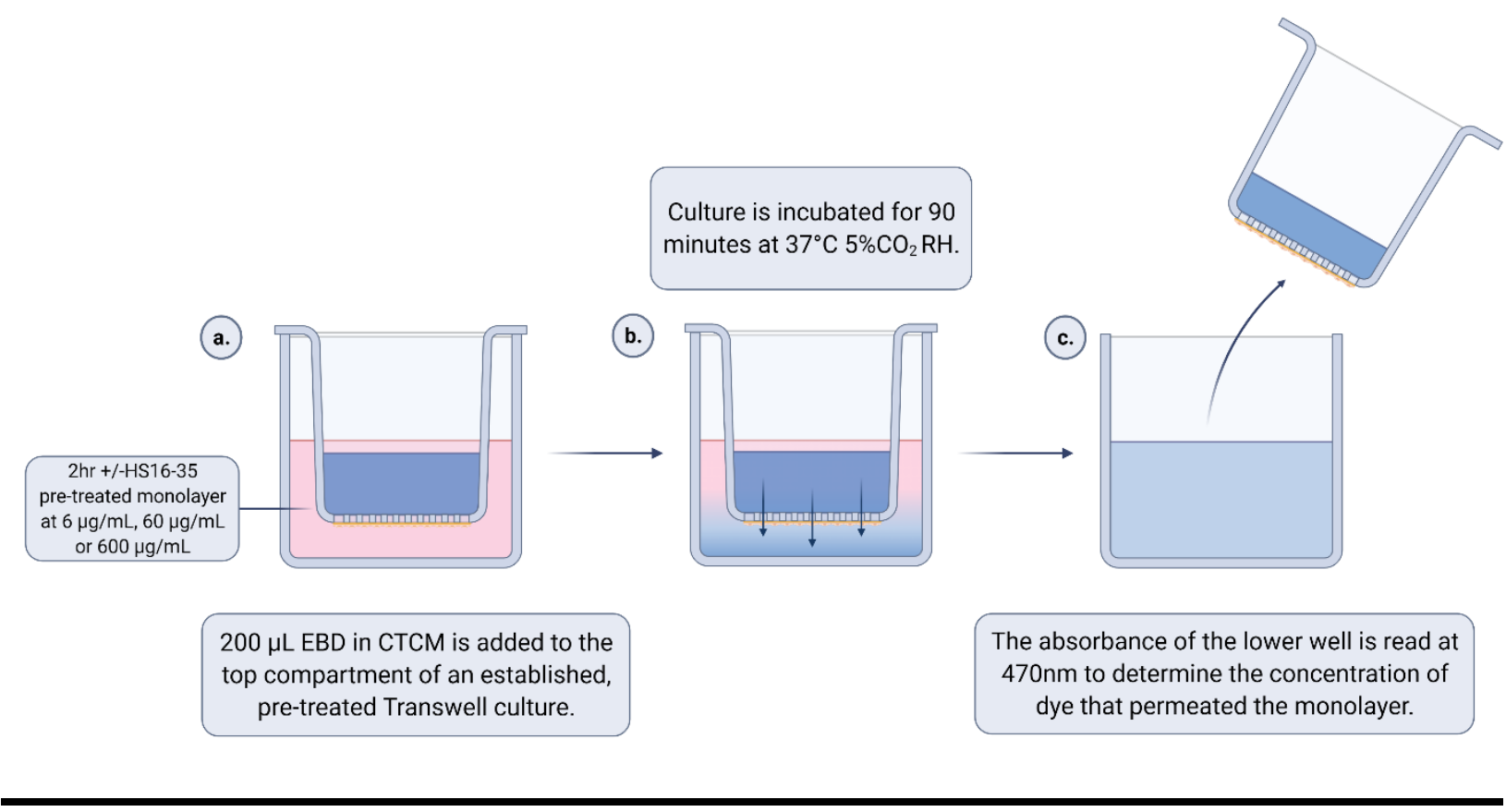

Figure 2.3: Simplified schematic showing how the EBD permeability assay is run. 


\section{I 0.LEGENDPlex ${ }^{\mathrm{TM}}$ Mouse Inflammation Panel}

This assay was conducted as according to the manufacturer's protocol which will be summarized below.

\subsection{Preparation of Tissue Culture Supernatant}

Live splenocyte cultures in a 96 -well plate were centrifuged at $400 \times g$ for 4 minutes. I $70 \mu \mathrm{L}$ supernatant from each well was removed and stored at $-20^{\circ} \mathrm{C}$ until required.

\subsubsection{Standard preparation}

The Mouse Inflammation Panel Standard Cocktail is supplied at 10,000 pg/mL when reconstituted in $250 \mu \mathrm{L}$ assay buffer (supplied in kit). For this assay, it was used undiluted as the top standard (C7). Serial I:4 dilutions of the cocktail were performed 6 times in succession to obtain a standard curve. One sample of assay buffer alone was used as a negative control ( $\mathrm{C} 0)$.

\subsubsection{Assay Procedure}

All reagents were warmed to room temperature before starting the assay. In a $96-$ well V-bottom plate, $25 \mu \mathrm{L}$ assay buffer was added to all wells. To the standard wells, $25 \mu \mathrm{L}$ standard was added, and in the test wells, $25 \mu \mathrm{L}$ cell-culture supernatant was added. Each standard and sample were performed in duplicate. The Mouse Inflammation Panel Premixed Beads were added in $8 \mu \mathrm{L}$ aliquots to each well, making the total volume $58 \mu \mathrm{L}$ in each well. The plate was sealed and covered in aluminium foil to protect from light. Samples were then shaken at $800 \mathrm{rpm}$ on a plate shaker for 120 minutes at room temperature. Following this, the plate was centrifuged at $250 \times \mathrm{xg}$ for 5 minutes to sediment the beads. The supernatant was discarded from the plate by forceful inversion. Each well was rinsed with $200 \mu \mathrm{L}$ wash buffer, and the plate was again shaken at $800 \mathrm{rpm}$ on a plate shaker. The centrifuge step was repeated, and supernatant discarded as previously described. $8 \mu \mathrm{L}$ detection antibodies were added to each well, and the plate was sealed and covered in aluminium foil to protect from light. The samples were shaken at $800 \mathrm{rpm}$ on a plate shaker for 60 minutes at room temperature. $8 \mu \mathrm{L}$ SA-PE was added to each well and the plate was shaken for a further 30 minutes at $800 \mathrm{rpm}$. The plate was centrifuged again, and supernatant discarded as described above. I50 $\mu \mathrm{L}$ Wash Buffer was added to each well and samples were resuspended by gentle pipetting. The samples were read using a Canto II flow cytometer on the same day, and results analysed using the LEGENDPlex software (v8.0) provided in the kit. 


\section{I I.Statistics}

Graphs and statistics for this thesis were generated using GraphPad Prism (v8.0). Some of the figures in this thesis were generated by combining experimental replicates from up to three experiments to account for inter-experimental variability and provide more statistical power. In experiments where two or more variables are being compared, we conducted a one-way analysis of variance (ANOVA) with Tukey's multiple comparison post-hoc test. For grouped analyses, (wherein two groups are compared) we conducted a two-way analysis of variance with Sisak's post-hoc multiple comparisons test. For all statistical analyses, significance was determined at $p<0.05(*=p<0.05$, ** $=p<0.05$, *** $=p<0.01$, **** $=p<$ $0.001)$. 



\section{Supplementary Methods}

\section{I 2. Establishing a Transwell Monolayer Culture}

These methods discuss the trialled conditions that were used to establish the transwell assay. These were tested and ultimately not used in the final methods, however formed an important part of this thesis, and will be discussed in the results section of chapter 3 .

\section{I2.I. Preparation of Transwells for Cell Culture}

\section{I2.I.I. Matrigel as a basement membrane}

Matrigel was thawed on ice overnight. All materials that Matrigel would contact (24-well plate, four $8 \mu \mathrm{m}$ Transwell@ inserts, pipette tips), were placed on ice for thirty minutes. Matrigel was diluted to a concentration of $3 \mathrm{mg} / \mathrm{mL}$ in ddH2O. $30 \mu \mathrm{L}$ diluted Matrigel was added to the inverted Transwell inserts, and the plate was incubated for 30 minutes at $37^{\circ} \mathrm{C} / 5 \% \mathrm{CO}_{2} \mathrm{RH}$ to allow the Matrigel to set. To ensure all excess Matrigel was removed, the inserts were washed twice with ice cold IX dPBS (Thermo Fisher). To avoid contamination, the Transwells were sterilised under a UV light, on ice for 30 minutes prior to seeding.

\subsubsection{Seeding ECPC-4 Cells onto Transwells (2 hours)}

ECPC-4 cells were passaged and re-suspended in a $50 \mathrm{~mL}$ conical centrifuge tube (Falcon) as described in the general methods. The concentration of cells was adjusted to $6.6 \times 10^{5}$ cells $/ \mathrm{mL}$ in warm CTCM. Cells were seeded at a volume of $75 \mu \mathrm{L}$ to the inverted mesh of the Transwell (absolute cell number $=5 \times 10^{4}$ ). To promote adherence, cells were incubated in this inverted orientation for $2 \mathrm{~h}$. At $2 \mathrm{~h}$, the Transwells were gently oriented back into an upright position using sterilized tweezers. To the lower compartment of the Transwell culture, $600 \mu \mathrm{L}$ complete growth media was added; enough to generously submerge the ECPC-4 monolayer.

\section{I 2.2. Transwell Migration Assay}

\section{I 2.2.I. Chemokine Stimulation: Embedded CCL5}

The migration of splenocytes in the assay was mediated by CCL5 (RANTES). Managing the chemokine gradient in experiment I, two variables were used; CCL5 embedded in low melting agarose or freefloating CCL5. This subsection describes how CCL5 in agarose was prepared, although ultimately this method was not used in the final Transwell model. 


\subsubsection{Low-melting agarose}

Under a laminar flow hood, of low-melting agarose was dissolved in DMEM at a concentration of 15 $\mathrm{mg} / \mathrm{mL}(\mathrm{I} .5 \%)$. The solution was heated in a microwave oven until bubbles appeared. The flask was swirled gently to ensure all agarose particles had been dissolved and left to cool.

\subsubsection{Dilution of soluble CCL5 chemokine}

CCL5 was provided as a stock solution of $1 \times 10^{5} \mathrm{ng} / \mathrm{mL}$ and diluted in the cooled agarose to a final concentration of $100 \mathrm{ng} / \mathrm{mL}$. The solution was resuspended gently to ensure the chemokine was evenly distributed before the agarose set.

\subsection{2.4. Seeding agarose into 24 well plates}

$200 \mu \mathrm{L}$ agarose/CCL5 solution was added into designated wells of a 24 -well plate. $200 \mu \mathrm{L}$ DMEM was added to each well containing the agarose, creating a 'slow release' of CCL5. The culture was incubated for 24 hours at $37^{\circ} \mathrm{C} / 5 \% \mathrm{CO}_{2} \mathrm{RH}$. To assess the capacity of migration, the fractions of the upper and lower wells were separated and prepared for Flow Cytometry. 


\section{I3.MTT Assay: CCL5 toxicity}

\subsection{Cell Specimens}

\section{I3.I.I. Splenocytes}

Splenocytes were harvested from three healthy mice as described in the 2.2.5, and diluted to a single-cell suspension of $\mathrm{I} \times 10^{6}$ cells $/ \mathrm{mL}$ in warm CTCM. Cells were seeded into a 96-well round-bottom plate in $100 \mu \mathrm{L}$ aliquots, with an absolute cell number of $\mathrm{I} \times 10^{5}$ cells/well.

\subsubsection{Treatment Conditions}

\subsubsection{CCL5 Treatment}

The primary variable for this experiment was the concentration of CCL5. Designated wells of a 96-well round-bottom plate were seeded with $100 \mu \mathrm{L}$ splenocyte suspension from each of the spleens as described in 2.2 .6 and stimulated with $100 \mu \mathrm{L} \mathrm{CCL5}$ at $100 \mathrm{ng} / \mathrm{mL}, 200 \mathrm{ng} / \mathrm{mL}, 400 \mathrm{ng} / \mathrm{mL}$ or $600 \mathrm{ng} / \mathrm{mL}$. A control condition of $0 \mathrm{ng} / \mathrm{mL}$ in $200 \mu \mathrm{L}$ warm CTCM was used for each spleen. The plate was then incubated for 24 hours at $37^{\circ} \mathrm{C} / 5 \% \mathrm{CO}_{2} \mathrm{RH}$.

\subsubsection{Starvation}

Our control included FCS starvation, wherein FCS was omitted from the CTCM.

\subsubsection{MTT Assay Protocol}

The 96-well plate containing treated cells was centrifuged at $400 \mathrm{xg}$ for 4 minutes to settle cells at the bottom of the wells. I $70 \mu \mathrm{L}$ of supernatant was removed and set aside for further analysis. Samples were then re-suspended in $50 \mu \mathrm{L}$ warm CTCM. To each well, $20 \mu \mathrm{L}$ sterile-filtered MTT solution (2.14.8) was added. The samples were incubated for 120 minutes at $37^{\circ} \mathrm{C} / 5 \% \mathrm{CO}_{2} \mathrm{RH}$, protected from light. $50 \mu \mathrm{L}$ MTT solubilizer (2.14.8) was added to each well. The plate was then incubated again at $37^{\circ} \mathrm{C} / 5 \% \mathrm{CO}_{2} \mathrm{RH}$, protected from light for a further 14 hours. The plate was read at $470 \mathrm{~nm}$ on a 2300 EnSpire ${ }^{\text {TM Multilabel }}$ Plate Reader (PerkinElmer). 


\section{I 4. Reagent Formulas}

All reagents used in tissue culture were sterile filtered through a $0.22 \mu \mathrm{m}$ syringe filter unit (Millex-GP Syringe Filter Unit, $0.22 \mu \mathrm{m}$, polyethersulfone, $33 \mathrm{~mm}$, gamma sterilized). All glassware used for tissue culture purpose was autoclaved prior to use.

\section{I4.I. Complete Growth Media (CGM)}

\begin{tabular}{|c|c|c|c|}
\hline Product & Composition & Company & Catalog Number \\
\hline Dulbecco's Modified Eagle Medium & $89 \%$ & Life Technologies & I I 995-073 \\
\hline Fetal Bovine Serum (FBS/FCS) & $10 \%$ & & \\
\hline Penicillin-Streptomycin & $1 \%$ & Life Technologies & $15140-122$ \\
\hline
\end{tabular}

\subsubsection{Complete Tissue Culture Media (CTCM)}

\begin{tabular}{|c|c|c|c|}
\hline Product & Composition & Company & Catalog Number \\
\hline Dulbecco's Modified Eagle Medium & $85.9 \%$ & Life Technologies & II995-073 \\
\hline Fetal Bovine Serum (FBS/FCS) & $10 \%$ & & \\
\hline Penicillin-Streptomycin & $1 \%$ & Life Technologies & $15 \mid 40-122$ \\
\hline HEPES & $1 \%$ & Thermo Fisher & 15630106 \\
\hline MEM Non-essential amino acids (NEAA) & $1 \%$ & In Vitro & $11 \mid 40050$ \\
\hline L-glutamine & $1 \%$ & Life Technologies & $25030-081$ \\
\hline Beta-mercaptoethanol & $0.1 \%$ & Life Technologies & $21985-023$ \\
\hline
\end{tabular}

\section{I4.3. Wash Buffer}

\begin{tabular}{|c|c|c|c|}
\hline Product & Composition & Company & Catalog Number \\
\hline Dulbecco's Modified Eagle Medium & $96 \%$ & Life Technologies & $11995-073$ \\
\hline Penicillin-Streptomycin & $1 \%$ & Life Technologies & $15 \mid 40-122$ \\
\hline HEPES & $3 \%$ & Thermo Fisher & 15630106 \\
\hline
\end{tabular}

2.14.4. Dulbecco's Phosphate-Buffered Saline

\begin{tabular}{|l|l|l|l|}
\hline Product & Composition & \multicolumn{1}{c|}{ Company } & Catalog Number \\
\hline$d P B S($ IOX), no calcium, no magnesium & $10 \%$ & Thermo Fisher & II $995-073$ \\
\hline$d d H_{2} \mathrm{O}$ & $90 \%$ & - & - \\
\hline
\end{tabular}


2.14.5. Fluorescence-Activated Cell Sorting (FACS) Buffer

\begin{tabular}{|c|c|c|c|}
\hline Product & Composition & Company & Catalog Number \\
\hline dPBS (IOX), no calcium, no magnesium & $9.8 \%$ & Thermo Fisher & II995-073 \\
\hline$d d H_{2} \mathrm{O}$ & $88.2 \%$ & - & - \\
\hline Fetal Calf Serum (FCS/FBS) & $2 \%$ & & \\
\hline
\end{tabular}

2.14.6. IOX Phosphate-Buffered Saline

\begin{tabular}{|l|l|}
\hline Product & Composition \\
\hline $\mathrm{NaCl}$ & $170 \mathrm{~g}$ \\
$\mathrm{Na} 2 \mathrm{HPO} 4 . \mathrm{I} 2 \mathrm{H} 2 \mathrm{O}$ & $62.32 \mathrm{~g}$ \\
$\mathrm{NaH} 2 \mathrm{PO} 4.2 \mathrm{H} 2 \mathrm{O}$ & $4.04 \mathrm{~g}$ \\
$\mathrm{ddH} \mathrm{H}_{2} \mathrm{O}$ & 2 litres \\
\hline
\end{tabular}

\section{I4.7. ELISA Buffers}

\begin{tabular}{|l|l|}
\hline Product & Composition \\
\hline Capture buffer & $0.1 \mathrm{M} \mathrm{Na}_{2} \mathrm{HPO}_{4}$ in $\mathrm{ddH}_{2} \mathrm{O}$, balanced to $\mathrm{pH} 9.0$. \\
Blocking buffer & $5 \% \mathrm{FCS}$ in IX PBS. \\
Stop solution & $0.18 \mathrm{M} \mathrm{H} 2 \mathrm{SO} 4$ in $\mathrm{ddH}_{2} \mathrm{O}$. \\
\hline
\end{tabular}

\subsubsection{MTT Buffers}

\begin{tabular}{|l|l|}
\hline Product & Composition \\
\hline MTT Solution & MTT at $5 \mathrm{mg} / \mathrm{mL}$ in PBS (sterile filtered) \\
MTT Solubiliser & $10 \%$ Sodium dodecyl sulfate (SDS) and $45 \%$ dimethylformamide \\
& (DMF) in $\mathrm{ddH}_{2} \mathrm{O} . \mathrm{pH}$ balance to 4.5 with $\mathrm{AcOH}$ \\
\hline
\end{tabular}

\subsubsection{Pertussis Toxin Buffer}

\begin{tabular}{|l|l|}
\hline Product & Composition \\
\hline Tris & $15 \mathrm{mM}$ \\
Sodium Chloride & $0.5 \mathrm{M}$ \\
\hline Triton $\mathrm{X}-100$ & $0.017 \%(\mathrm{v} / \mathrm{v})$ \\
\hline In $\mathrm{ddH}_{2} \mathrm{O}$ & \\
\hline
\end{tabular}



Chapter 3: Developing an in vitro model of the choroid plexus for the study of leukocyte trafficking 


\section{I. Introduction}

Migration is a fundamental cellular process for tissue development and homeostasis in multicellular organisms. Key biological processes rely on the capacity of cells to migrate in response to chemoattractants, such as conception, early embryo patterning and wound healing. The immune system relies on recruitment of lymphocytes from the lymph nodes to inflamed tissues to develop a protective immune response to pathogen invasion. ${ }^{147}$ Inversely, aberrant cellular migration can greatly contribute to disease pathology; for instance, in the development of tumour microenvironments and metastasis in cancer, or the invasion of autoimmune cells into an immune privileged site, in diseases such as MS.

The Transwell migration model and its associated migration assays are classical techniques that allow us to quantify the movement of various cell types through a microporous membrane. The model was first introduced by Dr. Stephen Boyden in 196I, who analysed the trafficking of leukocytes in response to chemotactic agents. ${ }^{48}$ Thus, the model is often referred to as the Boyden-Chamber assay. The applications of this model are broad, and vary greatly between papers, however the general premise is quite simple. Two media-filled wells are separated by a microporous polyester membrane. Cells are added to the upper well, and a chemokine or other stimuli is applied to the lower well. The micropores of the Transwell membrane are selected so that cells must actively rearrange their cytoskeleton to migrate through the membrane. ${ }^{147}$ This negates the idea of passive migration, in which cells simply fall through the membrane pores due to gravity, which is not considered true migration. Some migration assays, including the one presented in this thesis include a monolayer of cells across the membrane, which mimic a barrier structure. We incorporated epithelial ChP cells in this model, as cells entering the CSF through the ChP must navigate across the epithelium, a highly selective structure.

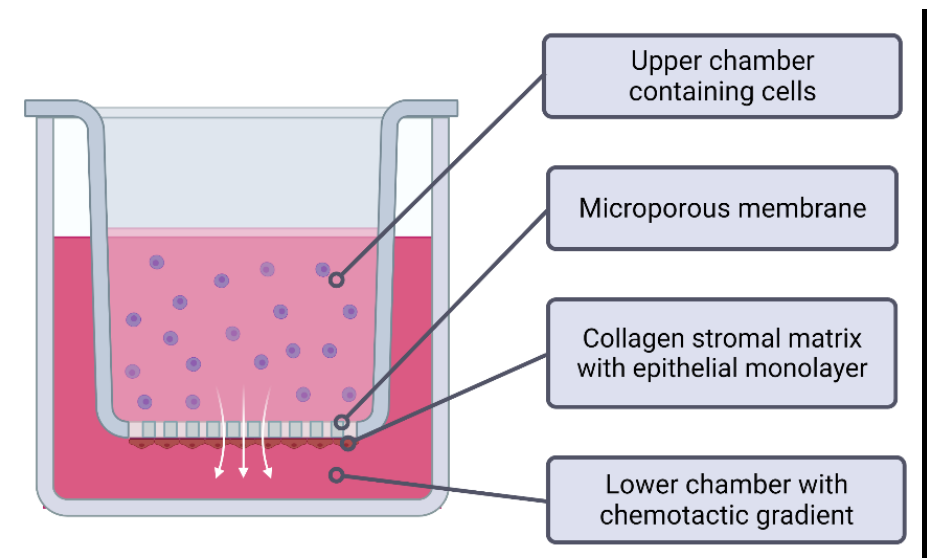

Figure 3.I: Schematic showing a Transwell model. The upper compartment of the Transwell cultures was considered the 'Peripheral Blood', and the lower chamber was considered the 'Cerebral Spinal Fluid', where a chemoattractant was added. A collagen coating was applied to the outer edge of the membrane to act as the ChP stromal matrix. Epithelial ChP cells were seeded onto the collagen layer. 


\subsection{Aims}

This chapter explores the processes and protocols that were used to develop a functional model of the $\mathrm{ChP}$ in vitro. Previous literature has shown that in vitro transwell models can be helpful to characterise the cell and molecular mechanisms that mediate pathogenic migration of lymphocytes into the CNS. Certainly, the ChP's anatomical location makes it a difficult structure to study in vivo, so there is a definitive need in the field. To faithfully reproduce the ChP's barrier properties and in vivo features, we conducted an extensive review of the current literature to select the most appropriate model to begin with. Our initial model was based upon Monnot and Zheng's (2013) paper, which introduced a protocol for a transwell model of the ChP using primary rat-derived ChP cells. 149 Throughout the course of this thesis, we adapted aspects of this model where we saw opportunity to more accurately mimic characteristics and functionality of the ChP.

We investigate the use of chemo-attractants and proinflammatory molecules to create a platform for inflammatory and pathogenic migration, similar to that of MS. This section was also crucial to develop a functional model to test our heparan-sulfate mimetic 'HSI6-35', which has a proposed mechanism of action that includes inhibition of migration into the CNS.

It is important to note that many of the experiments in this chapter were completed only once, that these assays served as 'checkpoint experiments' in which we confirmed whether techniques that were adapted from other papers were functional for use in this thesis.

\subsection{Specific Aims}

I. To develop an in vitro model of the choroid plexus.

2. To demonstrate that this experimental approach models migration across an epithelial monolayer in both homeostatic and inflammatory conditions. 


\subsection{Results}

\subsection{CCL5 is a non-toxic chemokine to splenocytes}

The CCL5 (RANTES) receptor (CCR5) has been shown to be significantly upregulated on $\mathrm{CD}^{+}, \mathrm{CD} 8^{+}$ and CDI9+ lymphocytes in active lesions of MS patients. 150 It's cognate ligand, CCL5 is a potent T cell chemoattractant that facilitates the homing process of $T_{H} l$ cells to lesion sites through the choroid plexus. ${ }^{39}$ Animal models of MS have shown that CCL5 can be upregulated by I46-fold in the ChP stroma and capillaries during active disease. ${ }^{151}$ Previous work by this group has shown that clozapine-treated mice have delayed onset EAE and reduced disease severity versus vehicle treated controls, in part due to reduced infiltration of lymphocytes into the CNS. Clozapine significantly downregulates the expression of CCL5 and CCL2 in the CNS compartment, implicating these chemokines as responsible for the early infiltration of autoreactive lymphocytes in EAE.152 In our model of the ChP, we selected CCL5 as a chemoattractant, based upon other migration models that had found CCL5 to be the most potent inducer of migration versus a panel of other chemokines in cells isolated from MS patients. ${ }^{153}$

Previous literature using CCL5 as an in vitro chemokine has ranged in dose from $20 \mathrm{ng} / \mathrm{mL}$ to $600 \mathrm{ng} / \mathrm{mL}$ to $600 \mathrm{ng} / \mathrm{mL}$. ${ }^{154-157}$ To gauge the most effective yet cost-effective dosing regimen of CCL5 (Prospec) on leukocytes, we assessed the viability of isolated splenocytes from C57BL/6] mice in response to 24h stimulation with control CTCM media, or media supplemented with increasing concentrations of CCL5 (Figure 3.2). Stimulation with $100 \mathrm{ng} / \mathrm{mL}$ significantly increased viability from control across three experiments by up to $150 \%$. It appears that both 200 and $400 \mathrm{ng} / \mathrm{ml}$ treatments also trended towards an increased viability, however these results are not significant. In contrast to these results, $600 \mathrm{ng} / \mathrm{mL}$ of CCL5 began to have a cytotoxic effect on splenocyte integrity, as viability was below control values, and this reduced viability was significant compared to the $100 \mathrm{ng} / \mathrm{mL}$ treatment. From these findings, we selected the dose with greatest increase in viability but lowest cost (i.e. $100 \mathrm{ng} / \mathrm{mL}$ ) for our transwell model. 


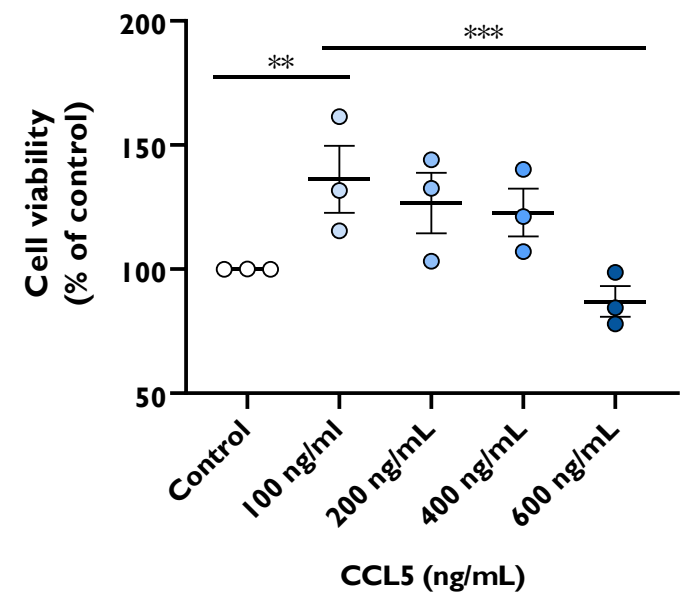

Figure 3.2: High concentrations of CCL5 decrease splenocyte viability. Shown are viability scores as a percentage of untreated control with mean \pm SEM. Cytokine CCL5 is nontoxic to splenocyte cultures following 24h stimulation at 100, 200 and $400 \mathrm{ng} / \mathrm{mL}$ in CTCM ( $\mathrm{n}=3$ spleens, with 2 duplicates. Results were analysed using an ordinary two-way ANOVA $(*=p<0.05, * *=p<0.05, * * *=p<0.01)$. 


\subsubsection{Matrigel is not a suitable stromal matrix component}

The choroid plexus is a dynamic tissue structure, with three core components that are adaptable to an in vitro model. (I) the tight barrier of epithelial cells, with their apical side facing the CSF, (2) the underlying stromal matrix which houses a resident population of immune cells, and (3) the layer of fenestrated endothelium with direct contact to the peripheral blood. The $8 \mu \mathrm{m}$ diameter micropores of the polyester (PET)-membrane mimic the leaky endothelium, which requires extravasating lymphocytes to actively rearrange their actin cytoskeleton to migrate across. Selection of an appropriate stromal matrix for this model requires immune cells to be able to migrate successfully through it. The matrix must also provide essential directional cues to the cultured epithelial monolayer as to which side is apical. Mature ChP epithelium is apically enriched with microvilli and transport machinery, whereas the nuclei are basally located. 158 Tight junction molecules form between cells and adhesion molecules are expressed on the apical face and alongside barrier molecules. Matrigel (Corning), is a common basement membrane component for the use of cell invasion assays. For cells to effectively navigate through an extracellular matrix in vivo or Matrigel in vitro, they must secrete appropriate matrix modifying proteases, such as MMP's. 159

We assessed the capacity of leukocytes isolated from the spleen of C57BL/6] mice to migrate through an $8 \mu \mathrm{m}$ micropore transwell (Corning) coated with Matrigel solution (Figure 3.4). Matrigel was diluted to $3 \mathrm{mg} / \mathrm{mL}$ and aliquoted at $30 \mu \mathrm{L} /$ well, then set at $37^{\circ} \mathrm{C}$ for 30 minutes before isolated splenocytes were added to the upper chamber of the transwell. After 24 hours, we isolated the upper and lower fractions of the transwell culture and used flow cytometry to assess the extent of migration across the basement membrane. Results from this assay showed that Matrigel almost completely inhibited the trafficking of CD45+ leukocytes across the transwell, versus the transwell alone, regardless of stimulation with CCL5 (Figure 3.4). This shows that cells did not express the appropriate matrix-modifying proteases associated with an invasion phenotype. 

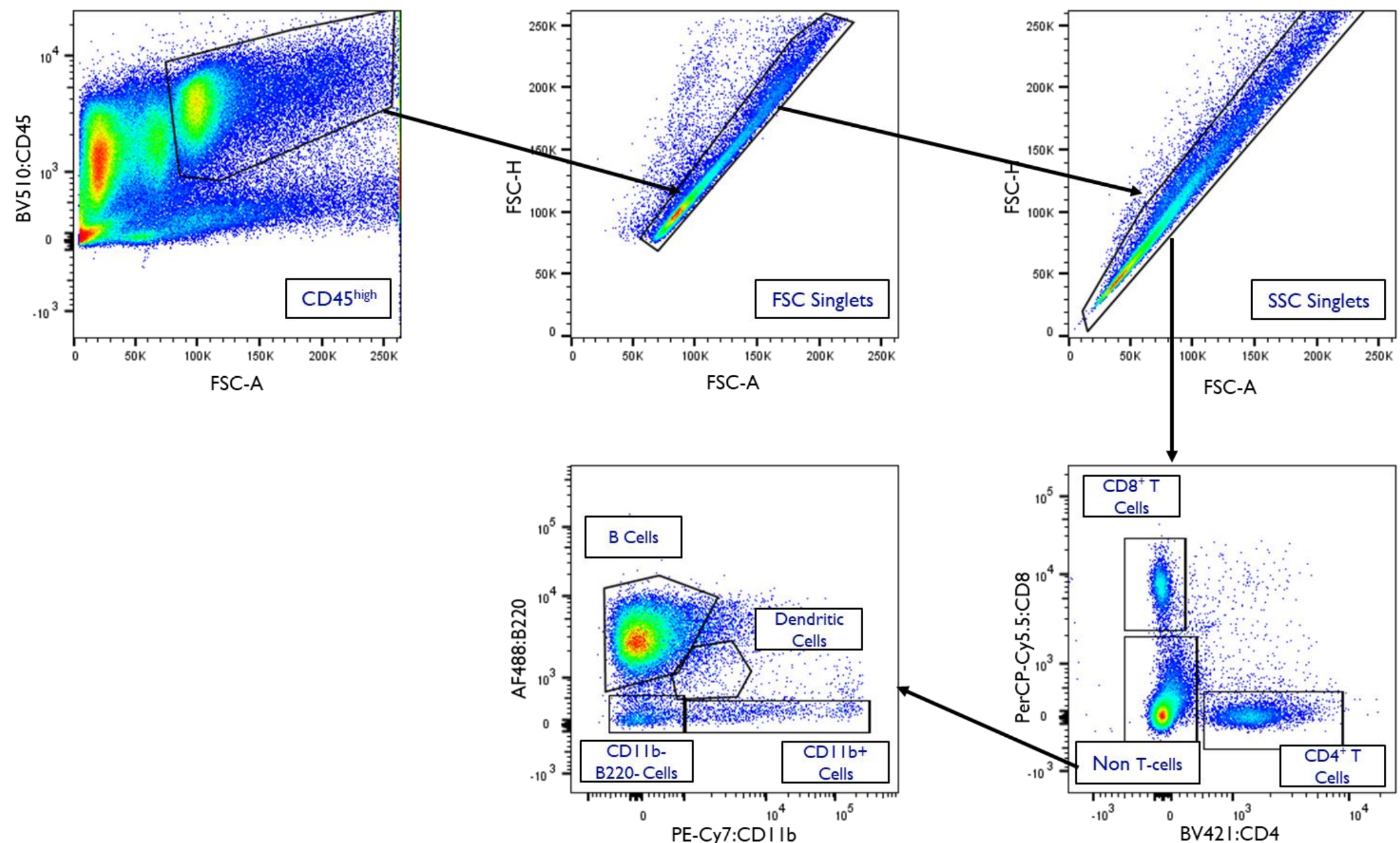

Figure 3.3: The gating strategy used to analyse flow cytometry data from the migration experiments in Chapter 3. Our gating strategy for flow cytometry in chapter 4. All flow cytometry data was assessed using Flowjo v10. Initially, CD45high lymphocytes were gated out, then gated to exclude doublets. All CD45high singlets were placed on a CD4 vs CD8 plot and gated for CD4 ${ }^{+}$and $C D 8^{+}$expression. Cells that were non- $T$ cells were then gated based on B220 vs CDI lb expression, with B220 high CD I lb- as B cells, B220 int CDI l lb+ as dendritic cells and B220-CDI l b ${ }^{+}$as myeloid cells. 


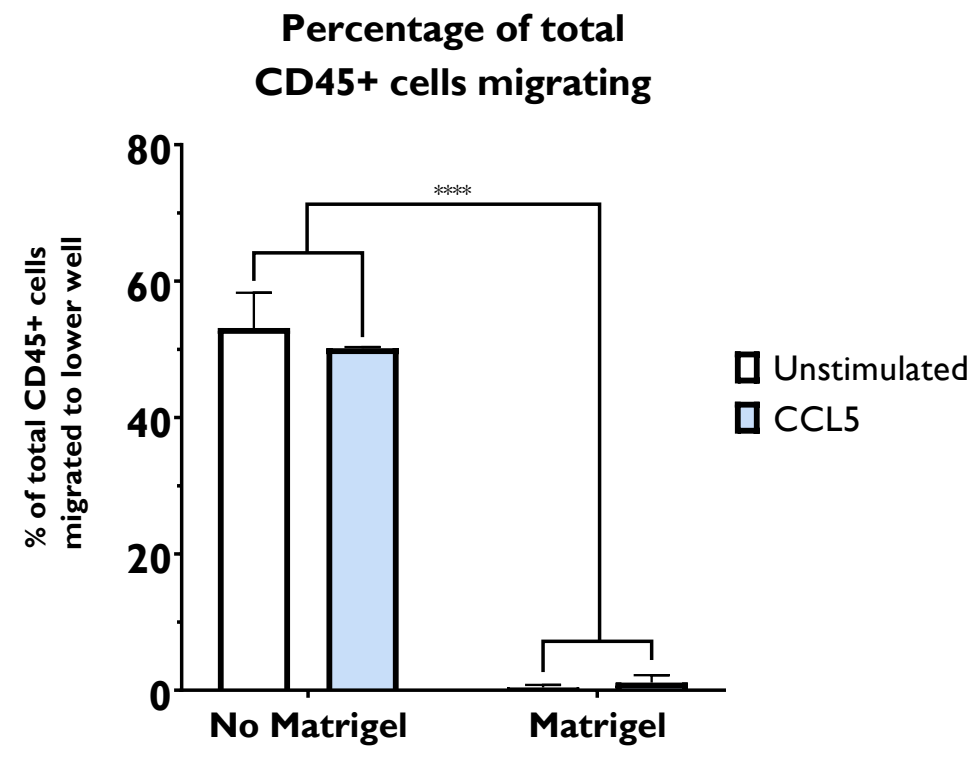

Figure 3.4: Matrigel is not a suitable basement membrane component for studying leukocyte trafficking. Shown are the means \pm SEM of the percentage of CD45+ splenocytes that migrated through a Transwell culture either without a basement membrane or with Matrigel as a basement membrane component $(n=2)$. Matrigel significantly reduced the number of migrating leukocytes through the Transwell culture. Results were analysed using an ordinary two-way ANOVA, with significance determined at $p<0.05(*=p<0.05$, ** $=p<0.05$, *** $=p<0.01$, **** $=p<0.001)$. 


\subsubsection{ECPC-4 cells are capable of forming a functional monolayer}

The epithelial monolayer of the $\mathrm{ChP}$ is arguably the most important factor in forming an effective bloodCSF barrier and maintaining the immune privilege of the CNS. We investigated the capability of the commercially available epithelial choroid plexus cell line 'ECPC-4' to form a selective, functional monolayer for the study of in vitro trafficking across the ChP. ECPC-4 cells have been reported to maintain the characteristics of $\mathrm{ChP}$, with expression of specific molecules such as transthyretin and $\alpha_{2}$-macroglobulin in long-term culture. 160 On Transwell inserts coated with $0.01 \%$ rat tail collagen (type II), ECPC-4 cells formed a confluent monolayer less than 24 hours after seeding at $1.66 \times 10^{5} \mathrm{cells} / \mathrm{cm}^{2}$ (Figure 3.5a, 3.5b). Even with extended culture, we saw that ECPC-4 cells were capable of forming contact-inhibited monolayers on transwell inserts.

To measure the functionality of our ECPC-4 monolayer, we adapted an Evans blue dye assay for in vitro use, allowing us to test the permeability of a Transwell culture treated with dye in a more rudimentary sense. Evans blue is classically used in vivo to assess the barrier function of the BBB in healthy animals versus EAE. The dye easily passes through leaky, inflamed endothelium but not healthy vasculature. In vitro, we utilized the model to assess the barrier capacity of the epithelial monolayer. We saw that ECPC-4 cells that had formed a confluent monolayer were effective in preventing dye from diffusing into the lower chamber versus a collagen coated transwell alone, indicating the proper formation of tight barriers in our in vitro culture (Figure 3.5c). In a checkpoint experiment, we assessed whether isolated splenocytes from C57BL/6] mice were capable of migrating across an ECPC-4 monolayer. At 24 hours after splenocytes were added to the upper transwell chamber, we saw that CD45+ leukocytes were capable of migrating through the monolayer, however at a lower frequency than if there was no epithelium (Figure 3.5d). This is indicative that the ECPC-4 monolayer is governing selective migration of CD45+ leukocytes. In these cultures, it appears that CCL5 stimulation has a greater impact on cell migration than the transwell alone. We hypothesize that when no epithelial monolayer is present, CCL5 freely diffuses into the upper chamber of the transwell culture, and cells do not follow a chemotactic gradient into the lower well. Nonpermeable tight junction molecules between ECPC-4 cells in our transwell culture likely inhibit the paracellular movement of CCL5 in large volumes, maintaining a chemotactic CCL5 gradient that CD45+ cells are responsive to. 
a.

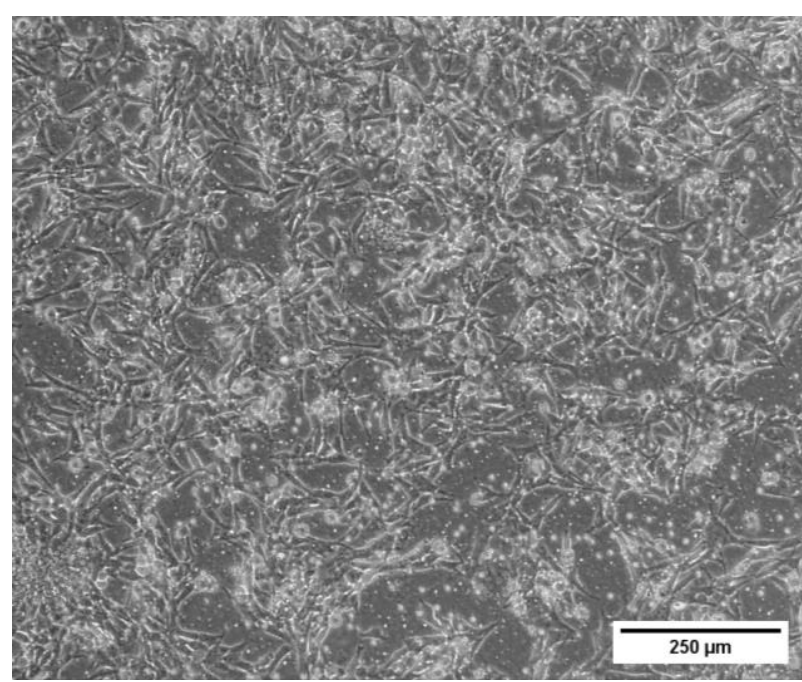

b.

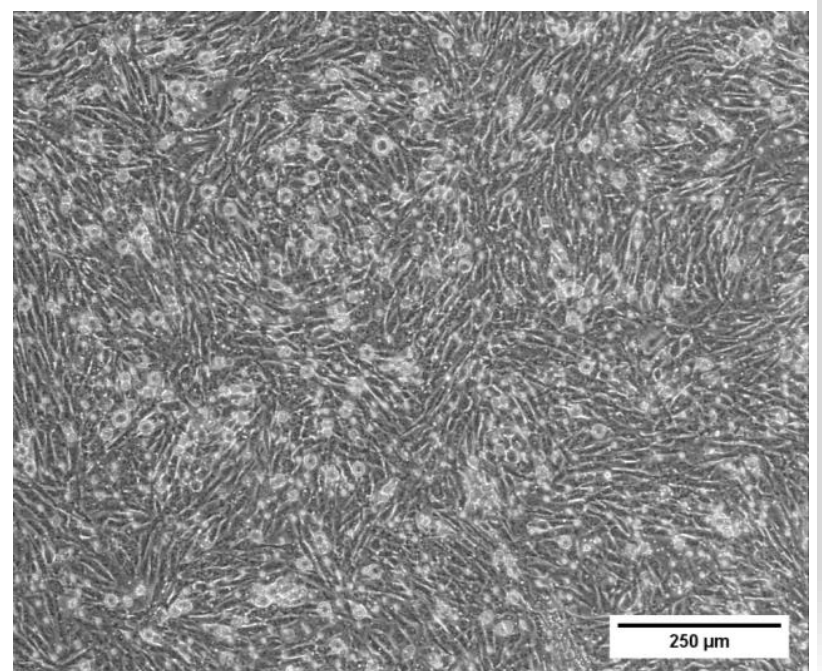

C. EBD Permeation through Transwell with ECPC-4 epithelial layer vs Collagen alone

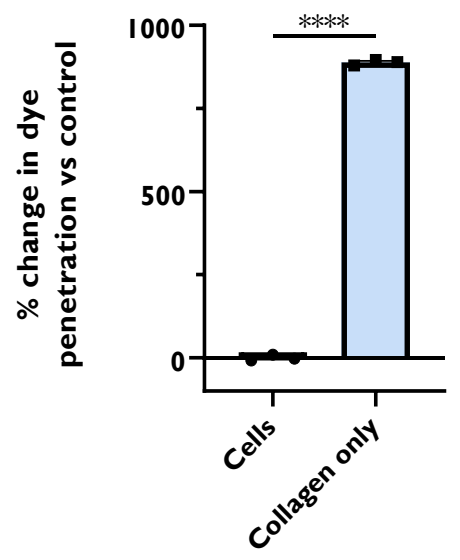

d.

Effect of ECPC-4 monolayer on CD45+ migration

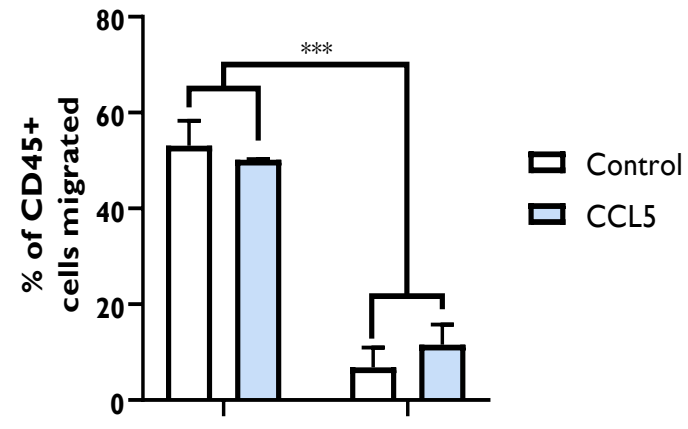

(-) ECPC-4 (+) ECPC-4

Figure 3.5: ECPC-4 cells are capable of forming an intact but permeable barrier on Transwells after 24h (a). Shown are Epithelial Choroid Plexus Cells (ECPC-4) I $2 \mathrm{~h}$ after seeding onto a Transwell at $1.66 \times 10^{5}$ cells $/ \mathrm{cm}^{2}$. (b) The same Transwell culture at $24 \mathrm{~h}$ post-seeding, now formed a confluent monolayer culture. The error bar represents $250 \mu \mathrm{m}$. (c) Evan's blue dye was added to the top compartment of Transwell cultures \pm epithelial layer. Shown is the extent of dye penetration into the lower well versus a control culture without dye means \pm SEM. When only basement membrane matrix (collagen) was present, there was a significant increase in the concentration of dye that permeated into the lower well versus with an intact epithelial monolayer $(n=3)$. Results were analysed using an unpaired $t$ test with significance set at $p<0.05(*=p<0.05$, ** $=p<0.05$, *** $=p<0.01$, **** $=p<0.00 \mathrm{I})$. (d) Shown are the means \pm SEM comparing CD45+ migration across a transwell culture lacking an epithelial monolayer or with an established ECPC-4 monolayer $(n=2)$. ECPC-4 cells significantly reduced migration into the lower well versus control. Results were analysed using an ordinary two-way ANOVA, with significance determined at $p<0.05(*=p<0.05$, ** $=p<0.05$, *** $=$ $\mathrm{p}<0.01$, **** $=\mathrm{p}<0.00 \mathrm{I})$. 


\subsubsection{Inflammatory migration can be induced with Concanavalin A stimulation}

One of the specific aims for adapting this model for the study of leukocyte trafficking across the ChP was to show that this model can mimic inflammatory trafficking in the context of MS. It is clear that the neuroinflammation in models such as the EAE model alters the transcriptome of the ChP from healthy controls, heavily upregulating the expression of adhesion molecules, $T$ cell activation markers, such as CDI54 and proinflammatory cytokines and chemokines. ${ }^{151,161,162}$

Because MS and EAE are primarily T-cell mediated diseases, we stimulated isolated splenocytes from healthy C57BL/6] mice with Concanavalin A for 24 hours prior to putting them in a Transwell culture. ConA is a lectin extracted from Canavalia ensiformis (jack beans), that irreversibly binds $\alpha-D$-mannosyl and $\alpha-D$-glucosyl residues to activate the immune system, induce cytokine production and the recruitment of lymphocytes. ${ }^{163}$ ConA acts as a potent $T$ cell mitogen by inducing cross linking of the TCR, leading to $T$ cell activation and proliferation in an antigen-independent manner. ${ }^{164}$

Because ConA has previously been shown to induce clustering of lymphocytes, we could not add our cell cultures stimulated with ConA directly into the Transwells and assume the clumped lymphocytes would fit through the pores. 165 After 24 hours of ConA stimulation, the media containing ConA was aspirated from the plate and splenocytes were resuspended in fresh media to reverse any agglutination of lymphocytes in the Transwell culture. The results from two independent experiments showed that splenocytes stimulated with ConA prior to the Transwell migration assay exhibited a significant increase in the number of cells that migrated across the ECPC-4 monolayer into the lower well versus the mediaalone control (Figure 3.6). This suggests that ConA is an excellent pro-inflammatory stimulus for mimicking inflammatory trafficking across the ChP.

To further examine the effects of ConA stimulation on the Transwell migration model, we utilized flow cytometry to look at a breakdown of the individual cell types penetrating the $\mathrm{ChP}$ monolayer. As expected, we observed a significant increase in the number of $\mathrm{CD} 4^{+}$and $\mathrm{CD} 8^{+} \mathrm{T}$ cells migrating across the Transwell culture in response to ConA versus control (Figure 3.7a). These cells appeared to become more sensitized to CCL5 when ConA-stimulated, with a trend toward higher cell counts in the +CCL5 condition versus -CCL5. In contrast to T cells, B cells, B220int CDI l b+ dendritic cells and CDI I b+ myeloid cells were not greatly affected by ConA stimulation, as there was no observable increase in cell number migration across the monolayer. (Figure 3.7) An appendix of experimental replicates can be found at the end of this chapter. 


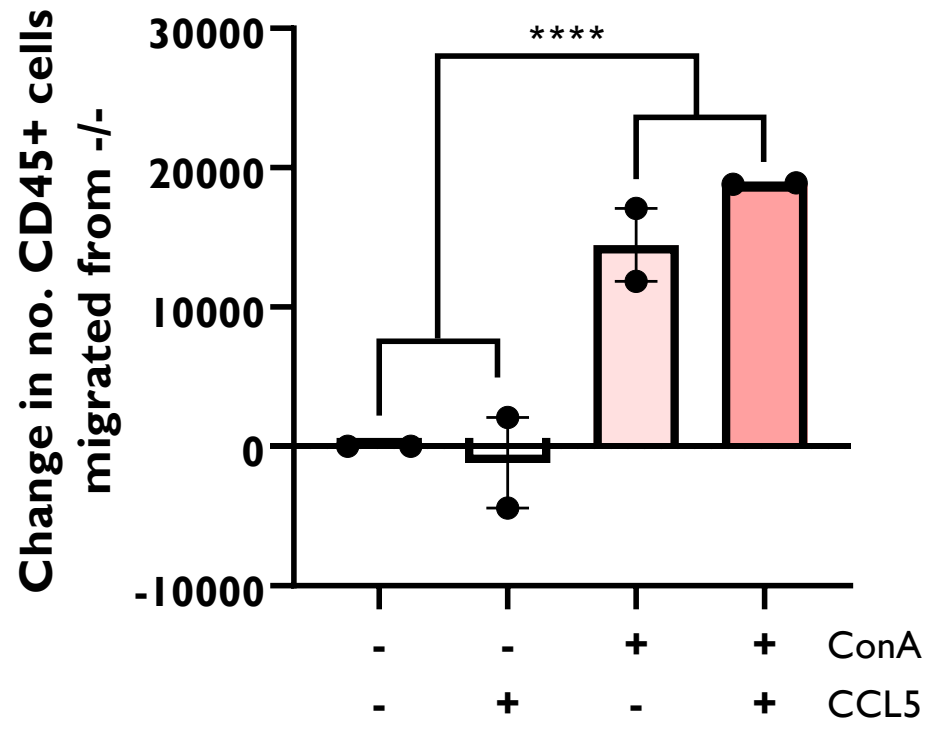

Figure 3.6: ConA induces inflammatory migration across the ECPC-4 monolayer. (a) Shown are the means \pm SEM from 2 experiments $(n=6)$ in which ConA significantly increased the number of migrating CD45+ healthy splenocytes across an epithelial monolayer in vitro. Analysis was conducted using an ordinary 2-way ANOVA, with significance at $p<0.05(*=p<0.05$, ** $=p<0.05$, $* * *=p<0.01, * * * *=p<0.001)$. 
a.

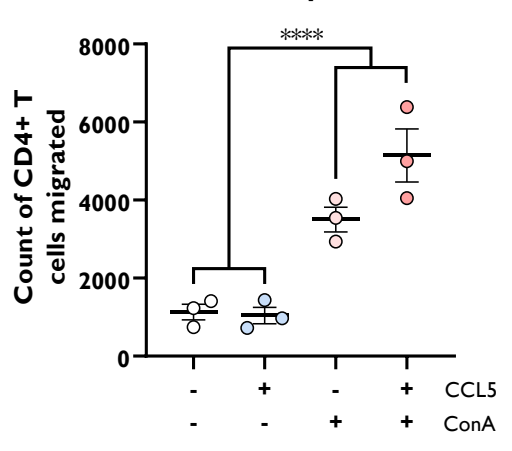

Treatment Condition
CD8+ T Cell response to ConA

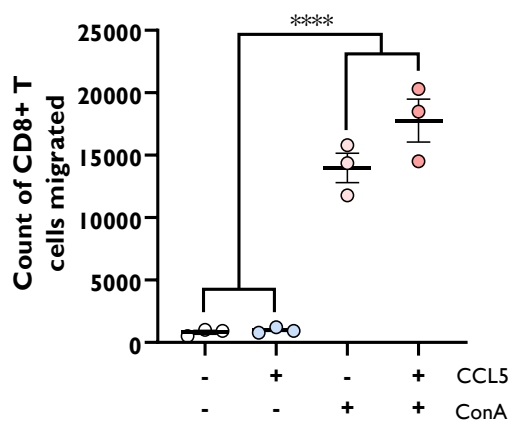

Treatment Condition

b.

B Cell response to ConA

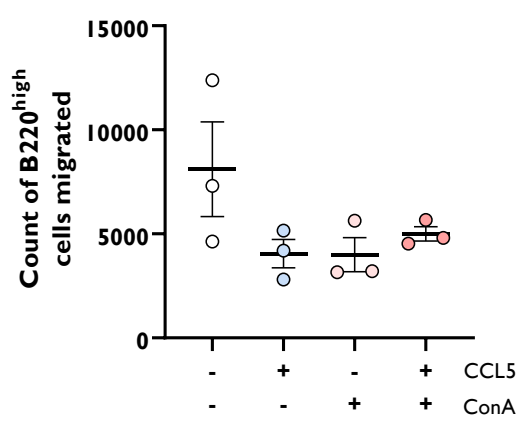

Treatment Condition
Dendritic Cell Response to ConA

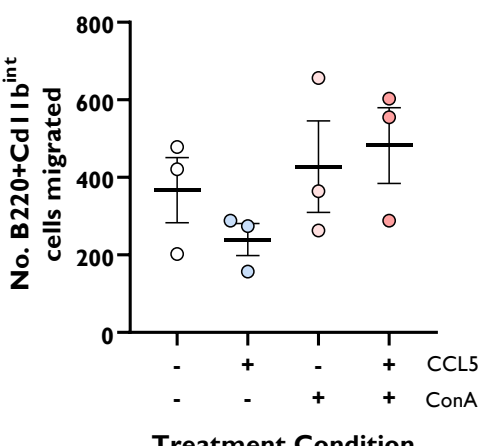

Treatment Condition
Myeloid Cell Response to ConA

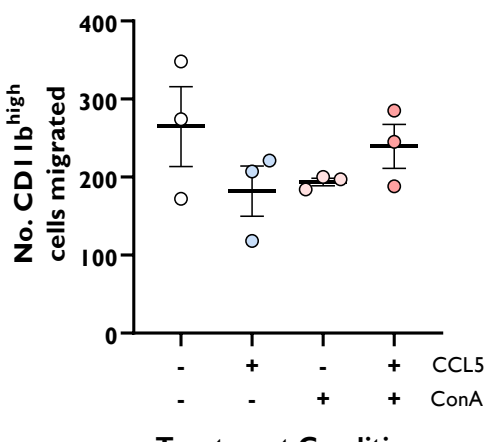

Treatment Condition

Figure 3.7: ConA elicits a greater migratory response from $T$ cells: (a) Shown are technical replicates from one experiment $(n=3)$. There was a clear increase in CD4+ T cell migration across the monolayer in response to $24 \mathrm{~h}$ ConA stimulation. CD4+ $T$ cells stimulated with ConA were more sensitive to and showed a stronger chemo-attractive response to CCL5. Analysis on these samples were performed using an ordinary two-way ANOVA with significance determined at $p<0.05$. CD8+ $T$ cells in this culture showed a similar pattern to CD4+ $T$ cells, with a ConA-dependent increase in migration and sensitization to CCL5 chemokine stimulation. Data was analysed with an ordinary twoway ANOVA with significance determined at $p<0.05$. ( $*=p<0.05, * *=p<0.05$, *** $=p<0.01$, **** $=p<0.00 \mathrm{I})$. (b). By contrast, in the same splenocyte culture, there is no observable change in $B$ cell, Dendritic Cell or general myeloid cell migration across an epithelial monolayer after $24 \mathrm{~h}$ in co-culture. 


\subsubsection{Inflammatory migration induced with ConA is partially IF $N_{Y}$ dependent}

Because IFN $Y$ plays such an essential role in mediating the autoimmune response in MS and EAE, alongside dysfunctional immune responses at the $\mathrm{ChP}$ in other disorders, we suggested that IFN $\gamma$ produced as a result of ConA stimulation was the key cytokine responsible for enhanced migration across the ECPC-4 monolayer in our model.166,167 To confirm this, we proposed that treatment with an anti-IFN $y$ antibody following ConA stimulation would reduce the overall number of cells migrating across the monolayer if IFN $\gamma$ was the primary cytokine responsible for enhancing migration in our model. Interestingly, in murine splenocyte cultures that were stimulated with ConA for 24 hours, which were then subsequently treated with an $\alpha \mathrm{IFN} \gamma$ antibody for 24 hours, there was a trend towards significant reduction in the number of CD45 ${ }^{+}$lymphocytes that migrated to the lower chamber (Figure 3.8a). However, the reduction in cell count for these $\alpha \mathrm{IFN} \gamma$ treated conditions was still significantly above the media-alone control, indicating that IFNY plays a large role but is not fully responsible for inflammatory migration across the ECPC-4 monolayer (Figure 3.8a).

Analysis of the supernatant from these experiments revealed that ConA induced a strong IFN $\gamma$ response flooding into the supernatant, which could be completely ablated by alFN $\gamma$ treatment (Figure $\mathbf{3 . 8 b}$ ). Together, these results suggest that IFN $\gamma$ is in-part responsible for increased migration of splenocytes across the epithelial monolayer, but other factors, including cytokine production and TCR activation through ConA are required for inflammatory migration similar to EAE or MS. 
a.

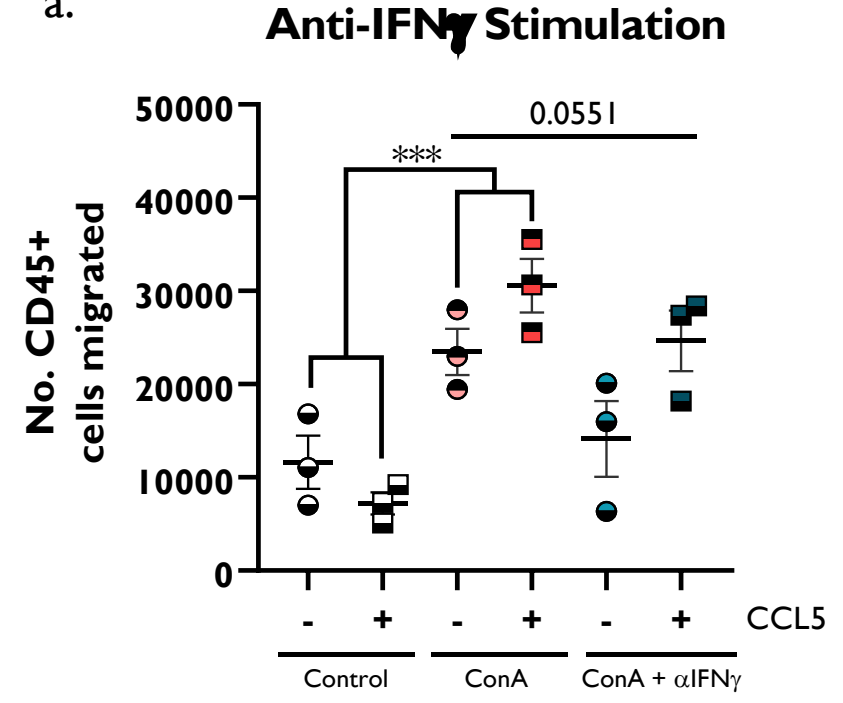

Treatment Condition b.

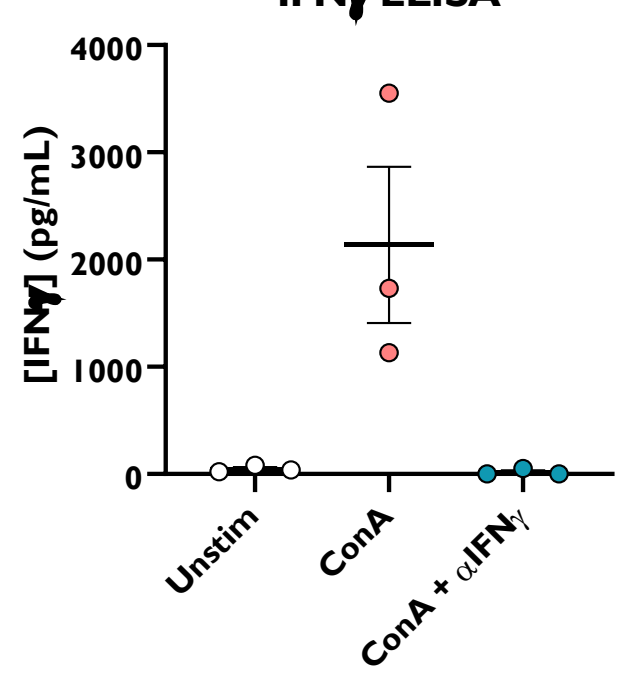

Treatment Condition

Figure 3.8: ConA induces IFN $\gamma$ dependent migration, which can be reduced with IFN $\gamma$ neutralising antibody (a). In splenocyte cultures initially stimulated with ConA for $24 \mathrm{~h}$, then an antiIFN $\gamma$ antibody for $24 \mathrm{~h}$, there was a reduction in the number of migrating CD45+ splenocytes across an epithelial monolayer in vitro (ns). Shown are technical replicates from one experiment $(n=3)$. The data was tested for significance using an ordinary two-way ANOVA with $p=0.055$ I (b) An ELISA of supernatant from the samples in a-c reveals that ConA induces a strong IFN $\gamma$ response in CD45+ cells, and the $\alpha \mathrm{IFN} \gamma$ is effective in neutralising IFN $\gamma$ in these cultures. 


\subsection{Discussion}

The aim of this chapter was to develop an in vitro working model of the choroid plexus. Because of the ChP's anatomical location, it is difficult to study in vivo and requires a large volume of animals. Generally, $\mathrm{ChP}$ research relies on histology to study, which is low throughput, so our goal was to create an isolated system that provides a window into the ChP and allows for higher throughput research. The research focuses on adapting the model described in Monnot and Zheng's., (2013) paper, in which they utilised primary rat choroid plexus epithelial cells and the Z310 cell line to establish Transwell choroidal epithelial cultures for the use in blood-CSF barrier research. As of now, very few other research labs; Monnot and Zheng., (2013), Strazielle et al., (2016) and Vogel et al., (2015) have published working Transwell models of the ChP for the study of leukocyte trafficking, but there is little literature that supports a mouse model, (Lazarevic et al., (2016)) the primary research animal for MS; hence there is absolute need for further development in this field.

Indeed, the choroid plexus is an intrinsic site of leukocyte trafficking into the CNS, acting as an educational gate for immune surveillance under normal physiological conditions, as opposed to the blood-brain barrier which poses as an absolute barrier at homeostasis. ${ }^{37,149,168}$ For an extended period of time, the choroid plexus has been ignored as an important immunological interface despite its daily role in immune functioning; up to 500,000 cells traffic through the ventricles each day. ${ }^{168}$ The key outcome of this chapter was to assess the capacity of this transwell model to mimic homeostatic and inflammatory immune cell trafficking through the choroid plexus, to provide a platform for the study of the new antimigratory drug 'HSI6-35'.

The Transwell cultures relied on three key components to produce an accurate model of the blood-CSF barrier; (I) the layer of fenestrated endothelial cells that line the cerebral vascular walls, (2) the underlying $\mathrm{ChP}$ stromal matrix, in which migrating leukocytes may interact with a small collection of resident immune cells and, (3) the specialised epithelial layer, which forms a tight barrier and governs which cells enter the ventricles. The epithelium prevents free diffusion of blood molecules leaking from the fenestrated endothelium from entering the CSF, but also controls the transport of metabolites and migrating lymphocytes into and out of the CNS. Little is known of the cellular and molecular mechanisms that govern immune cell trafficking across this structure. However, it is suggested that the ChP plays a greater role in homeostatic migration and immunosurveillance than during neuroinflammation. Figure 3.9 describes how this was set up in our model. 
This research began with confirming that CCL5 was non-toxic to murine leukocytes in culture. Previous research by this group has shown that downregulation of CCL5 in EAE mice reduced the number of infiltrating lymphocytes into the CNS, consequently reducing the onset of the disease and its severity. ${ }^{152}$ Because of this work, CCL5 is heavily implicated as one of the primary cytokines mediating cellular migration into the CNS. Although Stanford et al., (2003) describe that CCR5 ligands induce much more potent migratory response from leukocytes in vivo versus in vitro, in keeping with this group's research goal, and the current literature that describes CCL5 as heavily upregulated in the CSF of MS patients, we chose this as our primary chemokine to mediate migration. ${ }^{153,169}$ This research proved that CCL5 was not cytotoxic to splenocytes isolated from healthy C57/BL6] mice up to concentrations of $400 \mathrm{ng} / \mathrm{mL}$, even increasing viability at lower concentrations.

in vivo Choroid Plexus

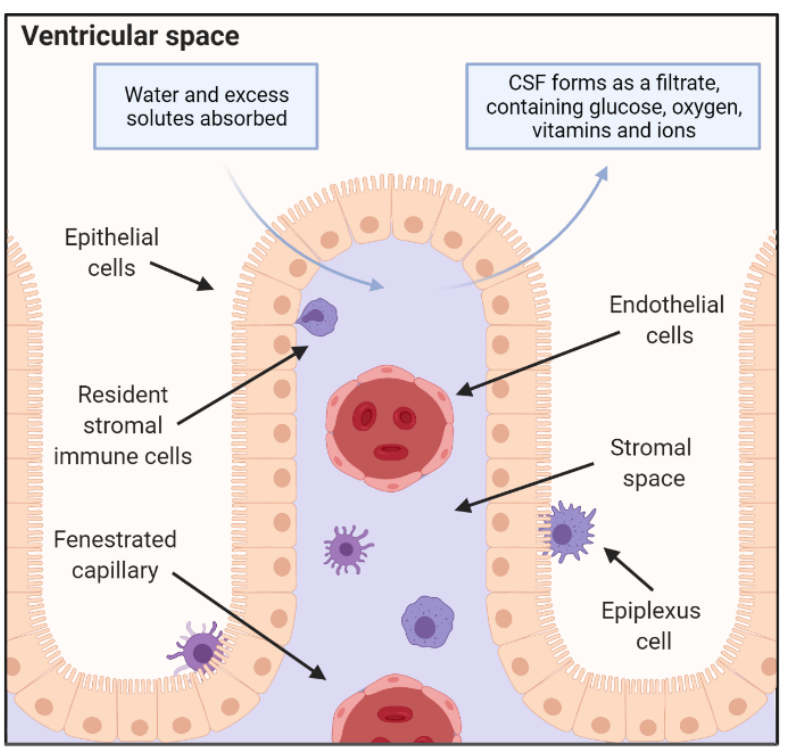

in vitro Choroid Plexus

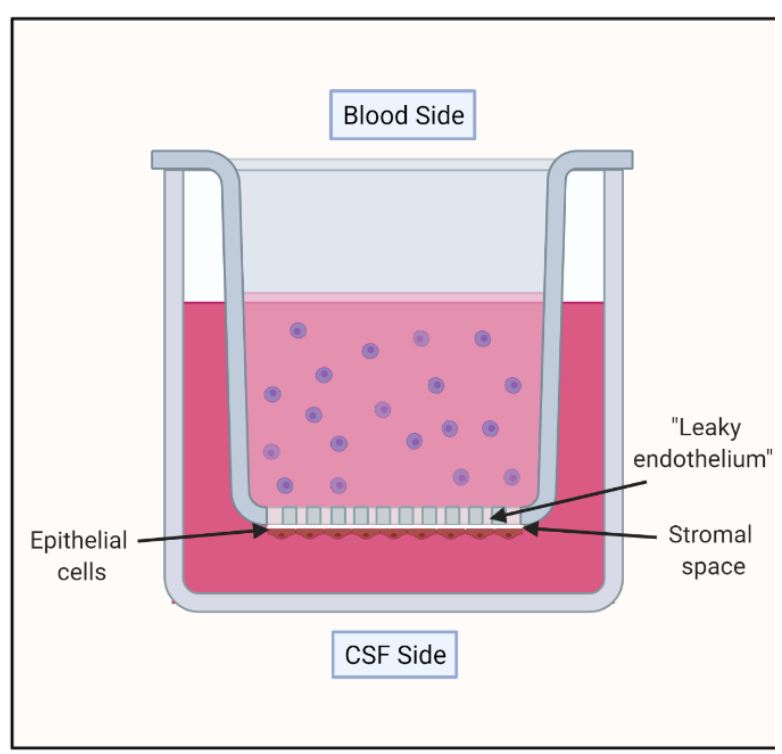

Figure 3.9: Schematic demonstrating how core components of the BCSFB were adapted to an in vitro model. We incorporated the 3 most prominent features of the ChP- the fenestrated endothelium, the stromal matrix, and the tight barrier of epithelial cells.

After selecting an appropriate mediator of chemotaxis for leukocytes, the next step was choosing an appropriate stromal matrix that facilitated an environment for immune cell interactions but also provided key directional cues for the polarised epithelial layer to be cultured upon. Matrigel is a common basement membrane component that has been used to study the invasive and metastatic capacity of cancer cells to disseminate to other sites. ${ }^{170}$ Cells that adopt an aggressive metastatic phenotype can easily navigate the dense molecular network that forms the basement membrane (which normally hinders the movement of cells and other molecules). Although, during inflammation it is not uncommon for leukocytes to release 
matrix degrading enzymes that make the basement membrane more permeable to migrating cells. ${ }^{170}$ Even at its most dilute concentration, leukocytes were unable to permeate the Matrigel coating, and the Matrigel in the media made the samples difficult to safely run through the flow cytometer. Other research groups have incorporated laminin 171,172 or collagen ${ }^{149,173}$ as their stromal matrix mimic. We opted for collagen, which proved to be no issue for all immune subsets to navigate through, but still provided ECPC-4 cells with a components of the basement membrane to grow on. ${ }^{171}$

The epithelial monolayer that formed the tight barrier on our Transwell cultures was one of the most important components of the model. The epithelium prevents free diffusion of blood molecules leaking from the fenestrated endothelium from entering the CSF, but also controls the transport of metabolites and migrating lymphocytes into and out of the CNS. Little is known of the cellular and molecular mechanisms that govern immune cell trafficking across this structure. However, it is suggested that the $\mathrm{ChP}$ plays a greater role in homeostatic migration and immunosurveillance than during neuroinflammation. ECPC-4 cells were chosen in this model as they have been reported to maintain the characteristics of primary choroid plexus epithelium, with the expression of cell-type specific molecules. ${ }^{174}$ In stark contrast, Lazarevic et al., (2016) express concern regarding a comprehensive characterization of this model's expression of TJ markers, $\mathrm{CP}$ epithelial markers, functional barrier characteristics and their capacity to form contact-inhibited monolayers versus primary cell culture. In rebuttal to these points, we performed a number of tests to assess whether these are suitable for this research. Reiterating a benefit of this model that was described earlier, an immortalised cell line reduces the need for large numbers of mice through sourcing primary ChP epithelial cells. This is in keeping with principle of the Three ' $\mathrm{R}$ 's' of animal research. For primary epithelial cultures, 8 to 16 mice are required per preparation, and by troubleshooting this model with over 25 experiments, it becomes hard to ethically justify the number of mice that would be required. 172

The capacity of epithelial cells to form a tight barrier with appropriate junctional complexes is typically assessed via trans-epithelial electrical resistance measurements (TEER). ${ }^{175,176}$ However, this method relies on expensive equipment that our lab did not have access to. We adapted the Evans blue dye assay protocol, which is classically used to study in vivo BBB permeability to measure the permeability and tight barrier function of our model. ${ }^{177}$ Not only did this assay provide key information that our ECPC-4 cells could form an effective barrier and prevent dye penetration into the lower well, we were also able to 
microscopically confirm that ECPC-4 cells seeded onto Transwells did not continue to divide past confluency, indicating the formation of contact inhibited-monolayers.

Once we had confirmed the functionality of our monolayer culture, we next demonstrated that the model was capable of mimicking homeostatic and inflammatory migration of leukocytes across the ChP. To induce an inflammatory response in splenocytes, we stimulated with ConA for 24 hours before putting them into the Transwell. ConA is a T cell activation stimulant, with preferential differentiation capacity toward $\mathrm{T}_{\mathrm{H}} \mathrm{I}$ and $\mathrm{T}_{\mathrm{H}} 17 \mathrm{CD} 4^{+} \mathrm{T}$ cells, so we expected that proliferating $\mathrm{T}$ cells will flood the media with proinflammatory cytokines; primarily IFN $\gamma$, TNF $\alpha$ and IL-6. ${ }^{178}$ In vivo, these responses are often associated with high macrophage and dendritic numbers, which become activated and respond to kill pathogens. Chapter four will investigate the cytokine responses of these cells in our model. Interestingly, between migration experiments, there was significant variability between the number of macrophages and dendritic cells migrating through the monolayer, and if these cells increased in migration in relation to ConA simulation. Because ConA activates $\mathrm{T}$ cells in an antigen independent manner, dendritic cells and macrophages are not activated first and so their activation status directly corresponds to the costimulatory molecules released by $\mathrm{T}$ cells in culture. There may be variation that we have not yet investigated in these responses.

Lider et al., (1990) reported that ConA stimulation induced T cell heparanase expression, which is a key factor of the in vivo system that we wanted to preserve for testing our heparan sulfate mimetic. Because of the substantial research that supports $T$ cell heparanase expression as a mechanism for lymphocyte extravasation into the CNS, we suggest that our ConA-induced increase in migration is at least partially dependent on T cell heparanase expression. This is further explored in later chapters.

ConA-stimulation appeared to sensitize lymphocytes, particularly effector and memory CD4+ and CD8+ $\mathrm{T}$ cells to CCL5 treatment, increasing the number of cells migrating into the lower chamber versus the non-CCL5 counterpart. Studies have shown that in response to ConA stimulation and TCR activation, naïv $T$ cells differentiate into their effector/memory counterparts. As part of sweeping phenotypic changes, they down-regulate receptors for chemokines that are constitutively produced in the tissues, such as CXCR4 and CCR7. ${ }^{179}$ Depending on the extracellular signals and $T_{H} I / T_{H} 2 / T_{H} I 7$ polarization, these lymphocytes acquire chemokine receptors for CCR3, CCR5 or CXCR3, allowing them to migrate to inflamed tissues. ${ }^{179}$ In T cells stimulated with ConA, we hypothesized that the CCR5 receptor is 
upregulated in response, allowing the cells to better respond to the CCL5 gradient in the Transwell culture.

Interestingly, we also demonstrated that ConA induced $\mathrm{CD}^{+}$and $\mathrm{CD}^{+} \mathrm{T}$-cell specific inflammatory migration that was heavily dependent on IFN $Y$ production, which could be partially inhibited with an antiIFNY antibody. These results recapitulate previous findings by this group that reducing the number of IFN $\gamma^{+} \mathrm{CD} 4^{+} \mathrm{T}$ cells may have a positive impact on disease onset and symptom manifestation in EAE mice. ${ }^{180}$ The two-faced role of IFN $\gamma$ in EAE pathology is emphasized by considering initial studies that identified a positive correlation between IFN $\gamma$ an MS/EAE symptomology against contrasting literature that suggests a beneficial role in disease resolution. In healthy mice, immunization with encephalitogenic IFN $\gamma^{+} T_{H} I$ cells was capable of inducing EAE. ${ }^{81}$ Similarly, mice deficient in THI transcription factor 'T-bet' were immune to developing classic EAE. ${ }^{182,183}$ In humans, the pathogenic role of IFN $\gamma$ has been shown in clinical studies that treated MS patients with IFN $\gamma$, severely exacerbating relapses and symptomology. ${ }^{184}$ In contrast, SPMS patients treated with $\alpha \mathrm{IFN} \gamma$ antibodies had showed mild reduction in disease severity. ${ }^{185}$ Other studies have challenged the belief that IFN $N_{Y}$ is purely pathogenic in MS/EAE. For instance, both IFN $N_{Y}-/-$ and IFN $\Gamma R$ -/- mice have been shown to have earlier disease onset, more severe symptoms, and lethality of EAE versus control counterparts. ${ }^{186-190}$ In terms of IFN $\gamma$ therapy, EAE mice treated with IFN $\gamma$ both systemically and intraventricularly have shown positive effects on EAE symptoms. ${ }^{191,192}$ Given the pleiotropic role of IFNY in MS pathology, described by Arellano et al., (20I5), specifically regulating but not completely ablating the IFN $\gamma$ response in the CNS may prove beneficial in dampening the pathogenic CD4 $T_{H} I$ cells that drive major inflammatory events in the CNS. ${ }^{193}$

The benefit of further developing this model in the field of neuroimmunology is clear; the assay has a 'build-a-bear' principle, in which many of the components can be interchanged to study separate aspects of the ChP, even outside the scope of MS. For example, following stroke Martha et al., (2020) report that genes associated with chemokine production are upregulated in the infarct, promoting peripheral immune infiltration. By substituting CCL5 for stroke associated chemokines, CXCL3 and CCL4, this model can simply be adapted to study migration across the ChP in the context of stroke. ${ }^{194}$ Transwell assays classically assess cellular migration through cell counting of the upper and lower chamber, then staining the transwell membrane to count the proportion of cells that are actively migrating. ${ }^{171}$ We have also adapted this assay for flow cytometric analysis which can be further optimised to look at individual subsets of rare cells of interest, or by isolating specific groups of immune cells to study their individual role in disease progression 
in the model. This adaptation provides higher specificity and clarity to the assay than its previous applications have shown. 


\subsection{Summary}

The findings from this chapter support that we were able to reproduce the ChP in an in vitro model. Previously, the $\mathrm{ChP}$ has been hard to research because of its anatomical location, and the requirement for large numbers of animals to assess its functions. Our model was capable of mimicking inflammatory trafficking using ConA stimulation, which was in part due to IFNY released by cells. This trafficking was Tcell specific, similar to the encephalitogenic trafficking in MS and EAE. Upregulated migration in these conditions could be partially abolished with anti-IFNy treatment. 


\subsection{Appendix}

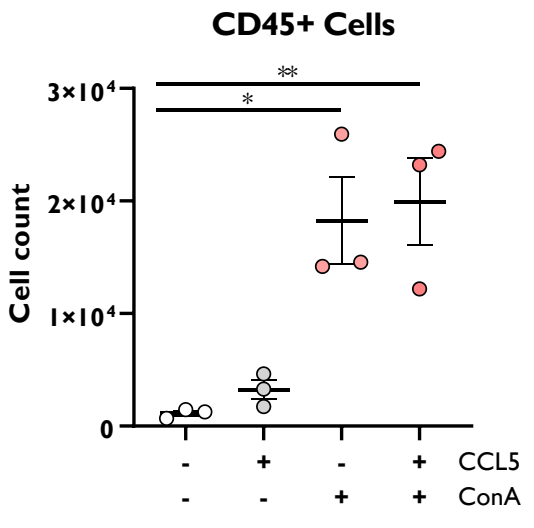

Treatment Condition

B Cells

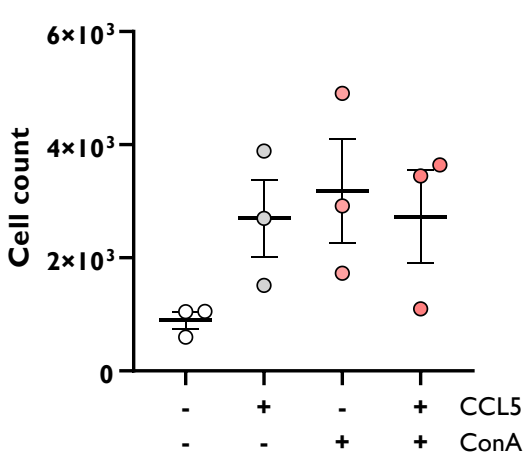

Treatment Condition

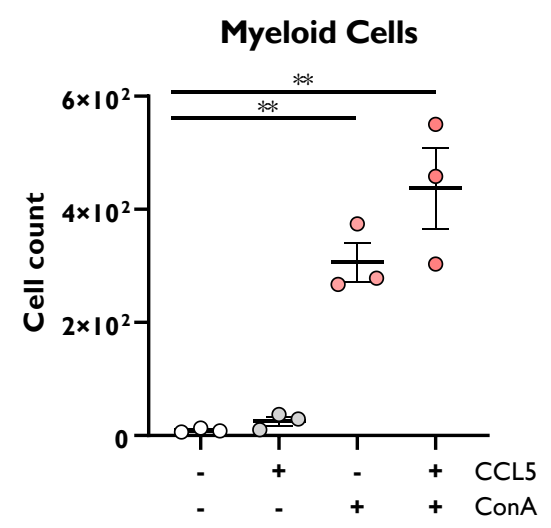

Treatment Condition

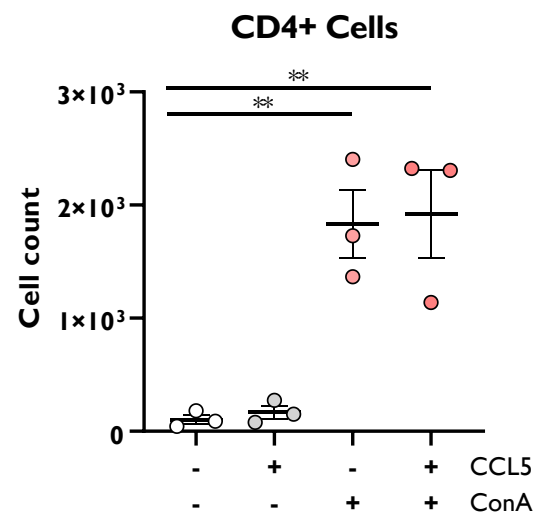

Treatment Condition

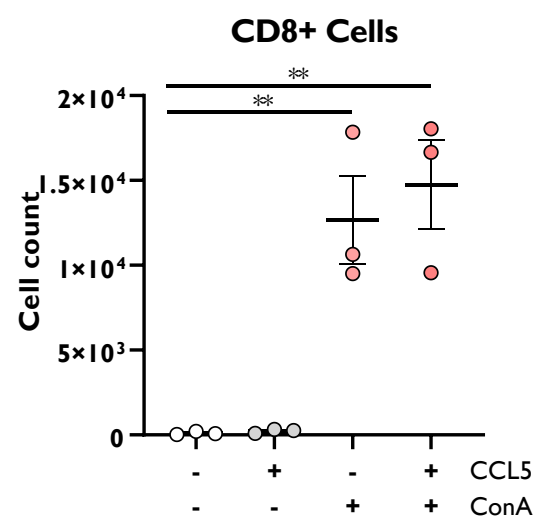

Treatment Condition

Dendritic Cells

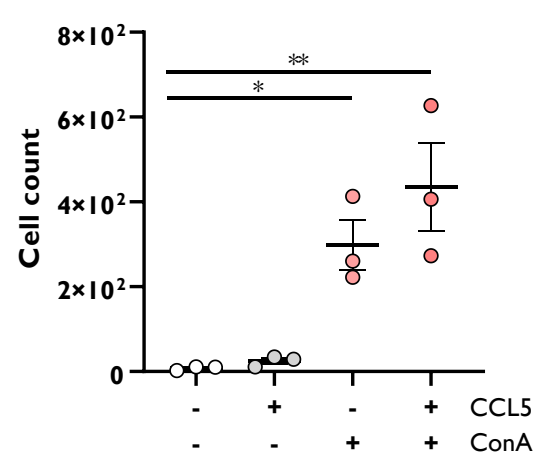

Treatment Condition

Figure 3.10: ConA induces increased migration across our transwell model: Shown are technical replicates from one experiment $(n=3)$. In healthy splenocytes cultured with ConA, there was a significant upregulation in T cell, myeloid cell, and dendritic cell, but not B cell trafficking. A one-way ANOVA was conducted using the cell counts from the bottom well. $p<0.05$. $(*=p<0.05$, ** $=p<0.05, * * *=p<0.01$, **** $=p<$ $0.001)$. 
CD45+ Cells

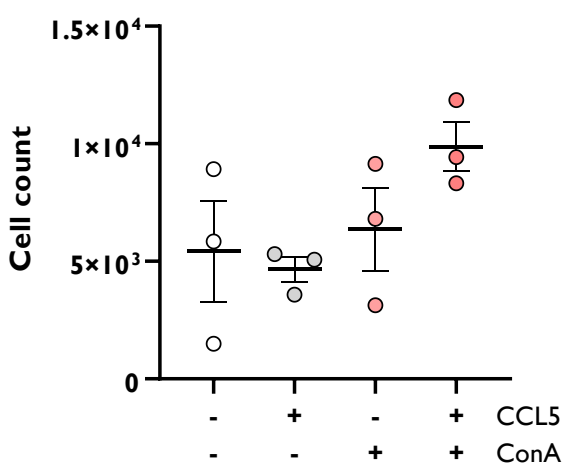

Treatment Condition

B Cells

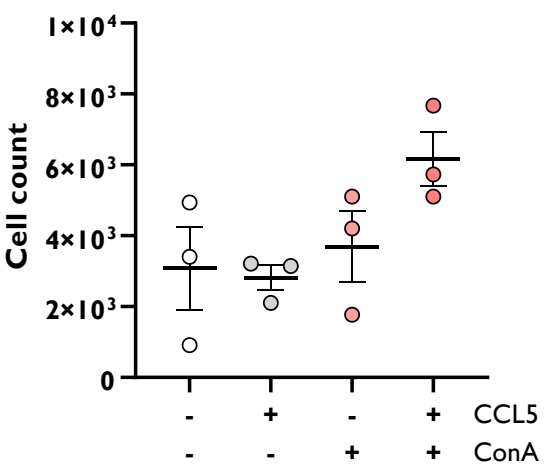

Treatment Condition

Myeloid Cells

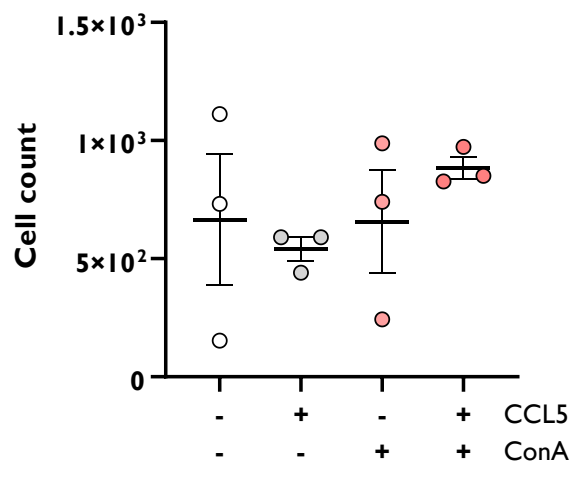

Treatment Condition
CD4+ Cells

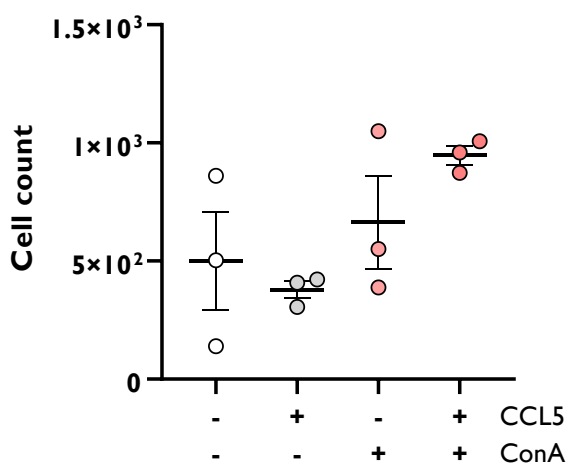

Treatment Condition

CD8+ Cells

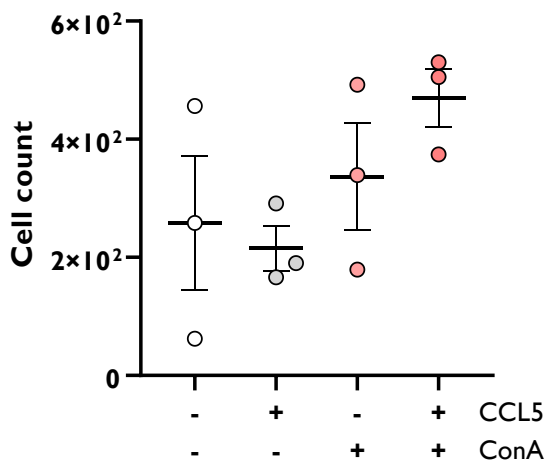

Treatment Condition

Dendritic Cells

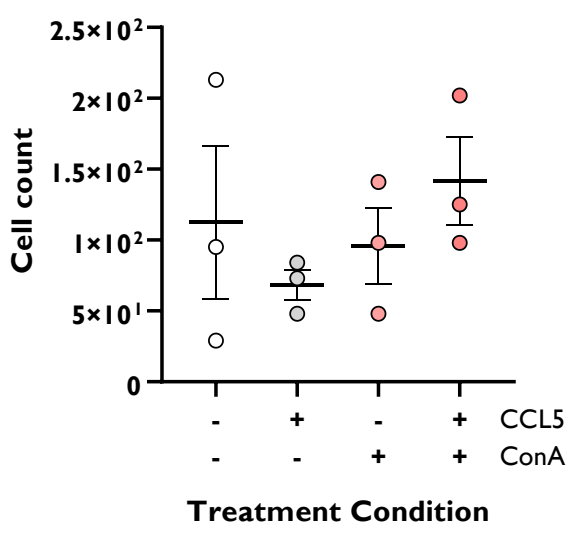

Figure 3.I I: ConA induces increased migration across our transwell model. (a). Shown are technical replicates from one experiment $(n=3)$. In healthy splenocytes cultured with ConA, there is a trend towards increased migration across the monolayer in CD45+ cells versus control. 
Chapter 4: Does the heparan sulfate mimetic 'HS I6 35' affect leukocyte migration in an experimental model of the choroid plexus? 


\section{I. Introduction}

The previous chapter established a functional model of the choroid plexus for the in vitro study of leukocyte trafficking. This chapter will use that in vitro model to investigate the mechanism of action of a novel heparan sulfate mimetic.

HSI6-35 is a heparan sulfate glycomimetic comprised of a dendritic core with four identical sulfated oligosaccharide arms. The commercialization and clinical use of other HS-mimetic competitors currently in development is hindered by the molecular size of these compounds, which have a complex synthesis. ${ }^{145}$ The Zubkova lab at the Ferrier Research Institute developed a library of single entity glycomimetics with simpler synthesis, making the compounds accessible and commercially available. In vitro, they have been shown to employ clustering effects to mimic natural HS molecules in the ECM. ${ }^{195} \mathrm{HSI}$ 6-35 has previously been shown to inhibit heparanase with high potency, with the advantage that it lacks the anti-coagulant activity and cytotoxicity that other HS mimetics have faced. ${ }^{145}$ In vivo, this would likely reduce the side effect profile of HSI6-35 compared to other HS mimetics.

\subsection{Aims}

We propose that HSI6-35 will inhibit migration across this model of the ChP. To test this hypothesis, we will first assess whether the compound is cytotoxic, and select an appropriate dosage for in vitro treatment. Finally, we will evaluate the compound in the transwell system to understand whether it is effective at reducing leukocyte migration across this structure.

\subsection{Specific Aims}

I. To determine whether HSI6-35 affects the viability of isolated splenocytes or the ECPC-4 cellular monolayer at doses that are therapeutic in vivo.

2. To evaluate the cytokines produced by splenocytes and ECPC4 cells treated with HSI6-35.

3. To assess the effect of HSI6-35 on immune cell migration in a transwell model during both homeostatic and inflammatory conditions. 


\subsection{Results}

\subsection{HSI6-35 is non-toxic to murine splenocytes, but exerts mildly cytotoxic effects on ECPC-4 cells}

Previous unpublished work by this group showed that intraperitoneal administration of HSI6-35 at $600 \mu \mathrm{g} /$ mouse daily was effective at reducing disease severity in EAE (unpublished results). To adapt this dosing regimen for in vitro use, we exposed splenocytes and ECPC-4 cells to a wide range of concentrations $(6 \mu \mathrm{g} / \mathrm{mL}, 60 \mu \mathrm{g} / \mathrm{mL}$, and $600 \mu \mathrm{g} / \mathrm{mL})$ for a 24 -hour treatment period to ensure that the compound was not directly cytotoxic to the cells. In this assay, we also treated splenocytes with ConA and IFN $y$ to assess whether these agents altered cell viability in our transwell migration model.

An MTT assay revealed that ECPC-4 cells were significantly impacted by increasing concentrations of HSI6-35, most notably at $600 \mu \mathrm{g} / \mathrm{mL}$, which decreased cell viability to $70.6 \% \pm 3.45$ of the untreated control (Figure 4.la). As in the previous chapter, we performed an adapted Evans blue permeability assay to understand if the extent of cell death was severe enough to interrupt the monolayer after 24 hours of HSI6-35 exposure at $6 \mu \mathrm{g} / \mathrm{mL}, 60 \mu \mathrm{g} / \mathrm{mL}$ and $600 \mu \mathrm{g} / \mathrm{mL}$. Across two experiments, there was no clear increase in dye penetration through the ECPC-4 monolayer into the lower chamber between any of the HSI6-35-treated groups and untreated control (Figure 4.lb). Contrary to the MTT results, it appears that at $600 \mu \mathrm{g} / \mathrm{mL}$ treatment trended towards less dye penetrance versus control. This suggests that the monolayer was becoming more strengthened with HSI6-35 treatment compared to control. Splenocytes showed a much more robust response to HSI6-35 exposure, and no cytotoxicity was detected within the selected dosing range, even when splenocytes were stimulated with ConA or IFNY (Figure 4.2a). Interestingly, EAE splenocytes exhibited a much greater response to ConA stimulation versus healthy splenocytes, consistent with a greater initial inflammatory state of the cells (Figure 4.2b). Based upon these results, we selected $60 \mu \mathrm{g} / \mathrm{mL}(12 \mu \mathrm{M})$ as our optimal concentration in the transwell migration model. 


\section{a. Epithelial Choroid Plexus Cell-4 MTT}

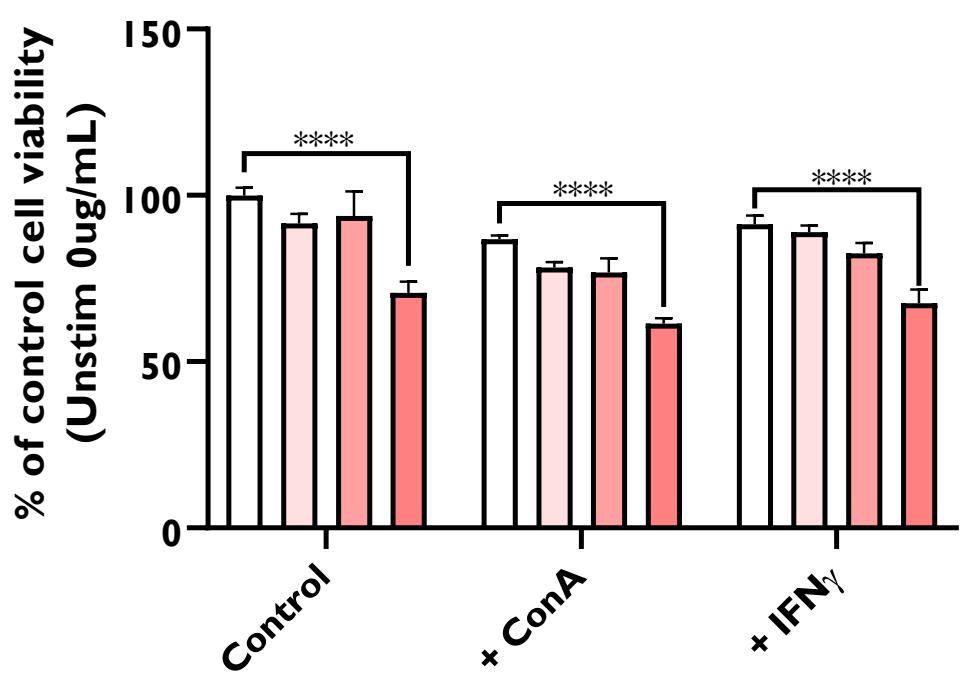

HS I6-35 Concentration

$\square 0 \mu \mathrm{g} / \mathrm{mL}$

$\square 6 \mu \mathrm{g} / \mathrm{mL}$

$60 \mu \mathrm{g} / \mathrm{mL}$

$600 \mu \mathrm{g} / \mathrm{mL}$

\section{Treatment Condition}

\footnotetext{
Figure 4.I: HSI6-35 is mildly cytotoxic to ECPC-4 cells but does not interrupt monolayer permeability. (a) Shown are viability scores as a percentage of untreated control with mean \pm SEM from 2 separate experiments. Results show that increasing concentrations of HSI6-35 for 24h in culture significantly reduced ECPC-4 cell viability from control, regardless of stimulation with ConA or IFN $\gamma$. The data from this experiment was analysed using an ordinary two-way ANOVA with $\alpha=0.05$ $(*=p<0.05, * *=p<0.05$, *** $=p<0.01)$. (b) An Evans blue dye assay was done on the epithelial monolayer after $24 \mathrm{~h}$ treatment to assess whether increasing concentrations of HSI6-35 interrupted the monolayer. There was no significant difference in dye penetration from the untreated control in any of the treatment groups $(n=2)$.
}

b. EBD Assay on ECPC-4 Monolayer

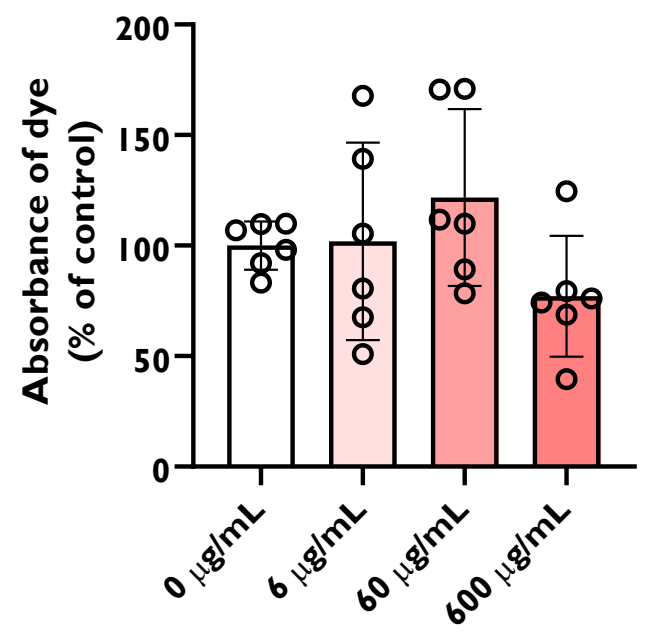

Treatment Condition 
a.

\section{Healthy Splenocyte MTT}

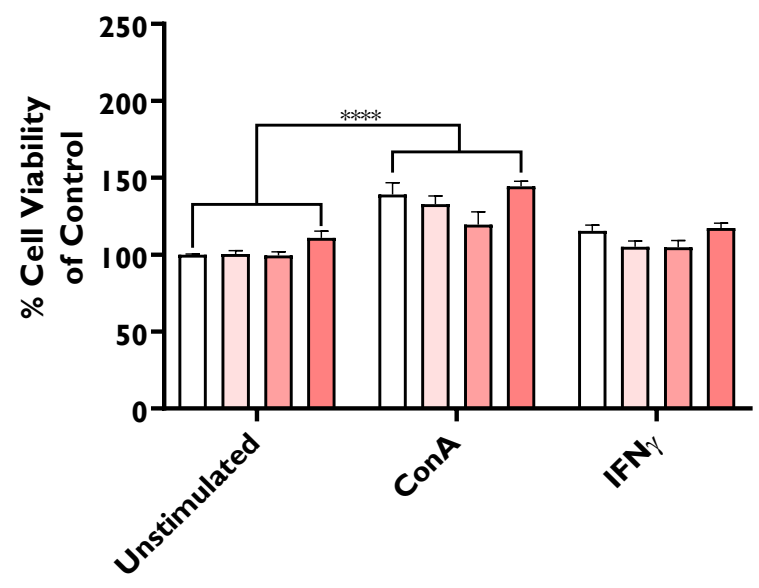

Treatment Condition b. EAE Splenocyte MTT

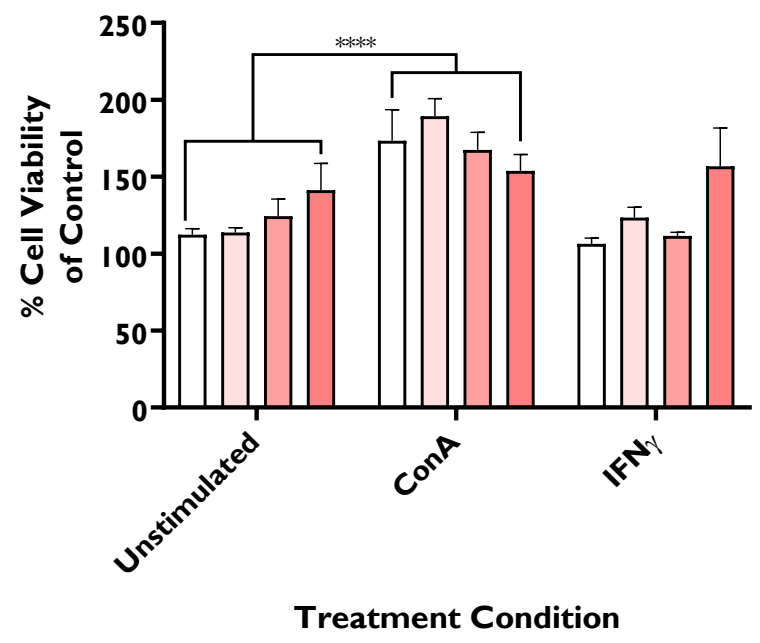

HSI 6-35 Concentration

$\square 0 \mu \mathrm{g} / \mathrm{mL}$
$\square 6 \mu \mathrm{g} / \mathrm{mL}$
$\square 60 \mu \mathrm{g} / \mathrm{mL}$
$\square 600 \mu \mathrm{g} / \mathrm{mL}$

Figure 4.2. HSI6-35 does not exert cytotoxic effects on healthy or encephalitogenic splenocytes. (a) Shown is viability as a percentage of untreated healthy control with mean \pm SEM from 2 experiments $(n=2)$ With the same treatment, HSI6-35 showed no cytotoxicity towards healthy splenocytes after 24 hours. As expected, ConA significantly increased viability from control. (b) When compared to the healthy control, splenocytes isolated from a mouse with active EAE had a greater response to ConA stimulation. However, in all conditions HSI6-35 had no cytotoxic effect on EAE splenocytes. The data from this experiment was analysed using an ordinary two-way ANOVA with $\alpha=$ $0.05(*=p<0.05, * *=p<0.05, * * *=p<0.01)$. 


\subsubsection{HSI6-35 inhibits migration in a Transwell model of the choroid plexus}

As described in Chapter 3, we established a reproducible model of the ChP, adapted slightly from Monnot and Zheng's (2013) paper, and using this model, we evaluated the effect of exposure to HSI6-35 at the concentration identified in 4.3.I on immune cell migration. Murine splenocytes were stimulated with or without ConA for 24 hours and then resuspended in fresh media containing HSI6-35 at $60 \mu \mathrm{g} / \mathrm{mL}$ for 24 hours. In parallel, ECPC-4 monolayers on transwell inserts were treated with HSI6-35 at the same concentration for 24 hours prior to the migration assay. The treated splenocytes were then added to the upper chamber and allowed to migrate for 24 hours in the presence or absence of CCL5 in the lower chamber. The gating strategy for flow cytometric analysis can be found in Figure 4.3. As seen in Figure 4.4a, ConA drastically increased the number of $\mathrm{CD} 45^{+}$cells translocating into the lower chamber. Treatment with HSI6-35 completely ablated this inflammatory trafficking, with the number of cells penetrating the monolayer comparable to the media alone and media + CCL5 conditions. Figure 4.5 are representative flow plots, demonstrating the number of $\mathrm{CD} 45^{+}$cells migrating into the lower chamber when treated with or without HSI6-35. This is an excellent visual representation of the extent that HSI635 inhibits leukocyte migration in this model.

When the specific cellular populations were assessed separately, it was clear that $\mathrm{CD} 4^{+}$and $\mathrm{CD} 8^{+} \mathrm{T}$ cells were the most affected by HSI6-35 especially after ConA stimulation, which induced a dramatic increase in the number of $T$ cells crossing the monolayer (Figure 4.4b). This enhanced migration, which is characteristic of MS, was completely ablated in $\mathrm{CD} 4^{+} \mathrm{T}$ cells and reduced greatly in $\mathrm{CD}^{+} \mathrm{T}$ cells when cells where pre-treated with HSI6-35 in culture (Figure 4.4b). In contrast, ConA stimulation did not enhance the migration of B cells, myeloid cells, and dendritic cells to the lower chamber (Figure 4.4c). However, there was still a notable reduction in DC migration when HSI6-35 was present compared to the control conditions. Taken together, these results strongly suggest that HSI6-35 may be effective in inhibiting migration of autoreactive T lymphocytes into the CNS through the ChP epithelium. 

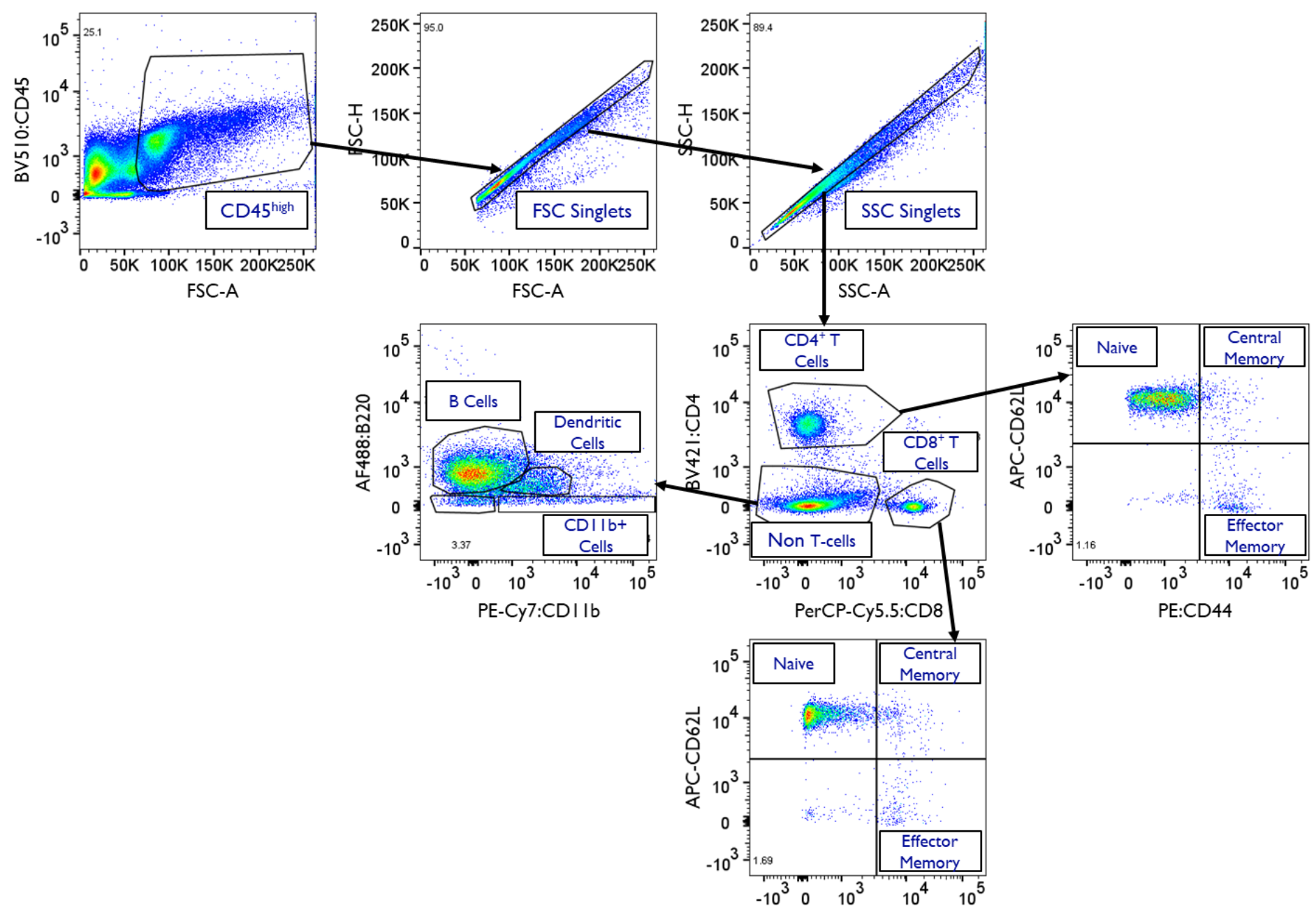

$\mathrm{PE} \cdot \mathrm{CD} 44$

Figure 4.3: Our gating strategy for flow cytometry in chapter 4. All flow cytometry data was assessed using Flowjo v10. Initially, CD45high lymphocytes were gated out, then gated to exclude doublets. All CD45high singlets were placed on a CD4 vs CD8 plot and gated for CD4+ and CD8 ${ }^{+}$ expression. Cells that were non-T cells were then gated based on B220 vs CDI lb expression, with B220high CDI l b- as B cells, B220 int CDI Ib+ as dendritic cells and B220-CDIl $b^{+}$as myeloid cells. $T$ cells were further gated on their expression of CD62L and CD44 with CD62L ${ }^{+} C D 44-$ as naive $T$ cells, $\mathrm{CD} 44^{+} \mathrm{CD} 62 \mathrm{~L}^{+}$as effector/effector memory $\mathrm{T}$ cells and $\mathrm{CD} 62 \mathrm{~L}-\mathrm{CD} 44^{+}$as central memory $\mathrm{T}$ cells. 
a. $\mathrm{CD}^{\circ}{ }^{+}$Cells

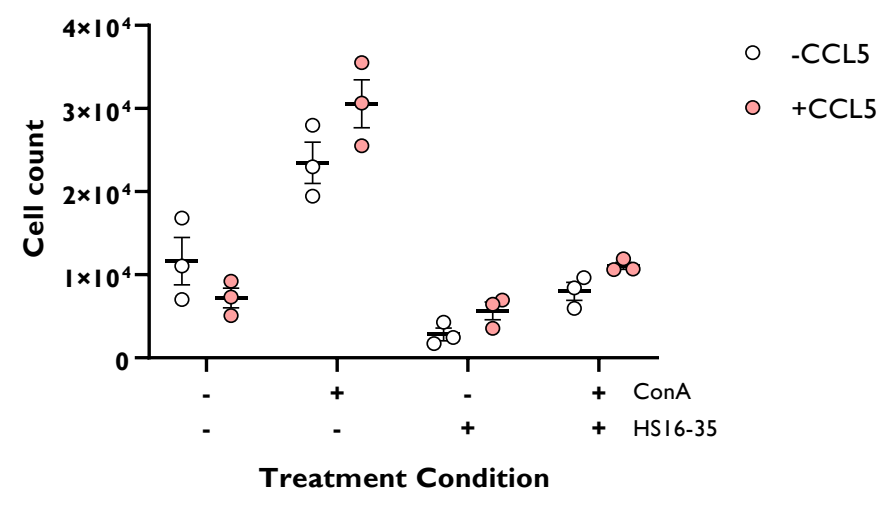

b.

$\mathrm{CD}^{+}{ }^{+}$T Cells

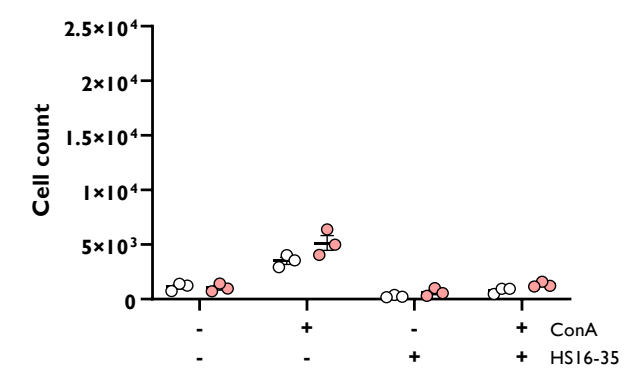

$\mathrm{CD8}^{+} \mathrm{T}$ Cells

- $-\mathrm{CCL} 5$

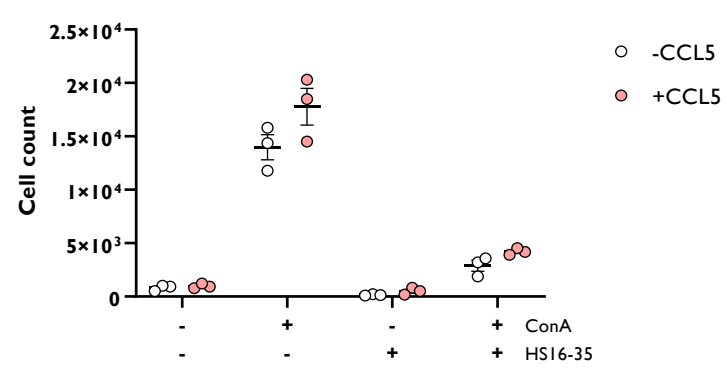

c.

B220 ${ }^{+}$Cells

Myeloid Cells
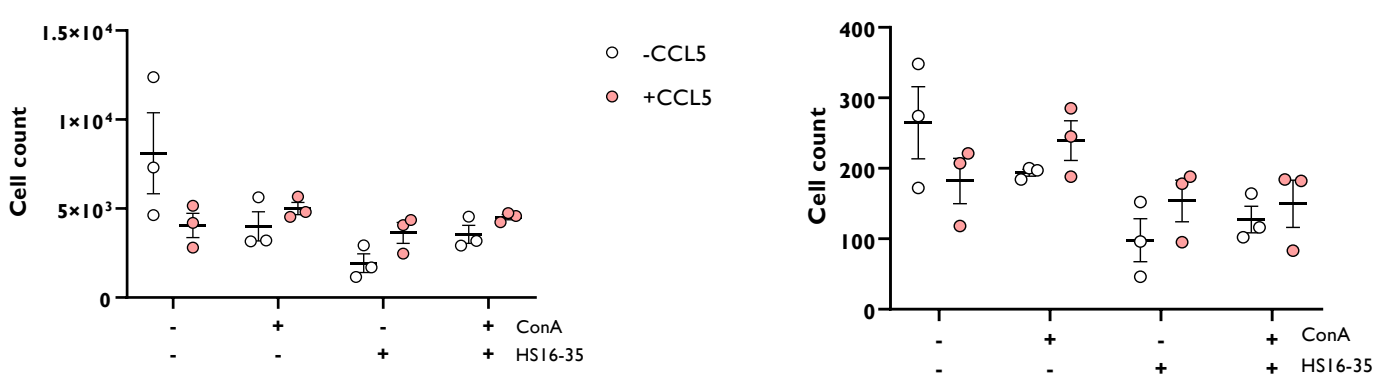

- - CCL5

- +CCL5

Dendritic Cells

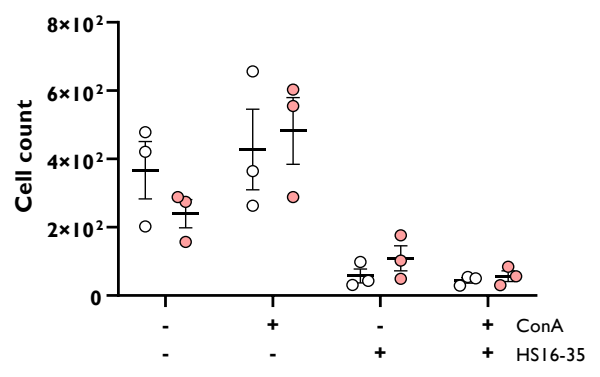

Figure 4.4: HSI6-35 inhibits ConA-mediated migration of CD45+ leukocytes across an

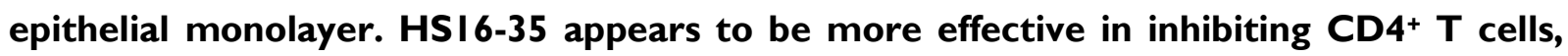
CD8 $^{+} \mathbf{T}$ cells and DC (a) Shown are individual data points from one experiment \pm SEM. When splenocytes are treated with ConA, there is a significant increase in migration across the epithelial monolayer. This inflammatory trafficking is completely ablated in cultures pre-treated with HSI6-35, which returns migration to control levels. The data from this experiment was analysed using an ordinary two-way ANOVA with $\alpha=0.05(*=p<0.05, * *=p<0.05, * * *=p<0.01)$. (b) CD4 $^{+}$and CD8 $^{+} \mathrm{T}^{*}$ 
cells show strong upregulation in trafficking in response to ConA stimulation, but this could be completely inhibited with HSI6-35 treatment. These results were analysed using an ordinary two-way ANOVA with $\alpha=0.05(*=p<0.05, * *=p<0.05$, *** $=p<0.01)$. (c) B220+, myeloid cells and dendritic cells show no response to ConA stimulation, but still respond to HSI6-35 treatment by reduced trafficking $(\mathrm{n} / \mathrm{s})$.

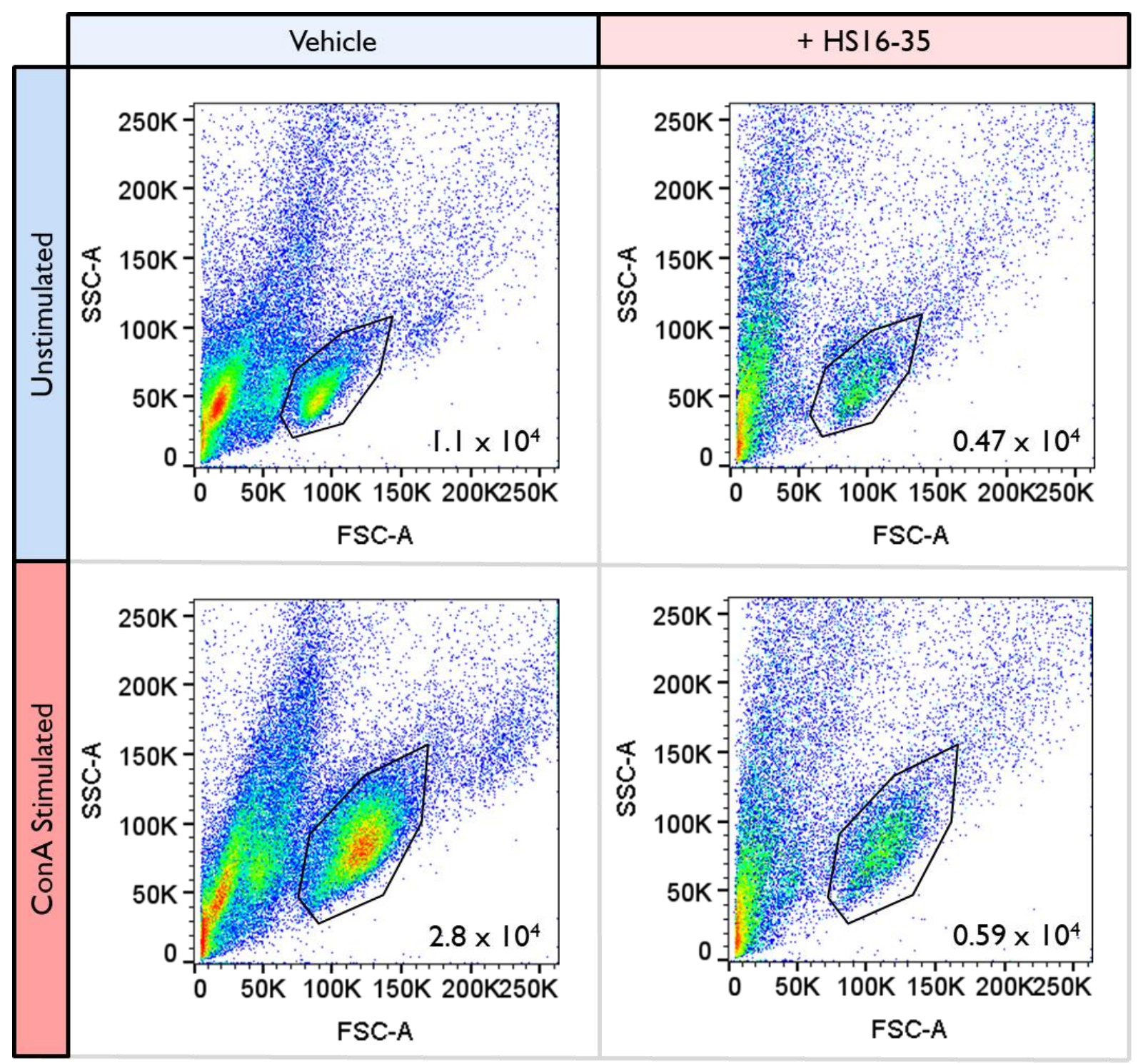

Figure 4.5: ConA induces an increase in migration across a Transwell culture which is mitigated by HSI6-35. Shown here are four representative flow plots from a migration experiment in which the effects of $48 \mathrm{~h} \mathrm{HSI6-35}$ pre-treatment on migration into the lower well was examined. The black line shows the lymphocyte population, and the number in the corner indicates the number of cells in that population. What can be observed from these flow plots is that ConA stimulation increases the raw number of cells migrating through the Transwell. Pre-treatment with HSI6-35 shows a visual reduction in migration through the Transwell in both Unstimulated and ConA stimulated conditions. 
The flow cytometry panel included CD44 and CD62L to allow a better understanding of the activation states of the $\mathrm{CD}^{+}$and $\mathrm{CD} 8^{+} \mathrm{T}$ cells migrating through the epithelial monolayer (Figure 4.6a, 4.6b). In mice, CD4 and CD8 T cells can be categorized into naïve, effector, or memory phenotypes based on their differential expression of CD44 and CD62L. CD44-CD62 $\mathrm{L}^{+}$cells are considered naïv $\left(\mathrm{T}_{\mathrm{N}}\right)$, with a shift to $\mathrm{CD} 44^{+} \mathrm{CD} 62 \mathrm{~L}-$ considered effector or effector memory $\left(\mathrm{T}_{\mathrm{E}} / \mathrm{EM}\right)$. Finally, $\mathrm{CD} 44^{+} \mathrm{CD} 62 \mathrm{~L}^{+}$cells are considered central memory $\left(\mathrm{T}_{\mathrm{CM}}\right) .{ }^{196}$ The $\mathrm{ChP}$ stroma houses a population of resident $\mathrm{CD} 4^{+}$effector memory $\mathrm{T}$ cells, whereas $\mathrm{CD} 4^{+} \mathrm{T}$ cells in the CSF generally express central memory phenotypes. ${ }^{39} \mathrm{We}$ expected to see these populations represented more heavily than naïve populations.

\subsubsection{1. $\quad \mathrm{CD}^{+} \mathrm{T}$ cells}

Using these markers, we found that naïve $C D 4^{+} T$ cell $\left(C D 62 L^{+} C D 44\right)$ migration was not enhanced by ConA stimulation, which is not surprising given that ConA induces $\mathrm{T}$ cell activation. ConA increased $\mathrm{CD}_{4}{ }^{+}\left(\mathrm{T}_{E}\right.$ or $\mathrm{T}_{C M}$ ) but not naïve populations (Figure 4.6a). The average number of total (upper + lower chamber) $\mathrm{CD}^{+}$naïve $\mathrm{T}$ cells in the control, vehicle-treated condition was $5.4 \times 10^{3} \pm 672$, whereas with ConA stimulation, this number decreased to $4.3 \times 10^{3} \pm 640$. With HSI6-35 treatment, the total number of naïve CD4+ $\mathrm{T}$ cells sits at $6.8 \times 10^{3} \pm 492$ and does not decrease with ConA treatment maintaining an average of $7.0 \times 10^{3} \pm 538$ (Figure 4.7a). In addition, naive $\mathrm{CD}^{+} \mathrm{T}$ cells did not respond to CCL5 stimulation as there was no change in migration across the ECPC-4 monolayer between \pm CCL5 stimulated conditions (Figure 4.6a).

In contrast to naïve $\mathrm{CD}^{+}{ }^{+} \mathrm{T}$ cells, $\mathrm{CD}^{+} \mathrm{T}_{\mathrm{E}}$ cells displayed increased trafficking across the ECPC-4 monolayer in response to ConA stimulation. In the media-alone and media + CCL5-stimulated conditions, there was no difference in migration, however when stimulated with ConA there was an observable increase in the number of $C D 4^{+} T_{E}$ cells penetrating the monolayer in response to CCL5 stimulation (Figure 4.6a). HSI6-35-treated $\mathrm{CD}^{+} \mathrm{T}_{\mathrm{E}}$ cells showed low-level trafficking, comparable to the media alone condition, and did not show increased trafficking in response to CCL5 stimulation (Figure 4.6a). Interestingly, HSI6-35 treatment of previously Con-A stimulated cultures clearly decreased the number of migrating $\mathrm{CD}^{+} \mathrm{T}_{\mathrm{E}}$ cells from the ConA-alone stimulated condition, with these cells no longer showing a sensitized response to CCL5 stimulation (Figure 4.6a). Figure 4.7a shows the total number of CD4+ $T_{E}$ cells in the transwell culture (upper + lower chambers). ConA stimulation clearly increased the number of $\mathrm{CD}^{+} \mathrm{T}_{\mathrm{E}}$, but this effect was amplified when cells were also stimulated with CCL5, which further 
increased the number of effector cells (Figure 4.7a). This CCL5-mediated response was not seen in cultures treated with ConA and HSI6-36.

Finally, central memory $\mathrm{CD} 4^{+} \mathrm{T}$ cells show the most dramatic increase in migration across the monolayer in response to ConA stimulation. Similarly, to the trend seen with $\mathrm{T}_{\mathrm{EM}} \mathrm{CD} 4^{+}$cells, media $+\mathrm{CCL5}$ stimulation was not sufficient to increase migration of $\mathrm{CD}^{+}{ }^{+} \mathrm{TCM}$ into the lower chamber compared to media alone (Figure 4.6a). When previously stimulated with ConA, $C D 4^{+} T_{C M}$ showed a sensitized, greater migratory response to CCL5 stimulation versus ConA alone. When stimulated with HSI6-35 alone, these cells showed a low migratory response, comparable to the media alone and media + CCL5. Interestingly, HSI6-35 treatment of ConA-stimulated cultures was also capable of reducing CD4+ $\mathrm{T}_{\mathrm{CM}}$ migration to levels comparable to the media alone control; however, these cells still demonstrated the CCL5 sensitized-migration characteristic of ConA-stimulated migration (Figure 4.6a). In regard to the total number (upper + lower chambers) of $\mathrm{CD}^{+} \mathrm{T}_{\mathrm{CM}}$ in each transwell culture, in wells not stimulated with ConA, the $C D 4^{+} T_{C M}$ population was virtually absent compared to ConA-stimulated wells (Figure 4.7a). In addition, in ConA-stimulated wells, $C D 4^{+} \mathrm{T}_{C M}$ show increased cell counts in response to CCL5 similarly to CD4+ $T_{E}$ cells (Figure 4.7a). There appears to be no difference in the overall cell count between ConA stimulated \pm HSI6-35 conditions, however the CCL5-dependent increase in CD4+ $\mathrm{T}_{\mathrm{CM}}$ cell number is not present in HSI6-35 treated wells (Figure 4.7a).

\subsubsection{2. $\quad \mathrm{CD8}^{+} \mathrm{T}$ cells}

With the same markers, we found that in contrast to $C D 4^{+} T_{N}$ cells, naive $C D 8^{+} T$ cells greatly upregulated trafficking across the transwell culture in response to ConA stimulation (Figure 4.6b). This finding was unexpected, because generally naïve cells are not capable of entering the CNS. Unlike other T cell subsets, the number of $C D 8^{+} T_{N}$ cells trafficking into the lower chamber was not altered with CCL5 stimulation, regardless of stimulation with ConA or treatment with HSI6-35. When treated with HSI6-35 alone, $\mathrm{CD}^{+} \mathrm{T}_{\mathrm{N}}$ trafficking decreased slightly from the media-alone controls (Figure 4.6b). When previously stimulated with ConA, then treated with $\mathrm{HSI6-35,} \mathrm{CD}^{+} \mathrm{T}_{\mathrm{N}}$ cells decreased dramatically in migration compared to ConA-stimulated, untreated wells (Figure 4.6b). Unexpectedly, the total number of CD8 ${ }^{+}$ $T_{N}$ cells increased in response to ConA stimulation, and this increase is not altered by HSI6-35 treatment (Figure 4.7b).

Both CD8 ${ }^{+} T_{E}$ and $T_{C M}$ showed a similar pattern in this model. With media alone or media $+C C L 5$, there was low level migration across the monolayer, which was not specific to CCL5 stimulation (Figure 4.6b). 
With ConA stimulation, both groups expanded rapidly in total cell number, and the number of cells that migrated through the monolayer (Figure 4.6b, Figure 4.7b). The proliferation seen in ConA stimulated wells increased with CCL5 stimulation, similar to the $C D 4^{+} T_{E}$ and $T_{C M}$ subgroups (Figure 4.7b). Both $C D 8^{+} T_{E}$ and $T_{C M}$ subgroups became sensitized to CCL5 stimulation when costimulated with ConA, increasing migration across the transwell culture from ConA-stimulated wells alone (Figure 4.6b). Again, treatment with HSI6-35 caused complete ablation of Con-A-mediated trafficking in these subsets, returning migration across the monolayer to a level comparable to the media alone control (Figure 4.6b). Interestingly, HSI6-35 treatment also reduced the total number of $T_{E}$ and $T_{C M}$ in culture when previously stimulated with ConA. HSI6-35 treated samples also lack the CCL5-dependent increase in cell number seen when stimulated with ConA alone (Figure 4.7b). 

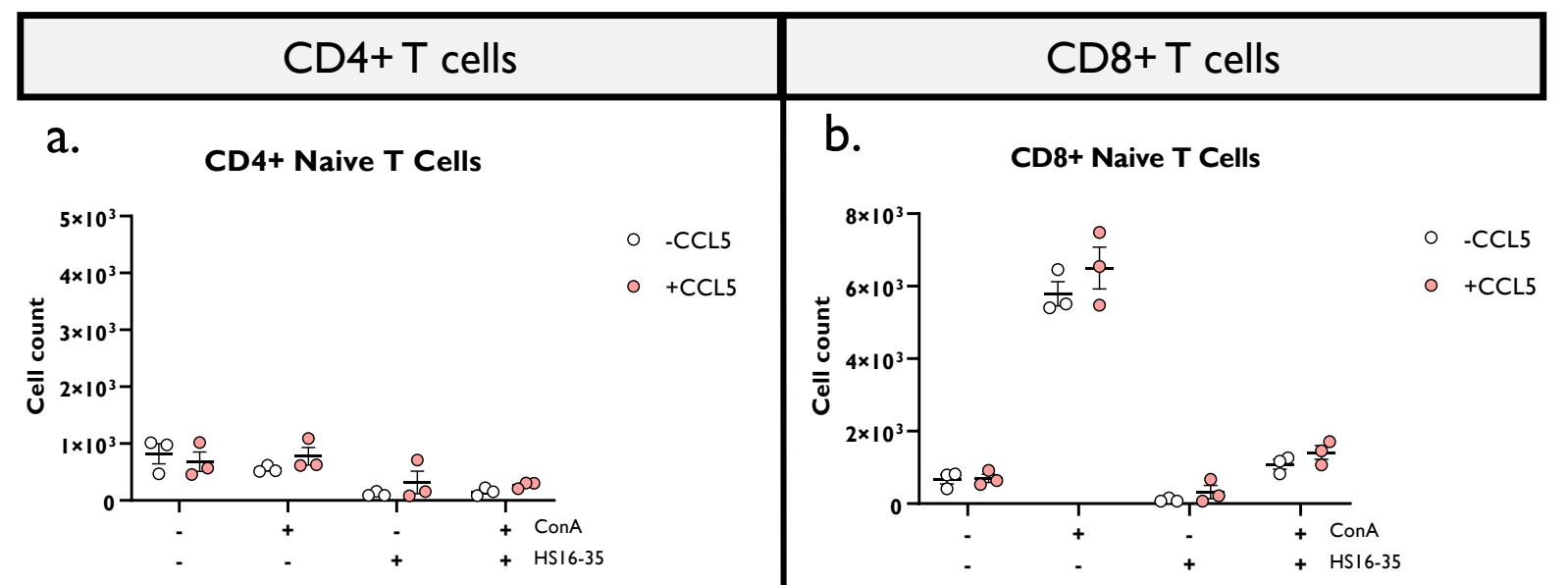

b. CD8+ Naive T Cells

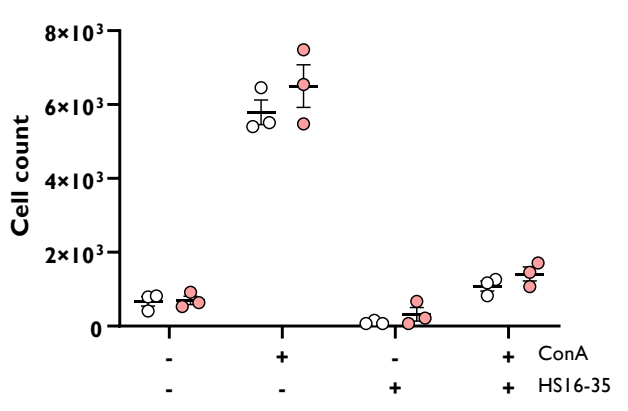

CD4+ Effector Memory T Cells

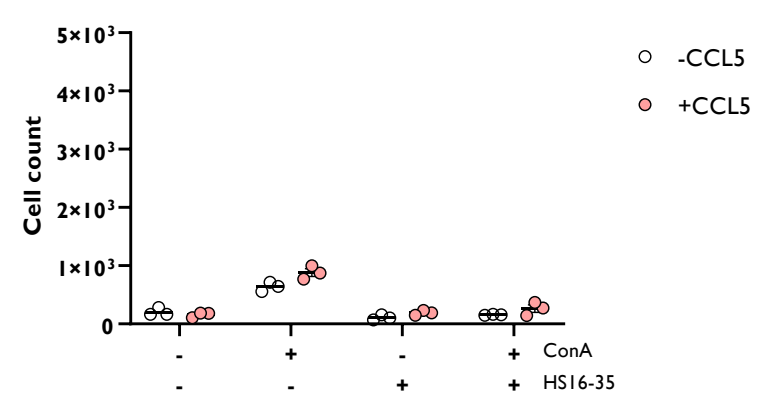

CD8+ Effector Memory T Cells

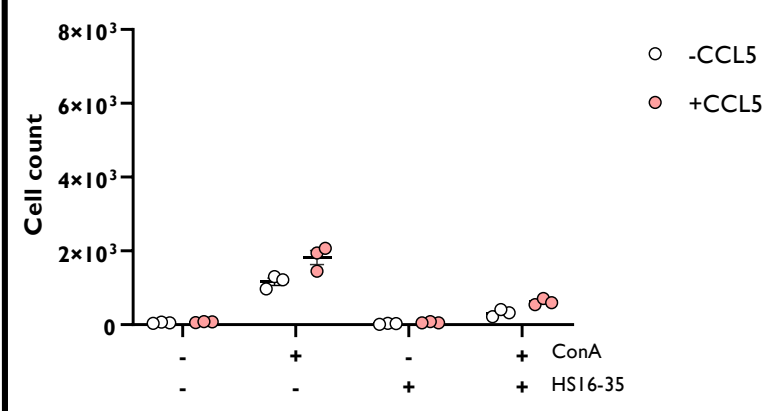

CD4+ Central Memory T Cells

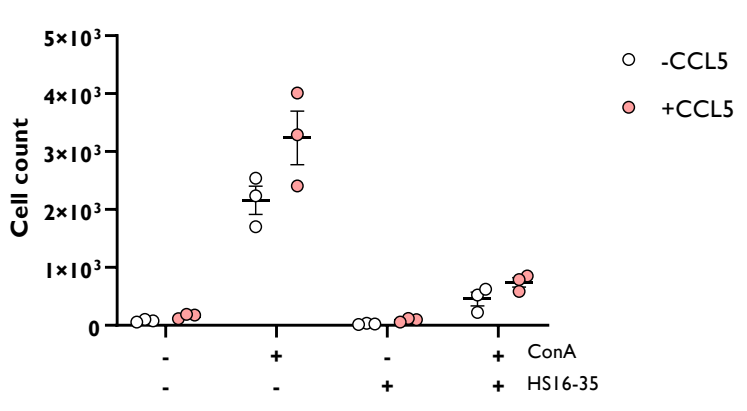

CD8+ Central Memory T Cells

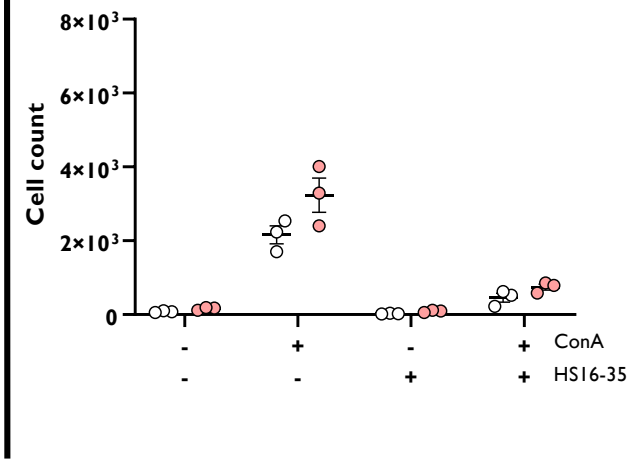

Figure 4.6: HSI6-35 inhibits migration of on CD4+ $T_{E M}, T_{C M}$ and $C D 8^{+} T_{N}, T_{E M}, T_{C M}$ but not CD4 ${ }^{+} \mathbf{T}_{\mathbf{N}}$ subsets. (a) Shown are individual data points from one experiment \pm SEM. When splenocytes are treated with ConA, there is an increase in central memory and effector/effector memory but not naive $\mathrm{CD}^{+} \mathrm{T}$ cell migration across the epithelial monolayer. This inflammatory trafficking is completely inhibited in cultures pre-treated with HSI6-35, which returns migration to the level of media alone conditions. (b) Shown are individual data points from one experiment \pm SEM. When splenocytes are treated with ConA, there is an increase in central memory, effector/effector memory and naïv $\mathrm{CD} 8^{+} \mathrm{T}$ cell migration across the epithelial monolayer. This inflammatory trafficking is completely inhibited in cultures pre-treated with HSI6-35, which returns migration to the level of media alone conditions. 

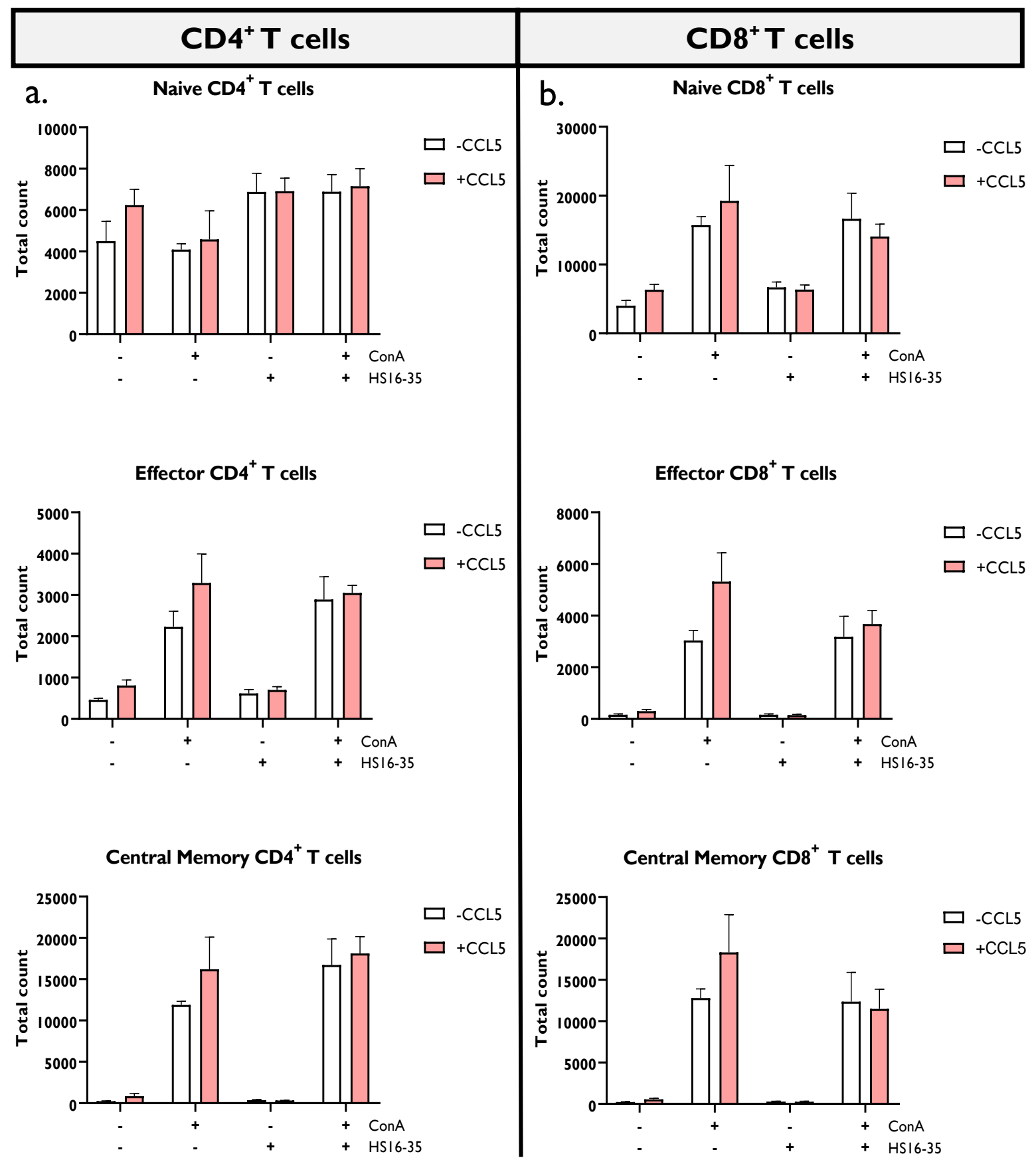

Figure 4.7: ConA increases the total number of effector and central memory CD4 ${ }^{+} \mathrm{T}$ cells but decreases the total number of effector and central memory CD8 ${ }^{+} T$ cells . (a) Shown are the total number (upper + lower wells) of $\mathrm{CD}^{+}$cells in the transwell culture following stimulation \pm ConA and treatment \pm HSI6-35. Each bar represents the mean of one experiment with triplicate values \pm SEM. Naïve CD4+ $T$ cells appear to increase in number following treatment with HSI6-35, regardless of treatment with or without ConA. Both effector CD4+ and central memory CD4+ T cells are increased in number following treatment with ConA. Central memory CD4+ T cells appear to be mildly upregulated in response to HSI6-35 treatment. (b) Shown are the total number of CD8+ $\mathrm{T}$ cells in the transwell culture following stimulation \pm ConA and treatment \pm HSI6-35. Each bar represents the mean of one experiment with triplicate values \pm SEM. Interestingly, ConA stimulation increased the total number of Naïve CD8 ${ }^{+} \mathrm{T}$ cells, regardless of stimulation with or without HSI6-35. In contrast, 
whilst ConA increases the number of effector and central memory CD8 ${ }^{+} \mathrm{T}$ cells, this increase is dampened slightly with HSI6-35 treatment. 
Chapter 3 outlined a clear role for IFN $\gamma$ in MS pathogenesis and in our model, so we focused heavily on its involvement in HSI6-35's effects in this chapter. To determine whether IFN $\gamma$ was involved in the ConAinduced immune cell migration in this model, healthy murine splenocytes and the ECPC-4 monolayer were stimulated with IFN $\gamma$ for 24 hours. Following this, the media was aspirated, and the ECPC-4 cells treated with HSI6-35 for a further 24 hours before adding splenocytes to the upper chamber of the transwell. As $\alpha \mathrm{IFN} \gamma$ treatment had previously been shown to reduce migration in this model, we used ConA stimulation and $\alpha \mathrm{IFN} \gamma$ stimulated splenocytes as controls to assess the extent that HSI6-35 reduced migration in this model (3.3.5)

Figure 4.8. shows the cell counts of all CD45+ leukocytes that successfully migrated into the lower chamber. Although the cell-specific effects of each stimulatory condition is dampened here, there is a dramatic decrease in the total number of cells migrating across the monolayer when pre-treated with HSI6-35, despite prior treatment with or without IFN $\gamma$. This decrease in migration is below that of the homeostatic, media alone condition. When each immune cell subset was assessed separately, $\mathrm{CD} 4^{+}$and $\mathrm{CD}^{+} \mathrm{T}$ cells were again identified as most affected by HSI6-35 treatment, especially following prior stimulation with IFN $\gamma$ (Figure 4.9). When $\mathrm{CD}^{+}$and $\mathrm{CD}^{+} \mathrm{T}$ cells were stimulated with IFN $\gamma$, there was mild increase in trafficking across the monolayer, consistent with findings from chapter 3 (Figure 4.9). These cells also appeared more sensitized to CCL5 stimulation than media alone stimulated wells, with increased migration between the -CCL5 and +CCL5 IFNy stimulated wells. T cell migration enhanced by IFN $\gamma$ was not as prominent as ConA-stimulated wells, which showed increased migration from the media alone and IFN -treated wells, especially notable with the $\mathrm{CD}^{+} \mathrm{T}$ cell population (Figure 4.9). This is not surprising, as we have previously demonstrated that ConA-induced T cell specific increase in migration. Here, the $\alpha$ IFN $\gamma$ antibody exerted a mild effect on $\mathrm{CD}^{+} \mathrm{T}$ cell migration but not $\mathrm{CD} 4^{+}$cells (Figure 4.9). HSI6-35 was particularly effective in reducing T cell migration below baseline (media alone) and the $\alpha \mathrm{IFN} \gamma$ control, additionally removing the CCL5-sensitization seen when stimulating the leukocytes with IFNY alone (Figure 4.9).

When examining non-T cell populations, the effect of IFNY on migration was completely absent. Whereas in $\mathrm{B}$ cells, IFN $y$ did not alter migration across the monolayer from the media alone control, in both myeloid cells and dendritic cells, IFN $N_{\gamma}$ stimulation clearly decreased migration (Figure 4.10). As previously described, ConA did not affect the migration of non-T-cell subsets, a result that is consistent in these findings. Finally, although there was no increase in B cell, myeloid cell or dendritic cell migration in 
response to these inflammatory stimuli, when treated with HSI6-35, these subsets were all dramatically decreased in migration across the monolayer from the media alone control (Figure 4.10). 
CD45+ Cells

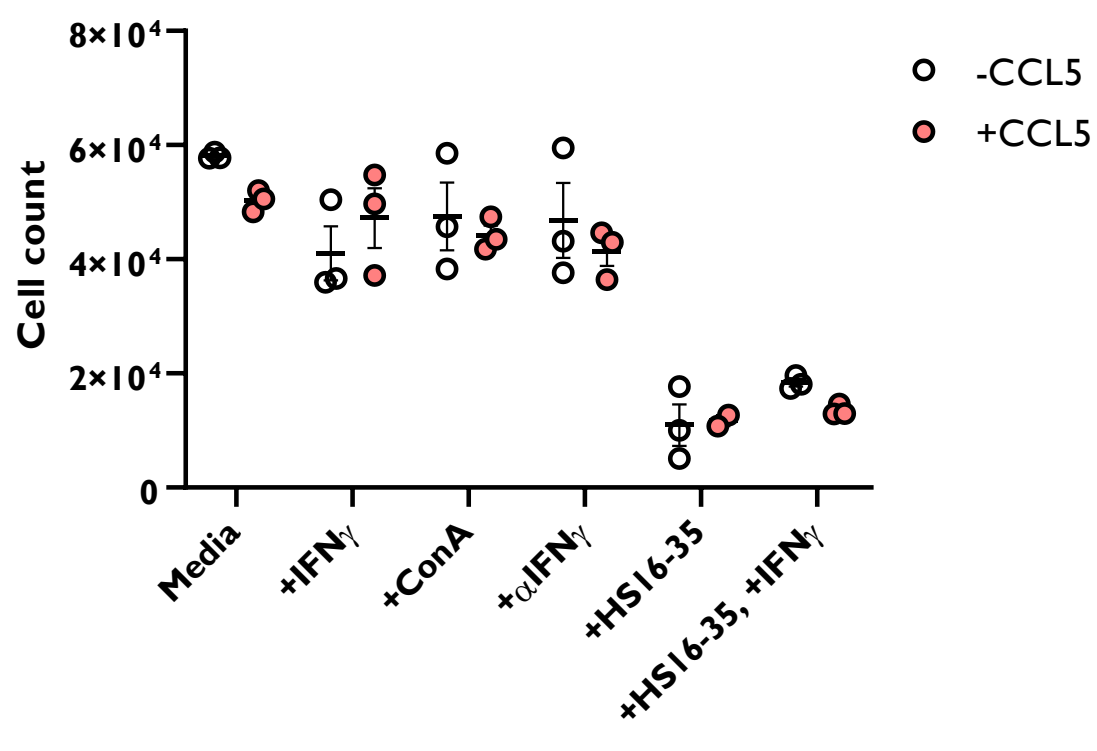

Treatment Condition

Figure 4.8: HSI6-35 reduced general leukocyte migration through a transwell model of the ChP. Shown are experimental values of the number of cells crossing into the lower chamber with the mean \pm SEM. Although IFN $\gamma$, ConA and $\alpha$ IFN $\gamma$ appear not to alter the migration of CD45+ cells across the ECPC-4 monolayer, 24h HSI6-35 treatment at $60 \mathrm{ug} / \mathrm{mL}$ is effective in reducing migration into the lower chamber from the media alone condition. 


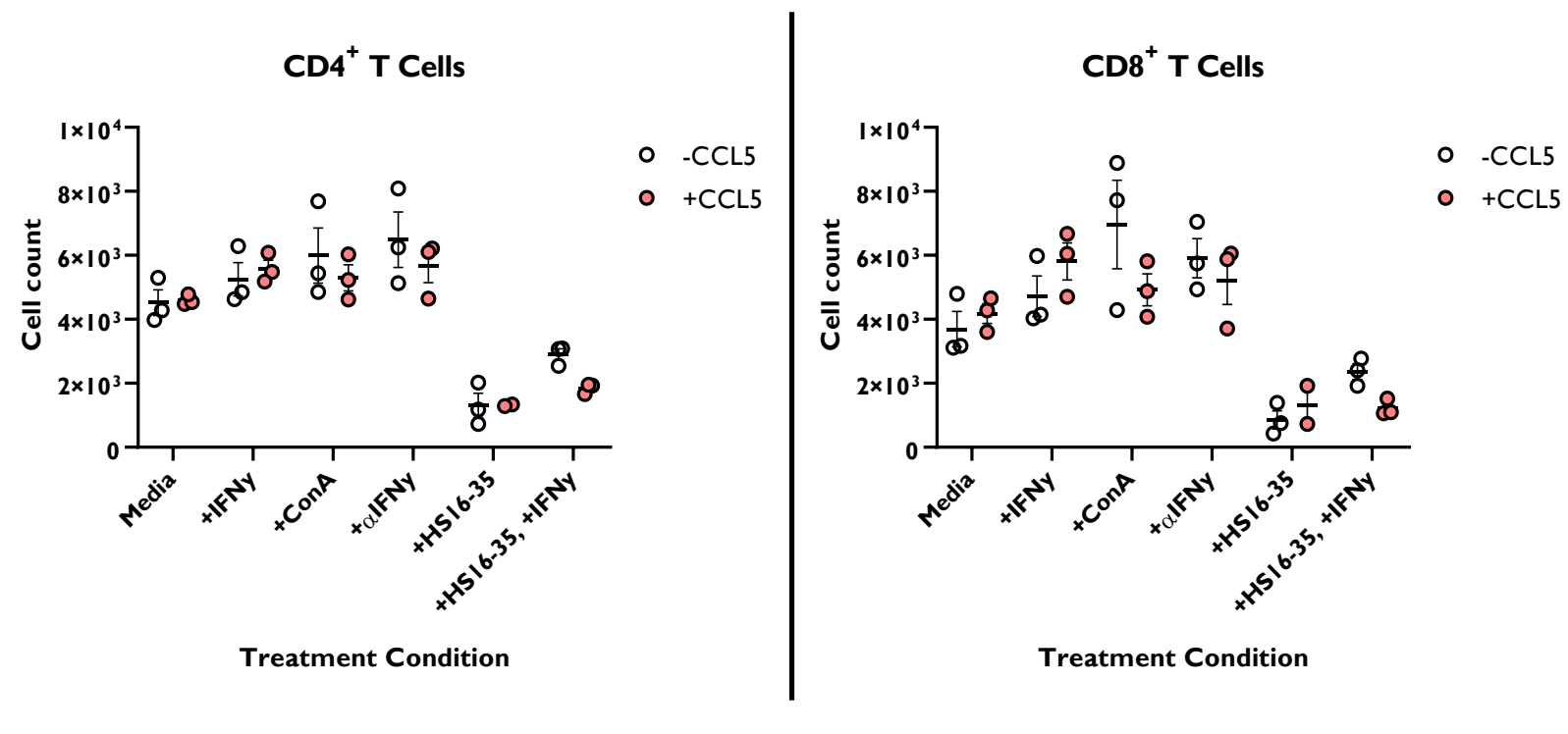

Figure 4.9: HSI 6-35 reduced IFN $\gamma$-mediated CD4+ and CD8+ $\mathrm{T}$ cell migration through a transwell model of the ChP. Shown are experimental values of the number of cells crossing into the lower chamber with the mean \pm SEM. An increase in migration is seen with IFNy stimulation from the media-alone control, and an even greater increase with ConA stimulation. $\alpha \mathrm{IFN} \gamma$ treatment partially reduced $\mathrm{CD}^{+}$but not $\mathrm{CD}^{+}$trafficking into the lower well. When cultures were treated with HSI635 , there was a notable decrease in the number of cells penetrating the monolayer, below the mediaalone control. This HSI6-35 mediated decrease is still seen when splenocytes had previously been stimulated with IFN $\gamma$. 
B Cells

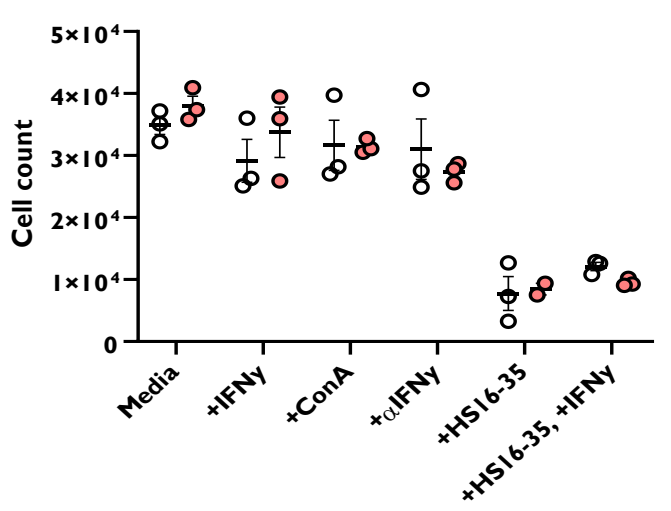

- $-\mathrm{CCL5}$

$\circ+\mathrm{CCL} 5$

Treatment Condition

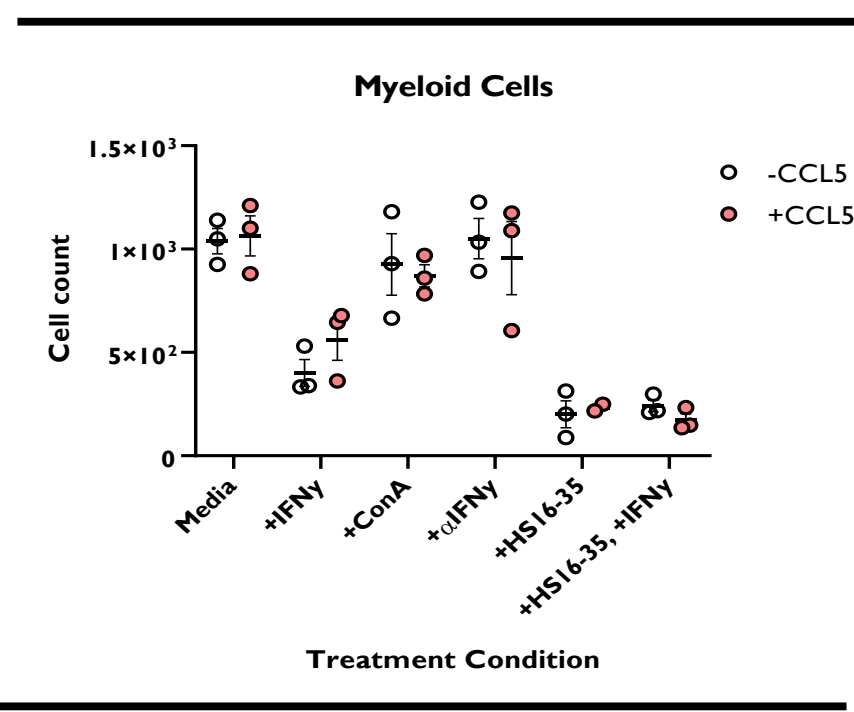

Dendritic Cells

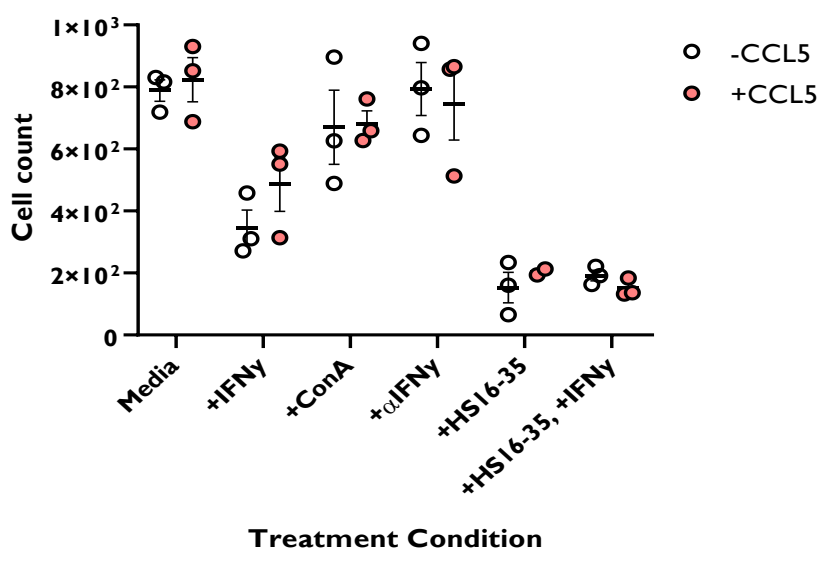

Figure 4. I0: HSI 6 reduced $B$ cell, myeloid and dendritic cell migration through a transwell model of the ChP. IFNY stimulation did not increase migration of B cells across the ECPC-4 monolayer and decreased the number of migrating myeloid and dendritic cells. In addition, ConA stimulation failed to increase migration in any of the populations shown. Regardless, HSI6-35 treatment reduced migration across the ECPC-4 monolayer from the media alone control in B cell, myeloid cell, and dendritic cell population. 


\subsubsection{HSI6-35 may be effective as an antimigratory agent by inhibiting proinflammatory cytokine release}

Because IFN $\gamma$ has previously been shown to promote migration in this model of the ChP, the research required further investigation into how HSI6-35 may modulate immune cell responses. The supernatant from splenocytes treated with or without ConA in the presence or absence of HSI6-35 was tested for IFN $\gamma$ using a sandwich ELISA, to understand if HSI6-35 is effective in this model by inhibiting proinflammatory cytokine release, in a similar mechanism to the $\alpha \mathrm{IFN} \gamma$ antibody. When stimulated with ConA alone, splenocytes produced high levels of IFNY (Figure 4. I la). as expected, however, increasing concentrations of HSI6-35 led to a significant reduction in IFNY in the culture supernatant. Given the known role for IFN $\gamma$ in promoting migration, this reduction in IFN $\gamma$ may be an alternative mechanism to heparanase inhibition by which HSI6-35 may affect immune cell migration.

To elucidate how HSI6-35 was effective at inhibiting migration of ConA-stimulated splenocytes in our model of the ChP, we evaluated the cytokines in the supernatant of the splenocyte and ECPC-4 cultures stimulated with or without ConA and/or HSI6-35 at 6 or $60 \mu \mathrm{g} / \mathrm{mL}$ for 24 hours using a LEGENDPlex ${ }^{\text {TM }}$ mouse inflammation assay. This assay allowed us to quantify 13 cytokines in culture supernatants. Additionally, we also investigated cytokine production by splenocytes from animals with EAE responded in the presence or absence of HSI6-35 to understand whether an on-going immune response altered the effect of HSI6-35 on ConA-stimulated or unstimulated splenocytes.

Four of the thirteen cytokines were upregulated by ConA in cultures from the healthy control in splenocytes: IFN $\gamma$, IL-I7A, IL-6 and TNF $\alpha$. In Figure 4. I l b, we compare the cytokine responses of EAE splenocytes to a healthy, ConA-stimulated, untreated control. In healthy splenocytes, IFN $y$ appeared to be reduced by up to $30 \%$ from the untreated healthy control with both $6 \mu \mathrm{g} / \mathrm{mL}$ and $60 \mu \mathrm{g} / \mathrm{mL}$ treatments. Interestingly, EAE splenocytes showed a more robust response, with untreated ConA-stimulated splenocytes upregulating IFNY expression by more than $600 \%$ from control. This expression was diminished back to baseline with $6 \mu \mathrm{g} / \mathrm{mL}$ treatment, and down by $400 \%$ with $60 \mu \mathrm{g} / \mathrm{mL}$ treatment.

ConA-stimulated splenocytes from EAE mice showed increased expression of IL-I7A (up to I,500\%) compared to splenocytes from healthy mice (Figure 4. I I b). While IL- I7A levels were modestly affected by $\mathrm{HSI6-35}$ in healthy splenocyte cultures, HSI6-35 reduced IL-I7A production by up to I,000\% compared to the untreated EAE splenocytes. Additionally, although the effect of HSI6-35 on IL-6 was 
highly variable in healthy splenocyte cultures, untreated EAE splenocytes elicited a strong IL-6 response (>275\% increase compared to healthy), which was reduced to levels comparable to controls with both concentrations of HSI6-35 (Figure 4.I lb). Interestingly, healthy splenocytes upregulated their expression of TNF $\alpha$ in response to increasing concentrations of HSI6-35 treatment. In contrast, EAE splenocytes expressed high levels of TNFa, which was reduced almost to healthy baseline by both $6 \mu \mathrm{g} / \mathrm{mL}$ and $60 \mu \mathrm{g} / \mathrm{mL}$ of HSI6-35 (Figure 4.I Ib). In contrast to splenocytes, ConA did not induce cytokine production by ECPC-4 cells; however, there was constitutive expression of CCL2 and IFN cells, which were increased by HSI6-35 treatment (Figure 4.I 2). 
a.

\section{Splenocyte IFNY ELISA}
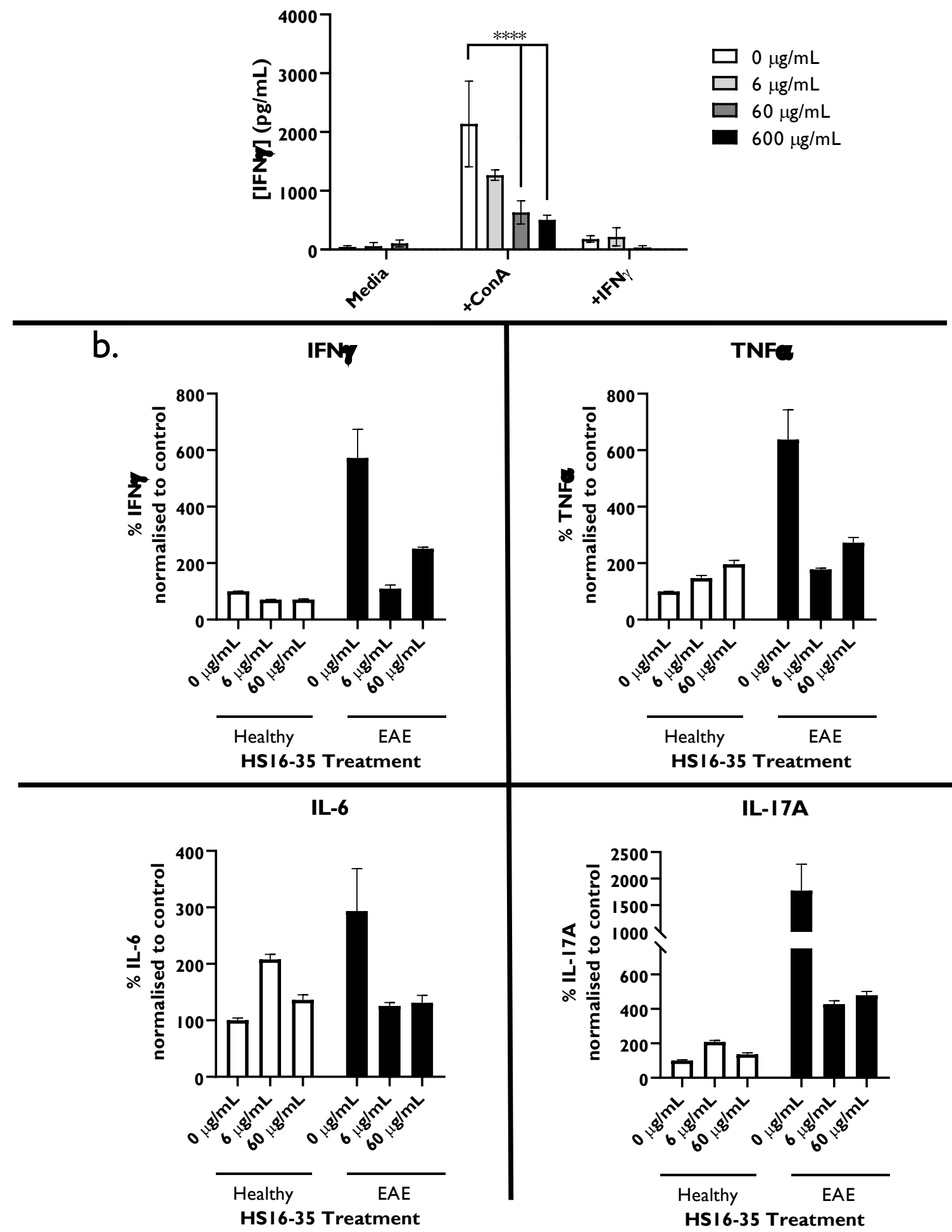

Figure 4.II: HSI6-35 mitigates proinflammatory cytokine responses from encephalitogenic splenocytes. (a) Shown are individual data points from one experiment \pm SEM. When splenocytes are stimulated with ConA, there is a significant increase in the amount of IFNY released into the media. With increasing concentrations of HSI6-35, the concentration of IFN $\gamma$ in the supernatant significantly decreases. The data from this experiment was analysed using an ordinary twoway ANOVA with $\alpha=0.05(*=p<0.05, * *=p<0.01$, *** $=p<0.001)$ (b) Shown are the means \pm 
SEM from duplicate splenocyte cultures treated with ConA for 24 hours, then resuspended in media containing HSI6-35. EAE splenocytes upregulated the expression of four key proinflammatory cytokines; IFN $\gamma$, IL-I7A, IL-6 and TNF $\alpha$, which trended towards being downregulated with increasing concentrations of HSI6-35 treatment $(\mathrm{n} / \mathrm{s})$. 


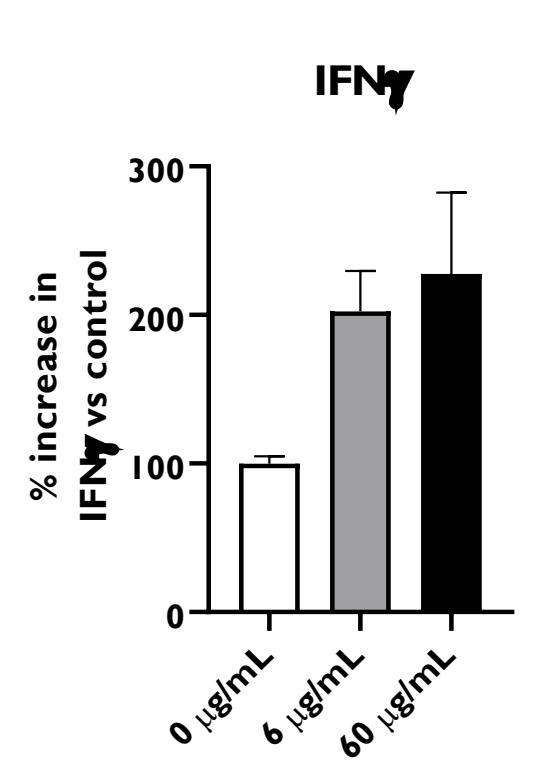

HSI6-35 Treatment
CCL2

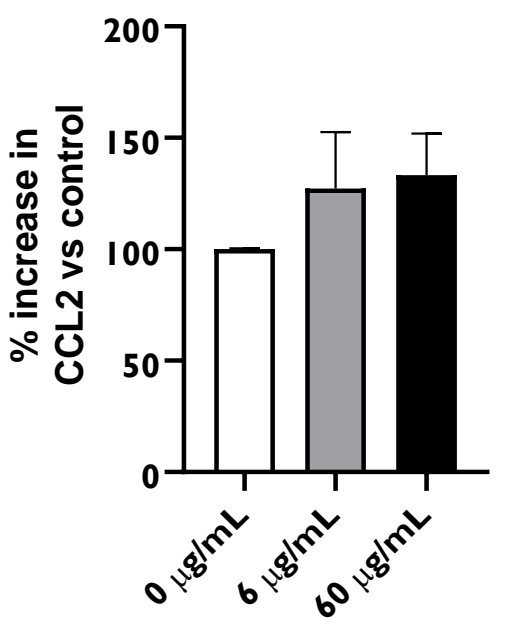

HSI6-35 Treatment

Figure 4. I 2: ECPC-4 cultures release increasing concentrations of IFNy and CCL2 which correlates with treatment concentration of HSI6-35. Shown are the means \pm SEM from duplicate ECPC-4 cultures treated with HSI6-35 for 48 hours. ECPC-4 cells expressed detectable levels of IFNY and CCLS, which trended towards being upregulated with increasing concentrations of HSI635 treatment $(\mathrm{n} / \mathrm{s})$. 


\subsubsection{HSI6-35 may be effective in inhibiting encephalitogenic leukocyte entry through a model of the ChP}

Finally, to elucidate if HSI6-35 is effective in inhibiting pathogenic migration into the CNS, we assessed the migration of leukocytes isolated from mice with EAE. EAE was induced in C57BL/6] mice as described in 2.2.3. Mice developed chronic EAE before sacrifice, and single cell leukocyte suspensions were cultured from splenic tissue as described in 2.2.5. Splenocytes were also isolated from healthy, untreated age and sex-matched controls ( $n=3$ mice per group). Splenocyte cultures were stimulated with or without ConA for 24 hours and then resuspended in fresh media containing HSI6-35 at $60 \mu \mathrm{g} / \mathrm{mL}$ for 24 hours. In parallel, ECPC-4 monolayers on transwell inserts were treated with HSI6-35 at the same concentration for 24 hours prior to the migration assay. The treated splenocytes were then added to the upper chamber and allowed to migrate for 24 hours in the presence or absence of CCL5 in the lower chamber.

This experiment was not seen to completion because of an incubator failure. This experiment would have served as a major component for obtaining biological replicates of both healthy and EAE immune cell migration, supporting the results that have already been showcased in this chapter. This project had already faced major disruptions to the development of this chapter, as Transwell inserts took 8 months to be delivered to us during the COVID-19 pandemic. This experiment used all the remaining Transwell inserts, so it could not be replicated before the conclusion of this thesis due to further shipping delays. As this was not completed, I am vitally aware that strong conclusions regarding the efficacy of HSI6-35 on migration across the ECPC-4 monolayer can be drawn yet. As many of the experiments in this chapter could only be conducted a single time, no statistical analyses are presented. For the purpose of the discussion, I will theorize about the observed differences in migration and present supporting literature, although further experimental work is required to confirm the efficacy of this compound with statistical confidence. 


\subsection{Discussion}

This chapter explores the use of novel heparan sulfate mimetic 'HSI6-35' on our previously established Transwell migration model of the ChP. We aimed to identify the effects of HSI6-35 on leukocytes isolated from healthy and EAE mice to determine how $\mathrm{HSI}$ 6-35 is protective in sick mice and how it may modulate immune responses in healthy individuals. We first adapted the optimal in vivo dose to ensure that there was no negative impact on leukocyte and epithelia viability, or monolayer permeability. We found that 60 $\mu \mathrm{g} / \mathrm{mL}(12 \mathrm{mM})$ showed no adverse effects on cells in culture, whereas higher doses showed some cytotoxicity to the epithelial monolayer. The results from the MTT and Evans blue dye permeability assays provided essential information for adapting the effective in vivo dosing regimen to an in vitro model of the choroid plexus. Indeed, Zubkova et al., (2018) found that HSI6-35 was effective in inhibiting heparanase enzyme activity, with an $\mathrm{IC}_{50}$ in the low micromolar range. We do not yet know if the unpublished in vivo data from this group reduced disease severity as a result of heparanase inhibition at the BBB or due to some other biological mechanism that has not been investigated. Considering Parish et al., 1988 and Naparstek et al., (1990) findings, it seems likely that inhibition of T-cell produced heparanase activity is the primary mechanism of action of this drug and the rationale for its success in vivo. To account for the inevitable metabolic loss of drug in the plasma in mice, versus the direct application onto to the model, we selected $60 \mathrm{ug} / \mathrm{mL}$ as the standard treatment for the transwell migration model.

Additionally, we found that at this dose, ConA and IFN $\gamma$-induced trafficking in our transwell cultures could be completely inhibited. Further investigation of the cell types impacted by HSI6-35 treatment showed that $\mathrm{T}$ cells and dendritic cells were particularly reduced however the drug showed efficacy on all major immune cell subsets including B lymphocytes and myeloid cells. Using alFNy as a control, which had previously been shown to reduce proinflammatory trafficking across the transwell culture, HSI6-35 was capable of reducing migration to a greater extent than this control.

Finally, we measured cytokine responses in isolated healthy and EAE leukocyte cultures to ConA stimulation and low/optimal doses. Many of the cytokines act as part of a tightly regulated network where the action of one cytokine is affected by the production and behaviour of other cytokines, In addition, many of these cytokines play a pathogenic or regulatory role in the development of inflammatory insult in MS. We identified four cytokines that were upregulated in response to ConA stimulation in EAE splenocytes: IFN $\gamma$, IL-6, IL-I7A and TNF $\alpha$. The expression of all of these cytokines was modulated in response to low and moderate dose HSI6-35 treatment, trending towards downregulation. 
IL-I7A is a cytokine that can be considered a ringmaster in the pathology of multiple sclerosis and EAE. IL-I7A is produced by CD4+ $T$ helper $17\left(T_{H} 17\right)$ cells, a prominent population that drives disease progression. ${ }^{126}$ In active lesions, $T_{H} 17$ cells, astrocytes and oligodendrocytes release IL-I7A which synergizes with proinflammatory cytokines and other mediators of tissue damage to drive cellular responses within the lesion. ${ }^{197-199}$ Setiadi et al., (2019) report a positive correlation between serum ILI7A concentrations and the number of relapses in MS patients ${ }^{200}$. In addition, IL-I7A has recently been shown to facilitate breakdown of the blood-brain barrier in RRMS. A breakthrough study by Huppert et al., (20I0) showed that brain endothelial cultures co-treated with IL-I7A and IL-6 downregulated genes coding for proteins associated with BBB integrity; occludin (OCLN), zonula occludens-I (ZO-I), VEcadherin and platelet endothelial cell adhesion molecule (PECAM-I), resulting increased cellular permeability of the in vitro cultures. ${ }^{201}$ In addition, ILI7A-/- mice or mice treated with i.p. alL-I7A before disease onset show less disruption of BBB integrity and lower disease scores. ${ }^{201,202}$

IL-6 is another cytokine with a vital role in EAE and MS. IL-6 is a key component of the acute phase response and is secreted by lymphocytes, macrophages, microglia and some types of non-immune cells such as endothelial cells and neurons. ${ }^{203}$ Hand-in-hand with IL-I7A, IL-6 aggravates the clinical manifestations of MS through dual actions in the CNS and periphery. IL-6 promotes the generation of $\mathrm{T}_{\mathrm{H}} 17$ type cells in the peripheral lymphoid tissues, which are known to play a major role in the immune assault against CNS antigens, perpetuating the neuroinflammatory response. ${ }^{204}$ In patients with active RRMS, IL-6 has been shown to promote the resistance of effector T cells to regulation by Tregs. 205 Recently, Erta et al., (2016) showed that in female mice with astrocytic IL-6 deficiency, there was a modest amelioration of EAE pathology as seen through later onset of clinical symptoms, fewer inflammatory infiltrates, and reduced demyelination from controls. ${ }^{206}$ IL-6 responses in MS patients are upregulated in the sera and CSF by up to 17 times that of healthy controls. Because IL- 6 drives immunoglobulin responses from B cells, systemic upregulation of IL-6 in MS supports a role for B cells in disease pathology. ${ }^{207}$ Overall, the literature highlights a definite role for IL-6 in the pathogenesis of EAE and MS.

TNF $\alpha$ has been a suggested target for MS therapies for decades now. TNF $\alpha$ is a pleiotropic cytokine with many homeostatic roles as well as known pathological roles in diseases such as rheumatoid arthritis, irritable bowel syndrome and psoriasis. ${ }^{208}$ Many of these diseases show modest responses to antiTNF $\alpha$ therapies. Because TNFa signals through dual pathways based on its receptor-ligand complex formation, either TNFRI or TNFR2, a preference towards TNFR2 signalling may have a protective effect in MS. 208 
Indeed, an in vivo study has shown the dichotomy between TNFRI and TNFR2's roles in EAE. TNFR I-1mice show reduced clinical manifestations of EAE symptoms, lower $\mathrm{CD} 3^{+} \mathrm{T}$ cell infiltrates and activated microglia/macrophages versus wildtype mice. In contrast, TNFR2-/- mice have worsened disease course. ${ }^{209}$ TNFR2 also plays a neuroprotective role during remyelination by protecting oligodendrocyte precursor cells (OPC's) from oxidative stress and boosting OPC maturation through TNFR2-stimulation in astrocytes. ${ }^{210,211}$ Because of TNFa's dual roles in MS, it is difficult to suggest if altering its expression will be protective or detrimental.

Previous literature has elucidated that ConA stimulation of murine splenocytes induces a wave of IFN $\gamma$ signalling. ${ }^{212}$ IFNY is a cytokine with dual roles in MS, it is heavily upregulated in lesion areas and is associated with a destructive $T_{H} I$ response in the CNS.213 Indeed, in both MS and EAE models, IFNY administration is associated with increased relapse rate and severity of symptoms. ${ }^{184,213}$ Skewing the cytokine milieu and immune response to $T_{H} 2$ in EAE clearly ameliorates symptoms of the disease. ${ }^{214}$ In stark contrast, IFN $\gamma$ is also suggested to have a beneficial role by limiting the extent of disease, as seen by increased disease severity in IFN $\gamma$ knockout mice. ${ }^{215}$ In EAE, IFN $\gamma$ may also enhance oligodendrocyte survival, which is associated with reduced disease severity and lesser demyelination than vehicle treated controls. 216

Because many of these cytokines have been identified as having dual roles in disease pathology of MS, it could be suggested that regulating, not completely inhibiting the $T_{H} I$ and $T_{H} I 7$ cytokine responses in vitro may be protective in EAE and MS.

Focusing on the choroid epithelium, we saw increasing concentrations of CCL2 and IFNY expression which correlated with HSI6-35 concentrations in our ECPC-4 cell line. Interestingly, Robichon et al., (2020) showed that CCL2 secretion is associated with pathogenic macrophage migration into the CNS in EAE and when inhibited, protects against EAE by reducing cell infiltrates into the CNS. ${ }^{152}$ However, CCL2 gene is highly expressed in the basal state of the choroid plexus, compared to other CNS tissues, which may be reported in this assay, with some variability. ${ }^{217}$ Shifts in IFN $\gamma$ expression in the choroid plexus have been reported in aging-associated cognitive decline and Alzheimer's disease but expression is also important in regular immune surveillance and repair, reported by Kunis et al., (2013). 119,161,218

MS has classically been considered a T-cell mediated disease, with studies showing that relapses are predominantly mediated by an aberrant and unregulated pro-inflammatory effector $\mathrm{T}$ cell response, 
encompassing both $\mathrm{CD}^{+}$and $\mathrm{CD} 8^{+} \mathrm{T}$ cells that traffic into the neural parenchyma. ${ }^{46}$ These cells express receptors for CNS antigens, and complex interplay between them and CNS-resident cells lead to the production of inflammatory mediators that can recruit further inflammatory cells into the CNS to induce neuroaxonal damage and neurodegeneration. Reboldi et al., (2009) elucidated how the choroid plexus acts as the initial site of entry for $T_{H} I 7$ cells in EAE, which induced a secondary wave of inflammatory cell infiltration through the now-permeable cerebral vasculature. ${ }^{123}$ Interestingly, we saw that with ConA stimulation, there was higher numbers of $\mathrm{CD} 8^{+} \mathrm{T}$ cells than $\mathrm{CD} 4^{+} \mathrm{T}$ cells, particularly of naive phenotypes crossing the $\mathrm{ChP}$ monolayer. This is in line with supporting literature, which indicates that $\mathrm{CD} 8^{+} \mathrm{T}$ cells are the predominate lymphocyte present in the lesions of patients with MS. ${ }^{219,220 ~ I t ~ w a s ~ u n u s u a l ~ h o w e v e r, ~}$ that $C D 8^{+} T_{N}$ cells were actively penetrating the epithelial monolayer given that naïve cells generally do not express the correct chemokine receptors or adhesion molecules to do so in vivo. It could be postulated that this is a result of bystander activation, in which ConA stimulated CD4+ $\mathrm{T}$ cells undergo activation and clonal expansion in an Ag-independent manner, releasing high concentrations of proinflammatory cytokines into the supernatant. Although the mechanism behind this phenomenon is unknown, type I interferons have previously been reported to induce functional activation in CD8 ${ }^{+} \mathrm{T}$ cells which remain in a $\mathrm{T}_{\mathrm{N}}$-type phenotype. ${ }^{221}$

We observed very low numbers of $\mathrm{CD}^{+}$naïve $\mathrm{T}$ cells penetrating the monolayer, in both unstimulated and ConA-stimulated cultures, as they likely do not express the correct adhesion molecules or activation markers to actively migrate through the monolayer. Because we know that similar trafficking patterns happen in vivo, this was indicative once again that the ECPC-4 monolayer was a selective, yet permeable barrier similar to the $\mathrm{ChP}$ in the brain. Both central memory $\mathrm{T}$ cell and effector memory $\mathrm{T}$ cell trafficking across the monolayer was upregulated with ConA stimulation, which was significantly reduced in both conditions when treated with HSI6-35. CD8 ${ }^{+}$T cells generally dominate the early MS infiltrates, and are more abundant in both forms of progressive MS in the CP itself. ${ }^{17,222}$ In our model of the ChP, we saw that HSI6-35 targeted these key pathogenic populations, namely CD4 ${ }^{+} T_{E M}$ and $T_{C M}$ and $C D 8^{+} T_{N}, T_{E M}$ and $\mathrm{T}_{\mathrm{CM}}$ lymphocytes. By regulating entry of these cells across the ChP, some of the initial inflammatory events that establish MS disease progression could be arrested before symptom onset.

In addition, MS pathology occurs as the result of insufficient tolerance mechanisms, and the resistance of effector T cell groups to Treg-mediated regulation. Koliesnik et al., (2020) reported that heparan sulfate mimetic 'PG545', inhibits the Erk I/2 signalling pathway that drives $T$ cell activation and polarization. They 
also report that through this mechanism, effector $T_{H} I$ and $T_{H} I 7$ responses are suppressed in preference of FoxP3+ Treg subsets both in vitro and in an in vivo DHT model. ${ }^{144}$ Similarly, if our heparan sulfate mimetic is capable of skewing the immune response in favour of Treg responses in the neural parenchyma, $T_{H} I$ and $T_{H} 17$ responses in the ChP and CSF may be sufficiently regulated and unable to elicit CNS injury. This suppression may be evidenced through the reduction of key $T_{H} I / T_{H} I 7$ cytokines in our Transwell system when treated with HSI6-35, although this requires more investigation. Finally, ConA-induced $\mathrm{T}$ cell heparanase expression has reportedly been inhibited with HS-like compounds, as described by Lider et al., (1990). Inhibiting heparanase in vivo has been reported to be protective in EAE, by mitigating the enzyme's capacity to destabilize the ECM and surrounding vasculature and inhibiting its proadhesion properties in sheer flow environments. ${ }^{138,223}$ The multiple effects of heparan sulfate mimetics on CNS infiltration, alongside our in vitro data show that HSI6-35 may exert a regulatory effect that is effective in protecting against disease in EAE and MS.

In MS and EAE, the infiltration of autoreactive $T$ lymphocytes, macrophages and demyelinating antibodies into the CNS is one of the hallmark features of disease development. ${ }^{17}$ Inhibiting the trafficking of early peripheral cell infiltrates has proved to be one of the most successful treatment options for MS patients, evidenced by frontline drugs such as natalizumab.224 A major caveat of these drugs is they provide complete inhibition of immune cell trafficking, regardless of whether the cells are performing routine immune surveillance or pathogenic inflammation. This provides significant risk to patients, more so if they are $\mathrm{CSF}^{+}$for the $\mathrm{JC}$ virus as these patients can develop life-threatening PML due to defective immune surveillance in the CNS.62 Interestingly, HSI6-35 did not completely suppress cell migration through the transwell culture, but reduced trafficking to near baseline healthy levels. This suggests that HSI6-35 may restrict but not indiscriminately suppress immune cell trafficking into the CNS in a pathogenic model. Together, this chapter provides major groundwork to elucidate how HSI6-35 acts in vivo and contributes to disease protection in EAE. 


\subsection{Summary}

This chapter investigated the effect of novel HS-mimetic, HSI6-35, on the migration of immune cells through a model of the ChP. HSI6-35 exerted no cytotoxic effects on splenocytes isolated from healthy mice or mice with EAE. It showed limited toxicity on ECPC-4 cells and did not disrupt overall barrier function of the epithelial monolayer. Using the transwell migration model, we found that the HS mimetic significantly reduced the number of cells that migrated through the monolayer, in both homeostatic and proinflammatory conditions. Specifically, HSI6-35 appeared to be most effective in inhibiting ConAstimulated $\mathrm{CD}^{+}$and $\mathrm{CD} 8^{+} \mathrm{T}$ cell migration but showed broad inhibition of homeostatic migration across all CD45+ leukocytes. Finally, we found that four key proinflammatory cytokines, IL-6, IFN $y$, TNF $\alpha$ and IL17A were downregulated in EAE splenocytes stimulated with ConA in the presence of HSI6-35 in vitro. Taken together these findings suggest that the mechanism, by which HSI6-35 inhibits migration in our $\mathrm{ChP}$ model, involves regulating cytokine (esp. IFN $\mathrm{Y}$ )-induced migratory signals and may not be solely by inhibiting heparanase-mediated ECM degradation. 
Chapter 5: General Discussion 


\section{I. Overview}

Multiple sclerosis is a chronic, neurodegenerative disease characterised by confluent areas of demyelination in the white and grey matter of the brain and spinal cord. These areas of sclerotic damage encompass the loss of myelin sheaths and death of oligodendrocytes and in the latter stages, neuronal death. Inflammatory damage to the neuronal parenchyma is considered to be of autoimmune origins, but no definitive aetiology of MS has been discovered. The inflammatory migration of leukocytes into the CNS is one of the key features of MS, with early lesions showing peripheral infiltrates and breakdown of the blood-brain barrier. ${ }^{17}$

Inhibiting the infiltration of these cells has been the main target of several frontline therapies. Although these drugs successfully inhibit immune cell trafficking into the CNS, decreasing disease severity and relapse rate, they are often associated with severe and sometimes life-threatening side effects. ${ }^{59}$ Because of their broad-spectrum immunomodulatory actions, they do not distinguish between immune cells conducting homeostatic surveillance or pathogenic infiltration, leading to immune dysfunction in the CNS. Unchecked, pathogens in the CNS can freely exert their effects, completely unregulated to cause CNS injury. Hence, there is an unmet need for treatments that regulate, not inhibit trafficking into the CNS.

Although the CNS was traditionally considered an immune privileged site, recent studies have illuminated that homeostatic trafficking of leukocytes for immunosurveillance through the ventricles is governed by the choroid plexus. In contrast to the blood-brain barrier, in which trafficking through this structure constitutes a hazard to the tissue, the choroid plexus accommodates over 500,000 cells trafficking through the ventricles each day, each performing an important role in maintaining the immunosuppressed phenotype inside the CSF.168 Interestingly, during homeostatic functions and even sterile injury, the choroid plexus releases a slew of immunomodulatory mediators which push the immune cells in its immediate vicinity and CSF into a pro-resolving, wound-healing phenotype. ${ }^{37}$ In MS, there is emerging evidence to suggest that the choroid plexus contributes to disease pathology. Indeed, there are significant enhancements in choroid plexus anatomy of MS patients, documented on MRI by Kim et al., (2020).225 In addition, the ChP houses a small population of resident dendritic cells, who have been reported to conduct antigen sampling in the CNS." in the choroid plexus. ${ }^{166,226}$ Depending on the inflammatory or tolerogenic state that $\mathrm{DC}$ in the ChP adopt, the ChP has immense capacity to govern the type of response entering the CNS. These DC and other stromal macrophages express high levels of MHC II on their surface, indicating that the ChP acts as an 
interphase for constitutive T cell stimulation and reactivation in CNS inflammatory disorders. ${ }^{20,125,168,227}$ Indeed, the $\mathrm{ChP}$ integrates and responds to various signals from the peripheral immune system, including inflammation. In response to peripheral cytokine stimulation, the ChP upregulates key adhesion molecules, indicating that it may facilitate inflammatory trafficking into the CNS.121 Inhibiting the expression of these molecules, has shown to be effective in protecting from EAE, as described in Breuer et al., (20I8) where inhibiting the interaction of laminin 4II:MCAM in the ChP restricted encephalitogenic transmigration in an in vitro model of $\mathrm{ChP}$ endothelium and in EAE mice. ${ }^{228}$

The requirement for a working in vitro model for the study of migration into the CNS is clear, and something the current study addresses. Only one other study reports the development of a mousederived ChP for the study of leukocyte trafficking, using primary mouse ChP cells. ${ }^{172}$ To reduce the number of animals whilst troubleshooting, we used an immortalised ChP epithelial cell line; ECPC-4. Although Munechika et al., (1996) report that this cell line maintains characteristic feature of the ChP epithelium such as transthyretin and $\alpha$-macroglobulin, Lazarevic et al., (2016) raise concern over the capacity of this cell line to model functions of the ChP, namely the expression of adhesion molecules and its ability to form a tight barrier with contact-inhibition. This thesis demonstrated that in culture, ECPC4 cells formed complete monolayers with tight barrier functions and were suitable for use in the Transwell migration assay.

In addition, we observed that inflammatory trafficking of leukocytes across the ECPC-4 monolayer could be induced by ConA or IFNY stimulation. ConA has previously been shown to increase trans-endothelial T cell migration in a model of general vasculature. ${ }^{229} \mathrm{In}$ addition, $\mathrm{T}_{\mathrm{H}} \mathrm{I}$-type cytokines such as IFN $\gamma$ or TNF $\alpha$ have been shown to increase CD4+ $\mathrm{T}$ cell migration across this model.229 Our findings remain consistent with this literature, in which we observed T-cell specific increases in migration with stimulation of both ConA and IFNY.

The literature offers a clear role for heparanase, the ubiquitously expressed heparan sulfate degrading enzyme in many neuroinflammatory diseases, including MS. It has been extensively studied in cancer research for its ability to fragment HS residues from their proteoglycan core, leading to ECM remodelling, the propagation of cell signalling, metastasis, and leukocyte migration. ${ }^{135}$ Some of the initial work focusing on the role of heparanase in MS investigated T cell heparanase release in the cerebral vasculature, which facilitated inflammatory trafficking across the destabilised BBB. ${ }^{137}$ The current study focused on novel heparan sulfate mimetic HSI6-35, part of a targeted library of novel compounds developed by the Zubkova 
lab at the Ferrier Research Institute. Because HSI6-35 is comprised of a dendritic core and heavily sulfated maltose groups, its synthesis is much quicker, making it a commercially available cheaper alternative to other HS-like compounds currently in clinical development. ${ }^{145}$ We investigated the capacity of HSI6-35 to mitigate ConA and IFN $\mathrm{Y}$-mediated migration across our transwell model of the choroid plexus. With HSI6-35 treatment, we saw suppression of CD45+ leukocyte migration to baseline levels (chapter 4), which may provide a protective effect in vivo.

Controversially, there are a limited number of studies that suggest heparanase, or displacing interactions with its cognate ligand, HSPG's may provide a protective effect in the EAE model of MS. Harris et al., (2016) describe that displacing heparanase's endogenous interactions with HSPG's by small molecule inhibitors was protective in EAE. I.P. injection of these compounds was effective in reducing the appearance of symptoms and inflammation during disease progression. The authors ascribe this to the direct inhibition of binding to heparan sulfate, which blocked leukocyte rolling in the vasculature and subsequent migration into the CNS. ${ }^{230}$ This defends against the notion that heparanase activity, in and of itself is pathogenic in EAE. In addition, Muramtatsu and Kadomatsu., (2014) describe how midkine, a small cytokine that binds highly sulfated proteins can influence the disease course of EAE. By removing midkine using RNA aptamers, Takeuchi et al., (2014) found that EAE progression was suppressed by expansion of Tregs and DCregs and suppression of $T_{H} l / T_{H} I 7$ polarisation. ${ }^{23 l}$ Because midkine so heavily associates with HSPG's, and it's removal has been shown to be beneficial, this suggests that heparanase activity, through cleaving HS residues would reduce binding sites for midkine, offering a similar advantage in reducing leukocyte trafficking and increasing Treg numbers. ${ }^{231,232}$

Subsequently, studies have shown that the sulfated proteoglycans; chondroitin sulfates are heavily expressed by astrocytes and microglia. During brain injury, several studies have shown that chondroitin sulfates proved a hostile environment that impedes OPC mobilization and remyelination, as treatment with chondroitinase is beneficial on OPC recruitment in vivo. $233-235$ In vitro, highly sulfated proteoglycans have been shown to delay OPC maturation which is expected to delay the remyelination response in vivo. ${ }^{236}$ Inhibiting the synthesis of CSPG's was also found to accelerate OPC maturation and remyelination in a murine model of focal demyelination. 237 This suggests in part, that the activity in heparan sulfatedegrading enzymes such as heparanase and chondroitinase may prove beneficial in providing a matrix for migrating OPC's to reach the lesion site and accelerate recovery. Only one study has administered heparanase to EAE animals as a therapeutic effort. Bitan et al., (2010) showed that I.P. heparanase 
administration ameliorated clinical signs of EAE in a dose-dependent manner. This protection was associated with inhibition of splenocyte proliferation and the modulation of cytokine responses in favour of a $T_{H} 2$ response. ${ }^{238}$ Although this study suggests that heparanase may be protective in EAE, the therapy administered was recombinant heparanase, with loss-of-function mutations so it was unclear if its protective effects were due to fragmenting HSPG's.

On the other hand, there is an extensive list of literature that discusses the potential detrimental role for heparanase in EAE and MS. Much of this discusses its role in breaking down the tight barrier function of the BBB and ChP, which we discussed further in chapter 4. Consistent with our findings in vitro, IronyTur-Sinai et al., (2003) showed that synthetic heparin mimetic RG-I3577 ameliorated EAE, reducing clinical symptomology and reducing inflammation in the spinal cord. In vitro, RG-I3577 impaired leukocyte trafficking mechanisms, reduced $\mathrm{T}$ cell heparanase expression and interestingly, astrocytic heparanase expression related to its activity against Erkl/2 phosphorylation.239 These findings are similar to Koliesnik et al's., (2020) experiment showing that another heparan sulfate mimetic, PG545 inhibits ErkI/2 phosphorylation, supressing TH cell polarization and proliferation. ${ }^{144}$ If our mimetic HSI6-35 acts through this pathway in vivo, it may be able to regulate $T_{H} I / T_{H} 17$ polarisation in favour of Treg regulation, although this must be investigated further. Early evidence from our cytokine assays, in which HSI6-35 appears to downregulate key $T_{H} I / T_{H} I 7$ cytokines and promote a naïve T cell phenotype may indicate that HSI6-35 acts in a similar mechanism. In addition, the ConA mediated increase in total CD8+ $T_{E}$ and $T_{C M}$ cell numbers appears to be reduced when splenocyte cultures were treated with HSI6-35, evidence which further points to the suppression of T cell proliferation.

Heparanase has previously been shown to modulate the interactions between APC and T cells by digesting HSPG's on the surface of APC's. Two studies by Chung et al., (2007, 20I4) using isolated murine splenocytes demonstrate that a specific receptor; the dendritic-cell associated HSPG dependent integrin ligand (DC-HIL) can inhibit T-cell activation by binding syndecan-4 on $T_{E M}$ cells. ${ }^{240,24 I}$ If heparanase is present, it may inhibit the interaction between DC-HIL and its cognate ligand by fragmenting HS side chains on syndecan-4, allowing T cell activation and proliferation. By suppressing heparanase through this mechanism, T cell proliferation could be supressed which may be protective in EAE and MS. Further work to investigate these interactions and how they may be altered by HSI6-35 stimulation is needed.

In addition to leukocyte-derived heparanase in the periphery, there are multiple roles for heparanase inside the CNS in both EAE and MS. Heparanase has been shown to be upregulated in the colonic 
epithelium of patients with inflammatory bowel disease, a chronic inflammatory condition. ${ }^{242}$ Because the mucosa responds to chronic inflammation by upregulating heparanase, it is suggested that in the ChP epithelium, similar expression of heparanase could occur during chronic inflammatory insult. Inside the CNS, van Horssen et al., (2006) identified that heparan sulfate distribution in active lesions of MS patients was discontinuous and irregular, which was not identified in the neural parenchyma of chronic inactive lesions or healthy controls. This suggests that in part, heparanase activity in the CNS may facilitate the development of lesions by digesting the ECM and facilitating further recruitment of pro-inflammatory leukocytes and mediators. ${ }^{243}$ Targeting heparanase within the CNS is a future goal of this project. 


\subsection{Limitations and future directions}

The current study was limited in several areas, namely obtaining biological replicates for a number of experiments. One of the major impacts on research efficacy was a significant delay in shipping Transwell inserts due to the COVID-19 pandemic (8 months); so that we only had the time and materials to conduct a limited number of migration experiments with the HSI6-35 heparan sulfate mimetic before the conclusion of this thesis. In two separate models of proinflammatory trafficking (IFNY mediated and ConA mediated), the HS-mimetic was capable of reducing migration, and although these are not replicates, the results show a general trend that this compound may be effective. In terms of interpreting the results of this study, this limits our capacity to draw any major conclusions about the efficacy of HSI6-35 on migration through a model of the choroid plexus, although this work will be continued farther in a doctorate degree.

A major caveat of this study was that we were not able to test HSI6-35 on encephalitogenic splenocytes isolated from animals with EAE. In our cytokine analysis, we saw that EAE splenocytes untreated with our heparan sulfate mimetic produced a much greater cytokine response, that could be ameliorated with HSI6-35 treatment. Because T cell heparanase response is also associated with the encephalitogenicity of invading lymphocytes and is upregulated during EAE, it is likely that we may see a greater impact on migrating leukocytes when heparanase expression is inhibited. ${ }^{140}$ This experiment was planned and conducted in triplicate, but due to an incubator failure, this could not be seen to completion. This is the definitive next step for the continuation of this project. In addition to this, future experiments could involve transitioning to cultures of primary choroid plexus cells from healthy animals and EAE animals as our monolayer. This may provide a better replication of the in vivo state of this interface in both health and disease.

The Transwell cultures themselves were somewhat limited in mimicking the ChP in vitro. Primarily, this is due to the model being 'unifactorial', i.e., we only utilised one key chemokine identified as a major player in cell infiltration in MS and EAE: CCL5. In future experiments, the model could be upgraded to better reproduce the inflamed CNS by using other cytokines and chemokines upregulated in the CSF of MS patients. For example, CCL2 has been identified as another key chemokine that acts as a myeloid chemoattractant protein in EAE, whereas CCL5 does not. When CCL2 was supressed, Robichon et al., (2020) found that there was decreased monocyte infiltration into the CNS of EAE animals. ${ }^{152}$ By including chemokines like CCL2, a population of cells not affected by CCL5 stimulation can be targeted. For these 
cultures, we could establish an 'EAE media' of sorts, which provide leukocytes in culture with essential inflammatory mediators that more accurately mimic the environment inside the CNS of an EAE animal. In addition, a logical next step for this project would be to assess the expression of various chemokine receptors. During MS pathology; CCL2, CCL3, CCL4, CCL5, CCL7, CCL8, CXCL9 and CXCL10 have all been identified at lesion sites and reported in the CSF of patients experiencing active relapse at levels significantly higher than patients with non-inflammatory neurological disease. 150,244,245 To assess which chemokines healthy versus encephalitogenic cells respond to, a panel of these cytokines could be applied, with flow cytometry to assess the expression or upregulation of the receptor complex.

In a similar manner, other immune cell stimulants could be utilized to study the migration of various subpopulations across our monolayer. For example, B cells have recently been identified as a pathogenic population in MS disease progression. ${ }^{67}$ Although, it is not until the latter stages of disease that $B$ cells enter the CNS, where they reside in tertiary lymphoid structures in the CNS and contribute to disease pathology. To study the effects of B cell migration into the CNS in the late-stage MS, a B-cell specific stimulus could be applied. For example, LPS stimulation of splenocyte cultures leads to polyclonal B cell activation, clonal expansion and the expression of inflammatory cytokines; IL-I, IL-6, IL-8, and TNF- $\alpha$ in a similar manner to ConA stimulation of T cells. ${ }^{246}$ This would allow us to assess the capacity of HSI6-35 to regulate inflammatory $B$ cell trafficking across the choroid plexus in the context of EAE, which has not previously been elucidated.

One of the issues that we experienced with ConA or IFNy stimulation of healthy splenocyte cultures was variability of the response. The appendix in chapter 3 elucidates how three different experimental replicates induce vastly different degrees of migration across our ECPC-4 monolayer. Although each experiment showed that ConA upregulated migration across the monolayer, the extent and significance varied greatly. This may be general variation due to external factors such as animal age or health, however it may also be representative of lab technique, as the difference in migration appeared to increase and become clearer with each replicate.

Interestingly, Stone et al., (2019) incorporate cultures of astrocytes, pericytes and microglia in the CNS compartment of their BBB transwell model. Future directions for this project include the co-culture of either resident stromal cells of the ChP or resident CNS immune cells such as microglia, which secrete soluble factors and provide key cell-cell interactions that will more accurately mimic this tissue and the 
response of immune cells in vitro. Dysregulation of these cells has been documented in EAE and MS so will provide a more dynamic aspect to the system. ${ }^{247}$

It may prove beneficial to isolate supernatant from the upper and lower wells of our transwell assay following the experiment's completion to assess how the cytokine responses differ in cells that successfully navigate the $\mathrm{ChP}$ epithelium versus those who did not migrate. Similarly, creating a flow cytometry staining panel that allows us to look at $T_{H}$ cell subsets will allow us to assess whether HSI6-35 skews $\mathrm{T}$ helper cell differentiation in favour of $T_{\text {reg }}$ subsets versus $T_{H} I / T_{H} I 7$ responses as previously described in the literature.

To understand the mechanisms by which HSI6-35 is acting on both our ChP monolayer and leukocytes in culture, future experiments could investigate mRNA and protein expression. For the ECPC-4 or primary choroid epithelium, this would focus on whether the HS-mimetic downregulates expression of adhesion molecules or tight junction molecules, particularly in cell cultures that have been co-cultured with activated, proinflammatory leukocytes. This would assess the capacity of HSI6-35 to overcome the effects of proinflammatory cytokines in the media on the epithelial cells, which has previously been shown to upregulate adhesion molecules and increase the permeability of the ChP. ${ }^{248}$ For both immune cells and the epithelia, we will investigate whether heparanase expression is altered, to assess whether cells upregulate transcription when heparanase in the ECM is inhibited in a feedback loop. Additionally, the intracellular pathways that HSI6-35 may regulate have not been elucidated, but literature suggests that the MAP kinase pathway, specifically Erkl/2 phosphorylation may be at play. Using western blotting techniques, we aim to discover the intracellular mechanisms of HSI6-36.

We have not yet elucidated the mechanism by which HSI6-35 exerts its regulatory effects on migrating leukocytes. To assess if this regulation lies with inhibiting heparanase, or in suppressing proinflammatory cytokine release, we will use splenocytes from heparanase ${ }^{-/}$mice in the transwell model of the ChP and BBB. If there is a significant difference in migration across the monolayer with the knockout splenocytes, we can assess the amount that heparanase inhibition contributes to migration, and if this is a synergistic effect with inhibiting proinflammatory cytokine release. Gutter-Kapon et al., (2016) performed a similar experiment, mimicking the tumour microenvironment with heparanase ${ }^{-/}$macrophages. When heparanase expression was abolished, cell motility and phagocytosis was significantly reduced, and proinflammatory cytokine release was inhibited. This indicates that heparanase is a critical component of macrophage activation and recruitment. 249 
To understand if HSI6-35 will be clinically translatable, we will test the capacity of the mimetic against commercially available migration inhibitors for MS, particularly Natalizumab in the transwell migration model. Hopefully, at this time we will also have established an in vitro working model of the BBB, where we can additionally assess HSI6-35's activity against Natalizumab. A major research goal for this project is to show that HSI6-35 regulates, not completely ablates cellular migration into the CNS. This provides a platform for functional immunosurveillance to continue, while pathogenic lymphocytes and their mediators are unable to enter the CNS to exert their effects. A major factor contributing to the dangerous side effect profile of other migration inhibitors is the potential for PML due to the indiscriminate inhibition of lymphocyte entry into the CNS. ${ }^{62}$ We hope to show that HSI6-35 targets particular subsets of immune cells, suppressing their effector functions and capacity to migrate into the CNS. Indeed, preliminary evidence suggests that HSI6-35 exerts its effects primarily on T-lymphocytes. This can then be transitioned into animal research, using EAE mice to further develop the drug for clinical use.

Both progressive forms of MS are characterized by well established, chronic inflammation in the CNS, with a fading number of cells actively migrating in. At these stages, there is notable ventricular enlargement with brain atrophy and demyelination in the cortical matter as a result of tertiary lymphoid structure development. ${ }^{17}$ Diffuse demyelination throughout the brain is an excellent target for therapies that promote OPC recruitment and maturation, which in turn promotes remyelination and repair of the damaged tissue. ${ }^{250}$ Although our transwell model lacks the capacity to study remyelination capacity of HSI6-35, a transition to a mouse model will allow us to assess this. The cuprizone model is a diet-mediated inducer of demyelination in the corpus callosum, also leading to oligodendrocyte death. A recent study by Macchi et al., (2020) showed that the expression of genes related to HS-production in oligodendrocyte precursors were upregulated following demyelination, which was inversely correlated with lesion area and positively correlated with the remyelination potential. In addition, the study also reports that the expression of HSPG's by this population modulates the response of microglia in favour of an M2 phenotype, promoting healing responses. This suggests that HSPG's in the brain may perform important roles in the CNS following injury, and if HSI6-35 enters the CNS, it could exert positive effects on lesion resolution. ${ }^{251}$ If this is possible, the capacity for repair could be applied to other neuroinflammatory conditions such as neuromyelitis optica spectrum disorders, in which demyelination and neuronal damage occurs as a consequence of astrocytic damage and is fatal in one third of patients. ${ }^{252}$ 
In conclusion, this research has made significant headway in developing a working transwell model of the choroid plexus using immortalised choroid plexus epithelial cells. We showed that the model could reproduce inflammatory leukocyte trafficking across the inflamed ChP. In addition, IFN $\gamma$ was identified as a key factor that mediated trafficking across this structure. A potential mechanism for HSI6-35, a heparanase inhibitor was identified by cytokine analysis; suppressing the proinflammatory cytokine release associated with EAE leukocytes. Further work is required to assess if this is dependent on inhibition of the MAP kinase pathway associated with T cell activation and proliferation. Finally, the work in this thesis showed that both ConA-dependent and IFNy dependent migration could be ameliorated with HSI6-35 treatment. This suggests that treatment with HSI6-35 may be effective at ameliorating disease in EAE splenocytes, and potential in MS patients. As with every scientist researching MS, it is our hope to one day provide patients with a safer, more effective treatment to manage their MS so that they feel like more confident, independent individuals in their daily lives. 



\section{LITERATURE}

All figures in this thesis were created using BioRender.com, and all graph/chart elements were created using Prism v8.0.

I. Marshall, J. S., Warrington, R., Watson, W. \& Kim, H. L. An introduction to immunology and immunopathology. Allergy, Asthma Clin. Immunol. I4, I-I0 (2018).

2. Murphy, K. \& Weaver, C. Janeway's Immunobiology. (W.W. Norton \& Company, 2016). doi: $10.1201 / 978 \mid 315533247$.

3. Turvey, S. E. \& Broide, D. H. Innate immunity. J. Allergy Clin. Immunol. I25, S24-S32 (2010).

4. Bonilla, F. A. \& Oettgen, H. C. Adaptive immunity. J. Allergy Clin. Immunol. I25, S33-S40 (20I0).

5. Alberts, B. et al. B cells and Antibodies. in Molecular Biology of the Cell (2002).

6. Crotty, S. A brief history of T cell help to B cells. Nat. Rev. Immunol. I5, I85-189 (2015).

7. Moran, A. E. \& Hogquist, K. A. T-cell receptor affinity in thymic development. Immunology I35, 26I-7 (20I2).

8. Starr, T. K., Jameson, S. C. \& Hogquist, K. A. Positive and negative selection of T cells. Annu. Rev. Immunol. 2 I, I39-I76 (2003).

9. Weninger, W., Manjunath, N. \& Von Andrian, U. H. Migration and differentiation of CD8 + T cells. Immunol. Rev. 186, 22I-233 (2002).

10. Zhang, N. \& Bevan, M. J. CD8+ T Cells: Foot Soldiers of the Immune System. Immunity 35, I6I$168(201 \mathrm{I})$.

II. Huseby, E. S., Huseby, P. G., Shah, S., Smith, R. \& Stadinski, B. D. Pathogenic CD8 T Cells in Multiple Sclerosis and Its Experimental Models. Front. Immunol. 3, (20I2).

12. Murphy, K. M. \& Reiner, S. L. The lineage decisions of helper T cells. Nat. Rev. Immunol. 2, 933-944 (2002).

13. O'Shea, J. J. \& Paul, W. E. Mechanisms underlying lineage commitment and plasticity of helper CD4+ T cells. Science 327, 1098-102 (2010).

14. Hohl, T. M. Cell-Mediated Defense against Infection. in Mandell, Douglas, and Bennett's Principles and Practice of Infectious Diseases 50-69.e6 (Elsevier, 2015). doi:I0.1016/B978-I-4557-480 I-3.00006-0.

15. Szabo, S. J., Sullivan, B. M., Peng, S. L. \& Glimcher, L. H. Molecular mechanisms regulating Th I immune responses. Annu. Rev. Immunol. 2I, 7I3-758 (2003).

16. Spellberg, B. \& Edwards, J. E. Type I/Type 2 Immunity in Infectious Diseases. Clin. Infect. Dis. 32, 76-102 (2001).

17. Dendrou, C. A., Fugger, L. \& Friese, M. A. Immunopathology of multiple sclerosis. Nat. Rev. Immunol. 15, 545-558 (2015).

18. Basu, R., Hatton, R. D. \& Weaver, C. T. The ThI7 family: flexibility follows function. Immunol. Rev. 252, 89-103 (2013).

19. Froidure, A. et al. Asthma phenotypes and IgE responses. Eur. Respir. J. 47, 304-319 (2016).

20. Grigoriadis, N. \& van Pesch, V. A basic overview of multiple sclerosis immunopathology. Eur. J. Neurol. 22, 3-13 (2015). 
21. Kretschmer, K., Apostolou, I., Verginis, P. \& von Boehmer, H. Regulatory T Cells and AntigenSpecific Tolerance. in T Cell Regulation in Allergy, Asthma and Atopic Skin Diseases 8-I5 (KARGER, 2008). doi:10.II59/000I54846.

22. Vignali, D. A. A., Collison, L. W. \& Workman, C. J. How regulatory T cells work. Nat. Rev. Immunol. 8, 523-532 (2008).

23. Li, M. O. \& Flavell, R. A. Contextual Regulation of Inflammation: A Duet by Transforming Growth Factor- $\beta$ and Interleukin- I0. Immunity 28, 468-476 (2008).

24. Dejaco, C., Duftner, C., Grubeck-Loebenstein, B. \& Schirmer, M. Imbalance of regulatory T cells in human autoimmune diseases. Immunology II 7, 289-300 (2006).

25. Aranda-Anzaldo, A. The post-mitotic state in neurons correlates with a stable nuclear higher-order structure. Commun. Integr. Biol. 5, 134-139 (2012).

26. Bogie, J. F. J., Stinissen, P. \& Hendriks, J. J. A. Macrophage subsets and microglia in multiple sclerosis. Acta Neuropathol. 128, 19I-2I3 (20|4).

27. Hendriks, J. J. A., Teunissen, C. E., de Vries, H. E. \& Dijkstra, C. D. Macrophages and neurodegeneration. Brain Res. Brain Res. Rev. 48, I85-95 (2005).

28. Rosenberg, G. A. Matrix metalloproteinases in neuroinflammation. Glia 39, 279-29I (2002).

29. Fernández-Klett, F. \& Priller, J. The fibrotic scar in neurological disorders. Brain Pathol. 24, 404$4 \mid 3$ (20I4).

30. Krubitzer, L. \& Kahn, D. M. Nature versus nurture revisited: An old idea with a new twist. Prog. Neurobiol. 70, 33-52 (2003).

3I. Claassen, J. et al. Global cerebral edema after subarachnoid hemorrhage: Frequency, predictors, and impact on outcome. Stroke 33, 1225-1232 (2002).

32. Medawar, P. B. Immunity to homologous grafted skin; the fate of skin homografts. Br. J. Exp. Pathol. 29, 58-69 (1948).

33. J. Carson, M., M. Doose, J., Melchior, B., D. Schmid, C. \& C. Ploix, C. CNS immune privilege : hiding in plain sight. Immunol. Rev. 213, 48-65 (2009).

34. Louveau, A. et al. CNS lymphatic drainage and neuroinflammation are regulated by meningeal lymphatic vasculature. Nat. Neurosci. 21, 1380-1391 (2018).

35. Carson, M. J., Reilly, C. R., Sutcliffe, J. G. \& Lo, D. Mature microglia resemble immature antigenpresenting cells. Glia 22, 72-85 (1998).

36. Carson, M. J., Doose, J. M., Melchior, B., Schmid, C. D. \& Ploix, C. C. CNS immune privilege: Hiding in plain sight. Immunol. Rev. 213, 48-65 (2006).

37. Shechter, R., London, A. \& Schwartz, M. Orchestrated leukocyte recruitment to immune-privileged sites: Absolute barriers versus educational gates. Nat. Rev. Immunol. 13, 206-2I8 (2013).

38. Larochelle, C., Alvarez, J. I. \& Prat, A. How do immune cells overcome the blood-brain barrier in multiple sclerosis? FEBS Lett. 585, 3770-3780 (20I I).

39. Strominger, I. et al. The choroid plexus functions as a niche for T-cell stimulation within the central nervous system. Front. Immunol. 9, I-I5 (2018).

40. Smolders, J. et al. Tissue-resident memory T cells populate the human brain. Nat. Commun. 9, (2018).

4I. Walton, C. et al. Rising prevalence of multiple sclerosis worldwide: Insights from the Atlas of MS, third edition. Mult. Scler. J. 26, I8I6-I82I (2020). 
42. Segal, B. M. Stage-specific immune dysregulation in multiple sclerosis. J. Interf. Cytokine Res. 34, 633640 (20I4).

43. Noseworthy, J. H., Luccinetti, C., Rodriguez, M. \& Weinshenker, B. G. Multiple Sclerosis. N. Engl. J. Med. 343, 938-952 (2000).

44. Compston, A. \& Coles, A. Multiple sclerosis. Lancet 372, I502-15I7 (2008).

45. Baranzini, S. E. \& Oksenberg, J. R. The Genetics of Multiple Sclerosis: From 0 to 200 in 50 Years. Trends Genet. 33, 960-970 (2017).

46. Filippi, M. et al. Multiple sclerosis. Nat. Rev. Dis. Prim. 4, 43 (20।8).

47. Alcina, A. et al. Multiple sclerosis risk variant HLA-DRBI*I50I associates with high expression of DRBI gene in different human populations. PLoS One 7, 29819 (20I2).

48. Cotsapas, C. \& Mitrovic, M. Genome-wide association studies of multiple sclerosis. Clin. Transl. Immunol. 7, elol8 (2018).

49. Beecham, A. H. et al. Analysis of immune-related loci identifies 48 new susceptibility variants for multiple sclerosis. Nat. Genet. 45, I353-1362 (2013).

50. De Jager, P. L. et al. Meta-analysis of genome scans and replication identify CD6, IRF8 and TNFRSFIA as new multiple sclerosis susceptibility loci. Nat. Genet. 4I, 776-782 (2009).

5I. Emerging and Evolving Topics in Multiple Sclerosis Pathogenesis and Treatments. vol. 26 (Springer International Publishing, 20I5).

52. Miljković, D. \& Spasojević, I. Multiple Sclerosis: Molecular Mechanisms and Therapeutic Opportunities. Antioxid. Redox Signal. 19, 2286-2334 (20I3).

53. Hellings, $\mathrm{N}$. et al. T-cell reactivity to multiple myelin antigens in multiple sclerosis patients and healthy controls. J. Neurosci. Res. 63, 290-302 (2001).

54. Bielekova, B. et al. Expansion and Functional Relevance of High-Avidity Myelin-Specific CD4 + T Cells in Multiple Sclerosis. J. Immunol. 172, 3893-3904 (2004).

55. Compston, A. \& Coles, A. Multiple sclerosis. Lancet 372, I502-15I7 (2008).

56. Bhatia, R. \& Singh, N. Can We Treat Secondary Progressive Multiple Sclerosis Now? Ann. Indian Acad. Neurol. 22, I3|-136.

57. Multiple Sclerosis New Zealand. MS Treatments. https://www.msnz.org.nz/ms-treatments/ (202I).

58. Miller, D. H. et al. A Controlled Trial of Natalizumab for Relapsing Multiple Sclerosis. N. Engl. J. Med. 348, I5-23 (2003).

59. Steinman, L. Blocking adhesion molecules as therapy for multiple sclerosis: Natalizumab. Nat. Rev. Drug Discov. 4, 510-518 (2005).

60. Kelsey, R. (Nature R. The risk of natalizumab- associated PML is revealed. Nature Milestones S5 (20I8).

6I. Boster, A. L. et al. Lessons learned from fatal progressive multifocal leukoencephalopathy in a patient with multiple sclerosis treated with natalizumab. JAMA Neurol. 70, 398-402 (2013).

62. Clifford, D. B. et al. Natalizumab-associated progressive multifocal leukoencephalopathy in patients with multiple sclerosis: lessons from 28 cases. Lancet Neurol. 9, $438-446$ (2010).

63. Kappos, L. et al. Natalizumab treatment for multiple sclerosis: Updated recommendations for patient selection and monitoring. Lancet Neurol. 10, 745-758 (201 I). 
64. Mulero, P., Midaglia, L. \& Montalban, X. Ocrelizumab: a new milestone in multiple sclerosis therapy. Ther. Adv. Neurol. Disord. I I, I7562864I877302 (20I8).

65. Syed, Y. Y. Ocrelizumab: A Review in Multiple Sclerosis. CNS Drugs 32, 883-890 (2018).

66. Storch, M. K. et al. Multiple sclerosis: In situ evidence for antibody- and complement- mediated demyelination. Ann. Neurol. 43, 465-47I (1998).

67. Bigaut, K., De Seze, J. \& Collongues, N. Ocrelizumab for the treatment of multiple sclerosis. Expert Rev. Neurother. 19, 97-108 (2019).

68. Fyrst, H. \& Saba, J. D. An update on sphingosine-I-phosphate and other sphingolipid mediators. Nat. Chem. Biol. 6, 489-497 (20I0).

69. Chun, J., Kihara, Y., Jonnalagadda, D. \& Blaho, V. A. Fingolimod: Lessons learned and new opportunities for treating multiple sclerosis and other disorders. Annu. Rev. Pharmacol. Toxicol. 59, 149-170 (2019).

70. Hunter, S. F., Bowen, J. D. \& Reder, A. T. The Direct Effects of Fingolimod in the Central Nervous System: Implications for Relapsing Multiple Sclerosis. CNS Drugs 30, I35-147 (2016).

7I. Förster, R., Davalos-Misslitz, A. C. \& Rot, A. CCR7 and its ligands: Balancing immunity and tolerance. Nat. Rev. Immunol. 8, 362-37I (2008).

72. Schwab, S. R. \& Cyster, J. G. Finding a way out: Lymphocyte egress from lymphoid organs. Nat. Immunol. 8, I295-I30I (2007).

73. Chun, J., Kihara, Y., Jonnalagadda, D. \& Blaho, V. A. Fingolimod: Lessons Learned and New Opportunities for Treating Multiple Sclerosis and Other Disorders. Annu. Rev. Pharmacol. Toxicol. 59, I49-170 (2019).

74. Khatri, B. O. Fingolimod in the treatment of relapsing-remitting multiple sclerosis: Long-term experience and an update on the clinical evidence. Ther. Adv. Neurol. Disord. 9, I30-147 (2016).

75. Li, W., Xu, H. \& Testai, F. D. Mechanism of action and clinical potential of fingolimod for the treatment of stroke. Front. Neurol. 7, 26 (2016).

76. Medsafe. New Zealand Data Sheet Arava - leflunomid. https://www.medsafe.govt.nz/profs/Datasheet/a/Aravatab.pdf (2018).

77. Bar-Or, A., Pachner, A., Menguy-Vacheron, F., Kaplan, J. \& Wiendl, H. Teriflunomide and Its Mechanism of Action in Multiple Sclerosis. Drugs 74, 659-674 (2014).

78. Miller, A. E. Teriflunomide in multiple sclerosis: an update. Neurodegener. Dis. Manag. 7, 9-29 (2017).

79. Zeyda, M. et al. Disruption of the interaction of $T$ cells with antigen-presenting cells by the active leflunomide metabolite teriflunomide: Involvement of impaired integrin activation and immunologic synapse formation. Arthritis Rheum. 52, 2730-2739 (2005).

80. Li, L., Liu, J. \& Zhang, D. Teriflunomide treatment of human monocyte-derived dendritic cells in vitro does not impair their maturation or ability to induce allogeneic T-cell responses. Mult. Scler. J. 18, 279-508 (2012).

8I. Miller, A. E. Teriflunomide in multiple sclerosis: an update. Neurodegener. Dis. Manag. 7, 9-29 (2017).

82. Wiendl, $\mathrm{H}$. et al. Characterizing the Impact of Teriflunomide on Adaptive Immune Cell Subsets , Repertoire, and Function in Patients With Relapsing-Remitting MS. in 3 /st Congress of the European Committee for Treatment and Research in Multiple Sclerosis (20I5). 
83. Confavreux, C. et al. Oral teriflunomide for patients with relapsing multiple sclerosis (TOWER): A randomised, double-blind, placebo-controlled, phase 3 trial. Lancet Neurol. I3, 247-256 (2014).

84. O'Connor, P. et al. Randomized Trial of Oral Teriflunomide for Relapsing Multiple Sclerosis. New England Journal of Medicine vol. 365 (201 I).

85. O'Connor, P. et al. Randomized Trial of Oral Teriflunomide for Relapsing Multiple Sclerosis. N. Engl. J. Med. 365, I293-1303 (20II).

86. Bomprezzi, R. Dimethyl fumarate in the treatment of relapsing-remitting multiple sclerosis: an overview. Ther. Adv. Neurol. Disord. 8, 20-30 (2015).

87. Werdenberg, D., Joshi, R., Wolffram, S., Merkle, H. P. \& Langguth, P. Presystemic metabolism and intestinal absorption of antipsoriatic fumaric acid esters. Biopharm. Drug Dispos. 24, 259-273 (2003).

88. de Jong, R. et al. Selective stimulation of T helper 2 cytokine responses by the anti-psoriasis agent monomethylfumarate. Eur. J. Immunol. 26, 2067-2074 (1996).

89. Litjens, N. H. R. et al. Monomethylfumarate affects polarization of monocyte-derived dendritic cells resulting in down-regulated ThI lymphocyte responses. Eur. J. Immunol. 34, 565-575 (2004).

90. Moharregh-Khiabani, D., Linker, R., Gold, R. \& Stangel, M. Fumaric Acid and its Esters: An Emerging Treatment for Multiple Sclerosis. Curr. Neuropharmacol. 7, 60-64 (2009).

91. Linker, R. A. et al. Fumaric acid esters exert neuroprotective effects in neuroinflammation via activation of the Nrf2 antioxidant pathway. Brain 134, 678-692 (20II).

92. Gold, R. et al. Placebo-Controlled Phase 3 Study of Oral BG-12 for Relapsing Multiple Sclerosis. N. Engl. J. Med. 367, I098-II 07 (20I2).

93. Fox, R. J. et al. Placebo-Controlled Phase 3 Study of Oral BG-12 or Glatiramer in Multiple Sclerosis. N. Engl. J. Med. 367, I087-1097 (20I2).

94. K., N. et al. Cost-effectiveness of disease-modifying therapy for multiple sclerosis: A population-based evaluation. Value in Health http://ovidsp.ovid.com/ovidweb.cgi?T=JS\&PAGE=reference\&D=emed9\&NEWS=N\&AN=700I520 2 (2009).

95. Baxter, A. G. The origin and application of experimental autoimmune encephalomyelitis. Nat. Rev. Immunol. 7, 904-9/2 (2007).

96. Constantinescu, C. S., Farooqi, N., O'Brien, K. \& Gran, B. Experimental autoimmune encephalomyelitis (EAE) as a model for multiple sclerosis (MS). Br. J. Pharmacol. 164, I079-II06 (20II).

97. Robinson, A. P., Harp, C. T., Noronha, A. \& Miller, S. D. The experimental autoimmune encephalomyelitis (EAE) model of MS. in I73-I89 (20I4). doi: I0.1016/B978-0-444-5200 I-2.00008$\mathrm{X}$.

98. Miller, S. D. \& Karpus, W. J. Experimental Autoimmune Encephalomyelitis in the Mouse. Curr. Protoc. Immunol. 77, (2007).

99. Lopes Pinheiro, M. A. et al. Immune cell trafficking across the barriers of the central nervous system in multiple sclerosis and stroke. Biochim. Biophys. Acta - Mol. Basis Dis. 1862, 46I-47I (2016).

100. Mortazavi, M. M. et al. The choroid plexus: A comprehensive review of its history, anatomy, function, histology, embryology, and surgical considerations. Child's Nerv. Syst. 30, 205-2I4 (2014).

10I. Chiba, Y. et al. Glucose, Fructose, and Urate Transporters in the Choroid Plexus Epithelium. Int. J. Mol. Sci. 2I, 7230 (2020). 
102. Dias, M. C., Mapunda, J. A., Vladymyrov, M. \& Engelhardt, B. Structure and junctional complexes of endothelial, epithelial and glial brain barriers. Int. J. Mol. Sci. 20, (2019).

103. Jackson, A., Ph, D. \& Fr, C. R. Choroid Plexus. Am. Brain Tumor Assoc. 2, I55-160 (1992).

104. Engelhardt, B. \& Ransohoff, R. M. Capture, crawl, cross: The T cell code to breach the blood-brain barriers. Trends Immunol. 33, 579-589 (2012).

105. Kierdorf, K., Masuda, T., Jordão, M. J. C. \& Prinz, M. Macrophages at CNS interfaces: ontogeny and function in health and disease. Nat. Rev. Neurosci. 20, 547-562 (2019).

106. Williams, J. L., Holman, D. W. \& Klein, R. S. Chemokines in the balance: Maintenance of homeostasis and protection at CNS barriers. Front. Cell. Neurosci. 8, I-I2 (20I4).

107. Shechter, R. et al. Recruitment of Beneficial M2 Macrophages to Injured Spinal Cord Is Orchestrated by Remote Brain Choroid Plexus. Immunity 38, 555-569 (2013).

108. Tietz, S. \& Engelhardt, B. Brain barriers: Crosstalk between complex tight junctions and adherens junctions. J. Cell Biol. 209, 493-506 (2015).

109. Meeker, R. B., Williams, K., Killebrew, D. A. \& Hudson, L. C. Cell trafficking through the choroid plexus. Cell Adhes. Migr. 6, 390-396 (2012).

1 10. Baruch, K. \& Schwartz, M. CNS-specific T cells shape brain function via the choroid plexus. Brain. Behav. Immun. 34, II-I6 (20I3).

III. De Graaf, M. T. et al. Central memory CD4+ T cells dominate the normal cerebrospinal fluid. Cytom. Part B - Clin. Cytom. 80 B, 43-50 (20II).

112. Marques, F. et al. The choroid plexus in health and in disease: dialogues into and out of the brain. Neurobiol. Dis. 107, 32-40 (2017).

113. Martirosian, V., Julian, A. \& Neman, J. The Role of the Choroid Plexus in the Pathogenesis of Multiple Sclerosis. Choroid Plex. Cerebrospinal Fluid Emerg. Roles CNS Dev. Maintenance, Dis. Progress. I03-127 (2016) doi:10.1016/B978-0-12-801740-I.00007-X.

I 14. Engelhardt, B., Wolburg-Buchholz, K. \& Wolburg, H. Involvement of the Choroid Plexus in Central Nervous System Inflammation. Microsc. Res. Tech. 52, II 2-129 (200I).

115. Dixon, G. A. \& Pérez, C. A. Multiple Sclerosis and the Choroid Plexus: Emerging Concepts of Disease Immunopathophysiology. Pediatr. Neurol. 1 03, 65-75 (2020).

116. Jafarzadeh, A. et al. Vitamin D down-regulates the expression of some ThI7 cell-related cytokines, key inflammatory chemokines, and chemokine receptors in experimental autoimmune encephalomyelitis. Nutr. Neurosci. 22, 725-737 (2019).

117. Takeshita, Y. \& Ransohoff, R. M. Inflammatory cell trafficking across the blood-brain barrier: Chemokine regulation and in vitro models. Immunol. Rev. 248, 228-239 (20I2).

118. Wiatr, M. et al. Distinct migratory pattern of naive and effector $\mathrm{T}$ cells through the blood-CSF barrier following Echovirus 30 infection. J. Neuroinflammation 16, I-19 (2019).

119. Kunis, G. et al. IFN- $\gamma$-dependent activation of the brain's choroid plexus for CNS immune surveillance and repair. Brain 136, 3427-3440 (2013).

120. Vercellino, M. et al. Involvement of the choroid plexus in multiple sclerosis autoimmune inflammation: A neuropathological study. J. Neuroimmunol. 199, I33-I4I (2008).

12I. Steffen, B. J., Breier, G., Butcher, E. C., Schulz, M. \& Engelhardt, B. ICAM-I, VCAM-I, and MAdCAM-I are expressed on choroid plexus epithelium but not endothelium and mediate binding of lymphocytes in vitro. Am. J. Pathol. 148, 1819-38 (1996). 
122. Kleine, T. O. \& Benes, L. Immune surveillance of the human central nervous system (CNS): Different migration pathways of immune cells through the blood-brain barrier and bloodcerebrospinal fluid barrier in healthy persons. Cytom. Part A 69, I47-I5I (2006).

123. Reboldi, A. et al. C-C chemokine receptor 6-regulated entry of $\mathrm{TH}-\mathrm{I} 7$ cells into the CNS through the choroid plexus is required for the initiation of EAE. Nat. Immunol. 1 0, 5I4-523 (2009).

124. Kim, $\mathrm{H}$. et al. Choroid plexus changes on magnetic resonance imaging in multiple sclerosis and neuromyelitis optica spectrum disorder. J. Neurol. Sci. 4I 5, (2020).

125. Haas, J. et al. The Choroid Plexus Is Permissive for a Preactivated Antigen-Experienced Memory BCell Subset in Multiple Sclerosis. Front. Immunol. I I, I (202I).

126. Restorick, S. M. et al. CCR6+ Th cells in the cerebrospinal fluid of persons with multiple sclerosis are dominated by pathogenic non-classic ThI cells and GM-CSF-only-secreting Th cells. Brain. Behav. Immun. 64, 7I-79 (20I7).

127. Sarrazin, S., Lamanna, W. C. \& Esko, J. D. Heparan sulfate proteoglycans. Cold Spring Harb. Perspect. Biol. 3, 549-556 (201 I).

128. Heyman, B. \& Yang, Y. Mechanisms of heparanase inhibitors in cancer therapy. Exp. Hematol. 44, $1002-1012$ (2016).

129. Cohen, E., Atzmon, R., Vlodavsky, I. \& Ilan, N. Heparanase processing by lysosomal/endosomal protein preparation. FEBS Lett. 579, 2334-2338 (2005).

130. Takaoka, M. et al. Heparanase Expression Correlates with Invasion and Poor Prognosis in Gastric Cancers. Lab. Investig. 83, 613-622 (2003).

I3I. Baraz, L., Haupt, Y., Elkin, M., Peretz, T. \& Vlodavsky, I. Tumor suppressor p53 regulates heparanase gene expression. Oncogene 25, 3939-3947 (2006).

132. Abboud-Jarrous, G. et al. Site-directed Mutagenesis, Proteolytic Cleavage, and Activation of Human Proheparanase. J. Biol. Chem. 280, I3568-I 3575 (2005).

133. Changyaleket, B., Deliu, Z., Chignalia, A. Z. \& Feinstein, D. L. Heparanase: Potential roles in multiple sclerosis. J. Neuroimmunol. 310, 72-8I (20I7).

134. Goodall, K. J., Poon, I. K. H., Phipps, S. \& Hulett, M. D. Soluble Heparan Sulfate Fragments Generated by Heparanase Trigger the Release of Pro-Inflammatory Cytokines through TLR-4. PLoS One 9, el09596 (2014).

135. O'Callaghan, P., Zhang, X. \& Li, J. P. Heparan Sulfate Proteoglycans as Relays of Neuroinflammation. J. Histochem. Cytochem. 66, 305-319 (2018).

136. Naparstek, Y., Cohen, I. R., Fuks, Z. \& Vlodavsky, I. Activated T lymphocytes produce a matrixdegrading heparan sulphate endoglycosidase. Nature 3 I0, 24I-244 (1984).

137. Willenborg, D. O. \& Parish, C. R. Inhibition of Passive Allergic Encephalomyelitis by Sulfated Polysaccharides. Ann. N. Y. Acad. Sci. 540, 543-545 (1988).

138. Lider, O. et al. Suppression of experimental autoimmune diseases and prolongation of allograft survival by treatment of animals with low doses of heparins. J. Clin. Invest. 83, 752-756 (1989).

139. Lider, O. et al. Inhibition of $\mathrm{T}$ lymphocyte heparanase by heparin prevents $\mathrm{T}$ cell migration and $\mathrm{T}$ cell-mediated immunity. Eur. J. Immunol. 20, 493-499 (1990).

140. de Mestre, A. M., Staykova, M. A., Hornby, J. R., Willenborg, D. O. \& Hulett, M. D. Expression of the heparan sulfate-degrading enzyme heparanase is induced in infiltrating CD4 $+\mathrm{T}$ cells in experimental autoimmune encephalomyelitis and regulated at the level of transcription by early growth response gene. J. Leukoc. Biol. 82, I289-1300 (2007). 
14I. Sotnikov, I. et al. Enzymatically Quiescent Heparanase Augments T Cell Interactions with VCAMI and Extracellular Matrix Components under Versatile Dynamic Contexts. J. Immunol. 172, 5 I85$5193(2004)$.

142. Floris, S., van den Born, J., Van der Pol, S. M. A., Dijkstra, C. D. \& de Vries, H. E. Heparan Sulfate Proteoglycans Modulate Monocyte Migration across Cerebral Endothelium. J. Neuropathol. Exp. Neurol. 62, 780-790 (2003).

143. Hershkoviz, R., Mor, F., Miao, H. Q., Vlodavsky, I. \& Lider, O. Differential effects of polysulfated polysaccharide on experimental encephalomyelitis, proliferation of autoimmune $T$ cells, and inhibition of heparanase activity. J. Autoimmun. 8, 74I-750 (1995).

144. Koliesnik, I. O. et al. The Heparan Sulfate Mimetic PG545 Modulates T Cell Responses and Prevents Delayed-Type Hypersensitivity. Front. Immunol. I I, (2020).

145. Zubkova, O. V et al. Dendrimer Heparan Sulfate Glycomimetics: Potent Heparanase Inhibitors for Anticancer Therapy. ACS Chem. Biol. 13, 3236-3242 (20I8).

146. Dredge, K. et al. The PG500 series: novel heparan sulfate mimetics as potent angiogenesis and heparanase inhibitors for cancer therapy. Invest. New Drugs 28, 276-283 (20I0).

147. Pijuan, J. et al. In vitro cell migration, invasion, and adhesion assays: From cell imaging to data analysis. Front. Cell Dev. Biol. 7, 107 (2019).

148. Chen, H.-C. Boyden Chamber Assay. in Cell Migration 015-022 (Humana Press). doi:10.1385/I59259-860-9:015.

149. Monnot, A. D. \& Zheng, W. Culture of Choroid Plexus Epithelial Cells and In Vitro Model of Blood-CSF Barrier. in vol. 946 I3-29 (2012).

150. Martínez-Cáceres, E. M. et al. Expression of chemokine receptors in the different clinical forms of multiple sclerosis. Mult. Scler. 8, 390-395 (2002).

15I. Murugesan, N. et al. Active induction of experimental autoimmune encephalomyelitis by MOG 35-55 peptide immunization is associated with differential responses in separate compartments of the choroid plexus. Fluids and Barriers of the CNS vol. 9 http://www.fluidsbarrierscns.com/content/9/I/I5 (2012).

152. Robichon, K., Patel, V., Connor, B. \& La Flamme, A. C. Clozapine reduces infiltration into the CNS by targeting migration in experimental autoimmune encephalomyelitis. J. Neuroinflammation I7, 53 (2020).

153. Stanford, M. M. \& Issekutz, T. B. The relative activity of CXCR3 and CCR5 ligands in T lymphocyte migration: concordant and disparate activities in vitro and in vivo. J. Leukoc. Biol. 74, 79I-799 (2003).

154. An, G. et al. Effects of CCL5 on the biological behavior of breast cancer and the mechanisms of its interaction with tumor-associated macrophages. Oncol. Rep. 42, 2499-25I I (20I9).

155. Hwaiz, R., Rahman, M., Syk, I., Zhang, E. \& Thorlacius, H. Racl-dependent secretion of plateletderived CCL5 regulates neutrophil recruitment via activation of alveolar macrophages in septic lung injury. J. Leukoc. Biol. 97, 975-984 (2015).

156. Locati, M. et al. Analysis of the Gene Expression Profile Activated by the CC Chemokine Ligand 5/RANTES and by Lipopolysaccharide in Human Monocytes. J. Immunol. I68, 3557-3562 (2002).

157. Huang, R. et al. CCL5 derived from tumor-associated macrophages promotes prostate cancer stem cells and metastasis via activating $\beta$-catenin/STAT3 signaling. Cell Death Dis. I I, (2020).

158. Lun, M. P., Monuki, E. S. \& Lehtinen, M. K. Development and functions of the choroid plexuscerebrospinal fluid system. Nat. Rev. Neurosci. 16, 445-457 (2015).

159. Porcionatto, M. A. The extracellular matrix provides directional cues for neuronal migration during 
cerebellar development. Brazilian J. Med. Biol. Res. 39, 313-320 (2006).

160. Enjoji, M. et al. Establishment and characterization of choroid plexus carcinoma cell lines: Connection between choroid plexus and immune systems. Japanese J. Cancer Res. 87, 893-899 (1996).

16I. Mesquita, S. D. et al. The choroid plexus transcriptome reveals changes in type I and II interferon responses in a mouse model of Alzheimer's disease. Brain. Behav. Immun. 49, 280-292 (2015).

162. Marques, F. et al. Lipocalin 2 is present in the EAE brain and is modulated by natalizumab. Front. Cell. Neurosci. 6, (2012).

163. Dwyer, J. M. \& Johnson, C. The use of concanavalin A to study the immunoregulation of human T cells. Clin. Exp. Immunol. 46, 237-49 (1981).

164. Bemer, V. \& Truffa-Bachi, P. T cell activation by concanavalin A in the presence of cyclosporin A: immunosuppressor withdrawal induces NFATp translocation and interleukin-2 gene transcription. Eur. J. Immunol 26, I48I-I 488 (1996).

165. de Petris, S. Concanavalin A receptors, immunoglobulins, and theta antigen of the lymphocyte surface. Interactions with concanavalin A and with Cytoplasmic structures. J. Cell Biol. 65, 123-146 (1975).

166. Baruch, K. et al. CNS-specific immunity at the choroid plexus shifts toward destructive Th2 inflammation in brain aging. Proc. Natl. Acad. Sci. U. S. A. I I 0, 2264-2269 (20I3).

167. Vercellino, M. et al. Elevated levels of proinflammatory cytokines in cerebrospinal fluid of multiple sclerosis patients. Front. Cell. Neurosci. 9, I-10 (20I2).

168. Meeker, R. B., Williams, K., Killebrew, D. A. \& Hudson, L. C. Cell trafficking through the choroid plexus. Cell Adh. Migr. 6, 390-396 (2012).

169. Khaibullin, T. et al. Elevated levels of proinflammatory cytokines in cerebrospinal fluid of multiple sclerosis patients. Front. Immunol. 8, I-10 (2017).

170. Albini, A. Extracellular Matrix Invasion in Metastases and Angiogenesis: Commentary on the Matrigel "Chemoinvasion Assay". Cancer Res. 76, 4595-4597 (2016).

17I. Strazielle, N., Creidy, R., Malcus, C., Boucraut, J. \& Ghersi-Egea, J.-F. T-Lymphocytes Traffic into the Brain across the Blood-CSF Barrier: Evidence Using a Reconstituted Choroid Plexus Epithelium. PLoS One I I, e0I50945 (2016).

172. Lazarevic, I. \& Engelhardt, B. Modeling immune functions of the mouse blood-cerebrospinal fluid barrier in vitro: Primary rather than immortalized mouse choroid plexus epithelial cells are suited to study immune cell migration across this brain barrier. Fluids Barriers CNS I3, I-20 (2016).

173. Vogel, D. Y. S. et al. GM-CSF promotes migration of human monocytes across the blood brain barrier. Eur. J. Immunol. 45, I808-1819 (20I5).

174. Menheniott, T. R., Charalambous, M. \& Ward, A. Derivation of Primary Choroid Plexus Epithelial Cells from the Mouse. in vol. 633 207-220 (2010).

175. Srinivasan, B. et al. TEER measurement techniques for in vitro barrier model systems. J. Lab. Autom. 20, $107-26$ (2015).

176. Baehr, C., Reichel, V. \& Fricker, G. Choroid plexus epithelial monolayers - A cell culture model from porcine brain. Cerebrospinal Fluid Res. 3, I-I4 (2006).

177. Yao, L., Xue, X., Yu, P., Ni, Y. \& Chen, F. Evans Blue Dye: A Revisit of Its Applications in Biomedicine. Contrast Media Mol. Imaging 20 I8, I-10 (2018). 
178. Bílková, B. et al. Application of Concanavalin A during immune responsiveness skin-swelling tests facilitates measurement interpretation in mammalian ecology. Ecol. Evol. 6, 455I-4564 (2016).

179. Sallusto, F. et al. Switch in chemokine receptor expression upon TCR stimulation reveals novel homing potential for recently activated T cells. Eur. J. Immunol. 29, 2037-2045 (1999).

180. White, M. P. J. Innate immunomodulation with MIS416: mechanism of action in experimental autoimmune encephalomyelitis. (20I5).

18I. Pettinelli, C. B. \& McFarlin, D. E. Adoptive transfer of experimental allergic encephalomyelitis in $\mathrm{SJL} / \mathrm{J}$ mice after in vitro activation of lymph node cells by myelin basic protein: requirement for Lyt I+ 2- T lymphocytes. J. Immunol. 127, I420-3 (198I).

182. Bettelli, E. et al. Loss of T-bet, But Not STATI, Prevents the Development of Experimental Autoimmune Encephalomyelitis. J. Exp. Med. 200, 79-87 (2004).

183. Nath, N., Prasad, R., Giri, S., Singh, A. K. \& Singh, I. T-bet is essential for the progression of experimental autoimmune encephalomyelitis. Immunology I I 8, 384-39| (2006).

184. Panitch, H. S., Hirsch, R. L., Schindler, J. \& Johnson, K. P. Treatment of multiple sclerosis with gamma interferon: Exacerbations associated with activation of the immune system. Neurology 37, 1097-1097 (1987).

185. Skurkovich, S. et al. Randomized study of antibodies to IFN- $\gamma$ and TNF- $\alpha$ in secondary progressive multiple sclerosis. Mult. Scler. 7, 277-284 (200I).

186. Krakowski, M. \& Owens, T. Interferon- $\gamma$ confers resistance to experimental allergic encephalomyelitis. Eur. J. Immunol. 26, I64I-1646 (I996).

187. Sabatino, J. J., Shires, J., Altman, J. D., Ford, M. L. \& Evavold, B. D. Loss of IFN- $\gamma$ Enables the Expansion of Autoreactive CD4 + T Cells to Induce Experimental Autoimmune Encephalomyelitis by a Nonencephalitogenic Myelin Variant Antigen. J. Immunol. I 80, 445I-4457 (2008).

188. Willenborg, D. O., Fordham, S., Bernard, C. C., Cowden, W. B. \& Ramshaw, I. A. IFN-gamma plays a critical down-regulatory role in the induction and effector phase of myelin oligodendrocyte glycoprotein-induced autoimmune encephalomyelitis. J. Immunol. I 57, 3223-7 (1996).

189. Naves, R. et al. The Interdependent, Overlapping, and Differential Roles of Type I and II IFNs in the Pathogenesis of Experimental Autoimmune Encephalomyelitis. J. Immunol. I9I, 2967-2977 (20I3).

190. Xiao, B.-G., Ma, C.-G., Xu, L.-Y., Link, H. \& Lu, C.-Z. IL-I2/IFN- $/$ /NO axis plays critical role in development of ThI-mediated experimental autoimmune encephalomyelitis. Mol. Immunol. 45, II9|-II 96 (2008).

191. Billiau, A. et al. Enhancement of experimental allergic encephalomyelitis in mice by antibodies against IFN-gamma. J. Immunol. I40, I506-10 (1988).

192. VOORTHUIS, J. A. C. et al. Suppression of experimental allergic encephalomyelitis by intraventricular administration of interferon-gamma in Lewis rats. Clin. Exp. Immunol. 81, I83-188 (2008).

193. Arellano, G., Ottum, P. A., Reyes, L. I., Burgos, P. I. \& Naves, R. Stage-Specific Role of InterferonGamma in Experimental Autoimmune Encephalomyelitis and Multiple Sclerosis. Front. Immunol. 6, (20I5).

194. Martha, S. R. et al. Expression of Cytokines and Chemokines as Predictors of Stroke Outcomes in Acute Ischemic Stroke. Front. Neurol. 10, (2020).

195. Tyler, P. C., Guimond, S. E., Turnbull, J. E. \& Zubkova, O. V. Single-Entity Heparan Sulfate 
Glycomimetic Clusters for Therapeutic Applications. Angew. Chemie Int. Ed. 54, 27I8-2723 (2015).

196. Sckisel, G. D. et al. Differential phenotypes of memory CD4 and CD8 T cells in the spleen and peripheral tissues following immunostimulatory therapy. J. Immunother. Cancer 5, I-I I (2017).

197. Kang, Z. et al. Astrocyte-Restricted Ablation of Interleukin-17-Induced Act I-Mediated Signaling Ameliorates Autoimmune Encephalomyelitis. Immunity 32, 4I4-425 (20I0).

198. Tzartos, J. S. et al. Interleukin-17 Production in Central Nervous System-Infiltrating T Cells and Glial Cells Is Associated with Active Disease in Multiple Sclerosis. Am. J. Pathol. 172, I46-155 (2008).

199. Waisman, A., Hauptmann, J. \& Regen, T. The role of IL-I7 in CNS diseases. Acta Neuropathol. I29, 625-637 (2015).

200. Setiadi, A. F. et al. IL-I7A is associated with the breakdown of the blood-brain barrier in relapsingremitting multiple sclerosis. J. Neuroimmunol. 332, I47-154 (2019).

20I. Huppert, J. et al. Cellular mechanisms of IL-I7-induced blood-brain barrier disruption. FASEB J. 24, 1023-1034 (2010).

202. Ni, P. et al. IL-I7A contributes to perioperative neurocognitive disorders through blood-brain barrier disruption in aged mice. J. Neuroinflammation I5, 332 (2018).

203. Göbel, K., Ruck, T. \& Meuth, S. G. Cytokine signaling in multiple sclerosis: Lost in translation. Mult. Scler. J. 24, 432-439 (20I8).

204. Petkovic, F. \& Castellano, B. The role of interleukin-6 in central nervous system demyelination. Neural Regen. Res. I I, I 922 (2016).

205. Schneider, A. et al. In Active Relapsing-Remitting Multiple Sclerosis, Effector T Cell Resistance to Adaptive Tregs Involves IL-6-Mediated Signaling. Sci. Transl. Med. 5, I70ra I5-I70ra I5 (20I3).

206. No Title. doi:https://dx.doi.org//0.3390\%2Fbrainsci60200I5.

207. Krei, K., Fredrikson, S., Fontana, A. \& Link, H. Interleukin-6 is elevated in plasma in multiple sclerosis. J. Neuroimmunol. 31, I47-I53 (199I).

208. Pegoretti, V., Baron, W., Laman, J. D. \& Eisel, U. L. M. Selective Modulation of TNF-TNFRs Signaling: Insights for Multiple Sclerosis Treatment. Front. Immunol. 9, (2018).

209. Williams, S. K. et al. Antibody-Mediated Inhibition of TNFRI Attenuates Disease in a Mouse Model of Multiple Sclerosis. PLoS One 9, e901I7 (2014).

210. Maier, O., Fischer, R., Agresti, C. \& Pfizenmaier, K. TNF receptor 2 protects oligodendrocyte progenitor cells against oxidative stress. Biochem. Biophys. Res. Commun. 440, 336-34I (20I3).

21I. Fischer, R., Wajant, H., Kontermann, R., Pfizenmaier, K. \& Maier, O. Astrocyte-specific activation of TNFR2 promotes oligodendrocyte maturation by secretion of leukemia inhibitory factor. Glia 62, 272-283 (2014).

212. Marcucci, F., Klein, B., Altevogt, P., Landolfo, S. \& Kirchner, H. Concanavalin A-Induced Interferon Gamma Production by Murine Spleen Cells and T Cell Lines. Lack of Correlation with Lyt I, 2 Phenotype. Immunobiology 166, 219-227 (1984).

213. Lees, J. R. \& Cross, A. H. A little stress is good: IFN- $\gamma$, demyelination, and multiple sclerosis. J. Clin. Invest. I I 7, 297-299 (2007).

214. Youssef, S. et al. The HMG-CoA reductase inhibitor, atorvastatin, promotes a Th2 bias and reverses paralysis in central nervous system autoimmune disease. Nature 420, 78-84 (2002).

215. Xiao, B. G., Ma, C. G., Xu, L. Y., Link, H. \& Lu, C. Z. IL-I2/IFN- $/ / N O$ axis plays critical role in 
development of ThI-mediated experimental autoimmune encephalomyelitis. Mol. Immunol. 45, II9I-II 96 (2008).

216. Lin, $W$. et al. The integrated stress response prevents demyelination by protecting oligodendrocytes against immune-mediated damage. J. Clin. Invest. I I 7, 448-456 (2007).

217. Mitchell, K., Yang, H.-Y. T., Berk, J. D., Tran, J. H. \& ladarola, M. J. Monocyte chemoattractant protein-I in the choroid plexus: A potential link between vascular pro-inflammatory mediators and the CNS during peripheral tissue inflammation. Neuroscience I58, 885-895 (2009).

218. Baruch, K. et al. Aging-induced type I interferon response at the choroid plexus negatively affects brain function. Science (80-. ). 346, 89-93 (2014).

219. Booss, J., Esiri, M. M., Tourtellotte, W. W. \& Mason, D. Y. Immunohistological analysis of T lymphocyte subsets in the central nervous system in chronic progressive multiple sclerosis. J. Neurol. Sci. 62, 219-232 (1983).

220. Hauser, S. L. et al. Immunohistochemical analysis of the cellular infiltrate in multiple sclerosis lesions. Ann. Neurol. 19, 578-587 (1986).

221. Kim, T.-S. \& Shin, E.-C. The activation of bystander CD8+ T cells and their roles in viral infection. Exp. Mol. Med. 5 I, I-9 (20I9).

222. Rodríguez-Lorenzo, S. et al. Inflammation of the choroid plexus in progressive multiple sclerosis: accumulation of granulocytes and T cells. Acta Neuropathol. Commun. 8, 9 (2020).

223. Parish, C. R. et al. Treatment of central nervous system inflammation with inhibitors of basement membrane degradation. I, 104-113 (1998).

224. Li, H. et al. A4B7 Integrin Inhibitors: a Patent Review. Expert Opin. Ther. Pat. 28, 903-9I7 (20I8).

225. Schwerk, C., Tenenbaum, T., Kim, K. S. \& Schroten, H. The choroid plexus a multi-role player during infectious diseases of the CNS. Front. Cell. Neurosci. 9, I-I I (20I5).

226. Baruch, K. \& Schwartz, M. CNS-specific T cells shape brain function via the choroid plexus. Brain. Behav. Immun. 34, II-I6 (20I3).

227. Solár, P., Zamani, A., Kubíčková, L., Dubový, P. \& Joukal, M. Choroid plexus and the bloodcerebrospinal fluid barrier in disease. Fluids Barriers CNS 17, I-29 (2020).

228. Breuer, J. et al. Blockade of MCAM/CDI46 impedes CNS infiltration of T cells over the choroid plexus. J. Neuroinflammation I5, I-12 (2018).

229. Knieke, K. et al. CDI52 (CTLA-4) Determines CD4 T Cell Migration In Vitro and In Vivo. PLoS One 4, e5702 (2009).

230. Harris, N. et al. Mechanism of action and efficacy of RX-III, a thieno[2,3-c]pyridine derivative and small molecule inhibitor of protein interaction with glycosaminoglycans (SMIGs), in delayed-type hypersensitivity, TNBS-induced colitis and experimental autoimmune encephal. Inflamm. Res. 65, 285-294 (2016).

23I. Takeuchi, H. Midkine and multiple sclerosis. Br. J. Pharmacol. I71, 93 I-935 (2014).

232. Muramatsu, T. Structure and function of midkine as the basis of its pharmacological effects. Br. J. Pharmacol. I7 I, 8I4-826 (2014).

233. Siebert, J. R. \& Osterhout, D. J. The inhibitory effects of chondroitin sulfate proteoglycans on oligodendrocytes. J. Neurochem. I I9, I76-188 (201 I).

234. Pendleton, J. C. et al. Chondroitin sulfate proteoglycans inhibit oligodendrocyte myelination through PTPo. Exp. Neurol. 247, II3-I2I (20I3). 
235. Deng, Y.-P. et al. Chondroitin sulfate proteoglycans impede myelination by oligodendrocytes after perinatal white matter injury. Exp. Neurol. 269, 213-223 (2015).

236. Karus, M. et al. Regulation of oligodendrocyte precursor maintenance by chondroitin sulphate glycosaminoglycans. Glia 64, 270-286 (2016).

237. Keough, M. B. et al. An inhibitor of chondroitin sulfate proteoglycan synthesis promotes central nervous system remyelination. Nat. Commun. 7, II3 I2 (2016).

238. Bitan, M. et al. Heparanase upregulates Th2 cytokines, ameliorating experimental autoimmune encephalitis. Mol. Immunol. 47, I890-1898 (2010).

239. Irony-Tur-Sinai, M. et al. A synthetic heparin-mimicking polyanionic compound inhibits central nervous system inflammation. J. Neurol. Sci. 206, 49-57 (2003).

240. Chung, J.-S., Tamura, K., Cruz, P. D. \& Ariizumi, K. DC-HIL-Expressing Myelomonocytic Cells Are Critical Promoters of Melanoma Growth. J. Invest. Dermatol. I34, 2784-2794 (2014).

24I. Chung, J.-S., Dougherty, I., Cruz, P. D. \& Ariizumi, K. Syndecan-4 Mediates the Coinhibitory Function of DC-HIL on T Cell Activation. J. Immunol. I79, 5778-5784 (2007).

242. Waterman, M. et al. Heparanase upregulation by colonic epithelium in inflammatory bowel disease. Mod. Pathol. 20, 8-14 (2007).

243. van Horssen, J., Bö, L., Dijkstra, C. D. \& de Vries, H. E. Extensive extracellular matrix depositions in active multiple sclerosis lesions. Neurobiol. Dis. 24, 484-49I (2006).

244. Mahad, D. J., Howell, S. J. L. \& Woodroofe, M. N. Expression of chemokines in the CSF and correlation with clinical disease activity in patients with multiple sclerosis. J. Neurol. Neurosurg. Psychiatry 72, 498-502 (2002).

245. Sørensen, T. L. et al. Expression of specific chemokines and chemokine receptors in the central nervous system of multiple sclerosis patients. J. Clin. Invest. 103, 807-8I5 (1999).

246. $\mathrm{Xu}, \mathrm{H}$. et al. The modulatory effects of lipopolysaccharide-stimulated $\mathrm{B}$ cells on differential T-cell polarization. Immunology I 25, 218-228 (2008).

247. Schetters, S. T. T., Gomez-Nicola, D., Garcia-Vallejo, J. J. \& Van Kooyk, Y. Neuroinflammation: Microglia and T cells get ready to tango. Front. Immunol. 8, (20I8).

248. Kunis, G. et al. IFN- $\gamma$-dependent activation of the brain's choroid plexus for CNS immune surveillance and repair. Brain 136, 3427-3440 (2013).

249. Gutter-Kapon, L. et al. Heparanase is required for activation and function of macrophages. Proc. Natl. Acad. Sci. I I3, E7808-E78I7 (2016).

250. Ludwin, S. K. The pathogenesis of multiple sclerosis: Relating human pathology to experimental studies. Journal of Neuropathology and Experimental Neurology vol. 65 https://academic.oup.com/jnen/article/65/4/305/2645249 (2006).

25I. Macchi, M. et al. Mature oligodendrocytes bordering lesions limit demyelination and favor myelin repair via heparan sulfate production. Elife 9, I-26 (2020).

252. Huda, S. et al. Neuromyelitis optica spectrum disorders. Clin. Med. (Northfield. II). 19, 169-176 (2019). 